Titanocene(III)-Mediated 5-exo-trig Radical Cyclization: en route to Spirooxindole Based Tetrahydrofuran and Bicyclic Lactone

Jothi Lakshmi Nallasivam, Tushar Kanti Chakraborty*

Department of Organic Chemistry, Indian Institute of Science, Bengaluru 560012, India

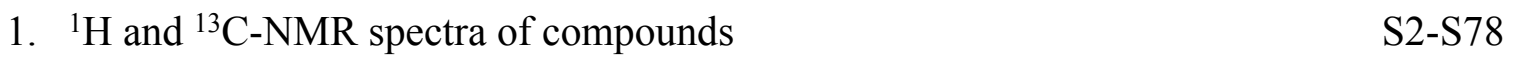

2. X-Ray crystal data 5a and 6c (CIF and thermal ellipsoid plot) S79-S82 
${ }^{1} \mathrm{H}-\mathrm{NMR}\left(\mathrm{CDCl}_{3}, 400 \mathrm{MHz}\right)$ and ${ }^{13} \mathrm{C}-\mathrm{NMR}\left(\mathrm{CDCl}_{3}, 100 \mathrm{MHz}\right)$ spectra of compound 3a
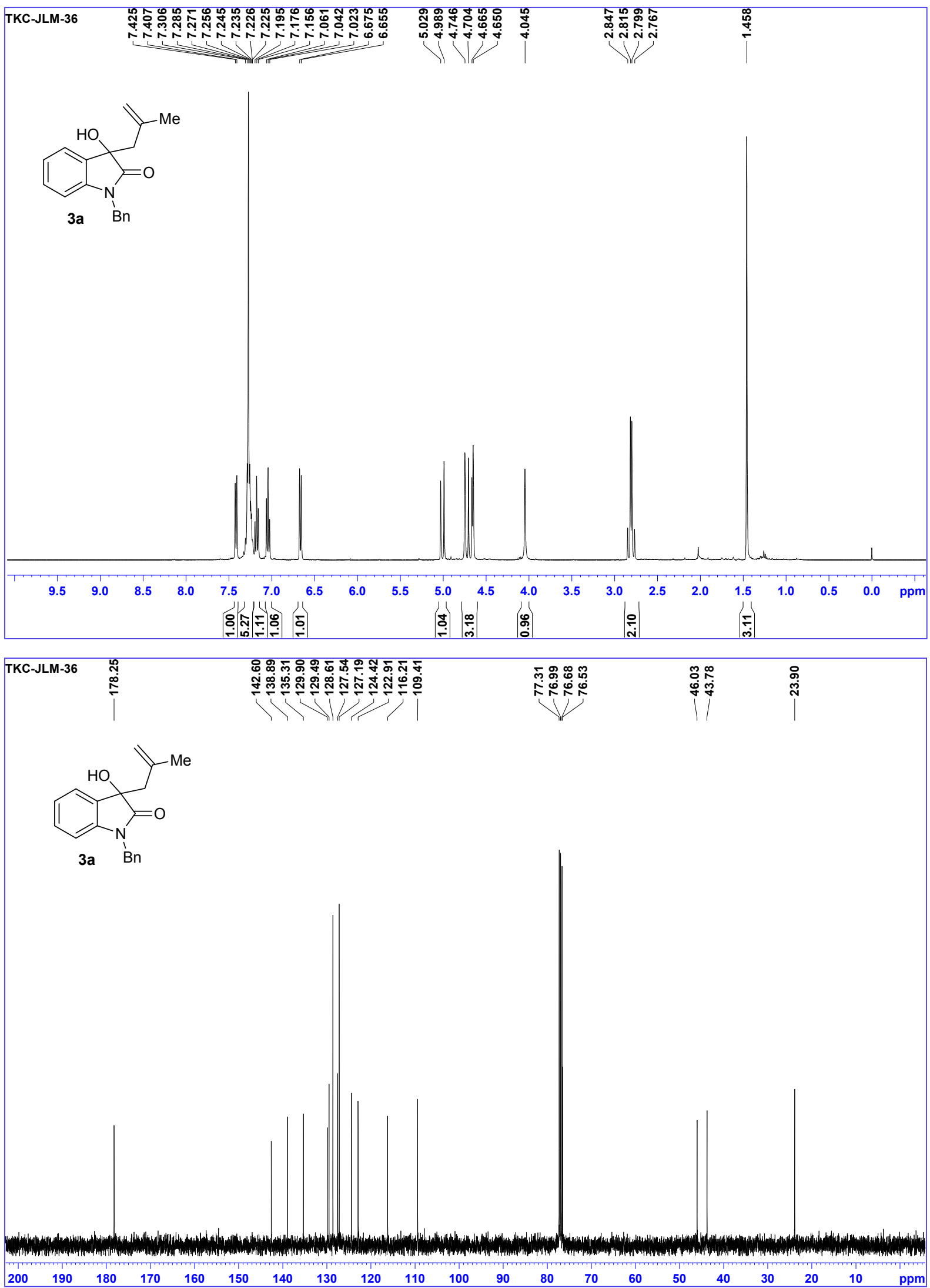
${ }^{1} \mathrm{H}-\mathrm{NMR}\left(\mathrm{CDCl}_{3}, 400 \mathrm{MHz}\right)$ and ${ }^{13} \mathrm{C}-\mathrm{NMR}\left(\mathrm{CDCl}_{3}, 100 \mathrm{MHz}\right)$ spectra of compound $\mathbf{3 b}$
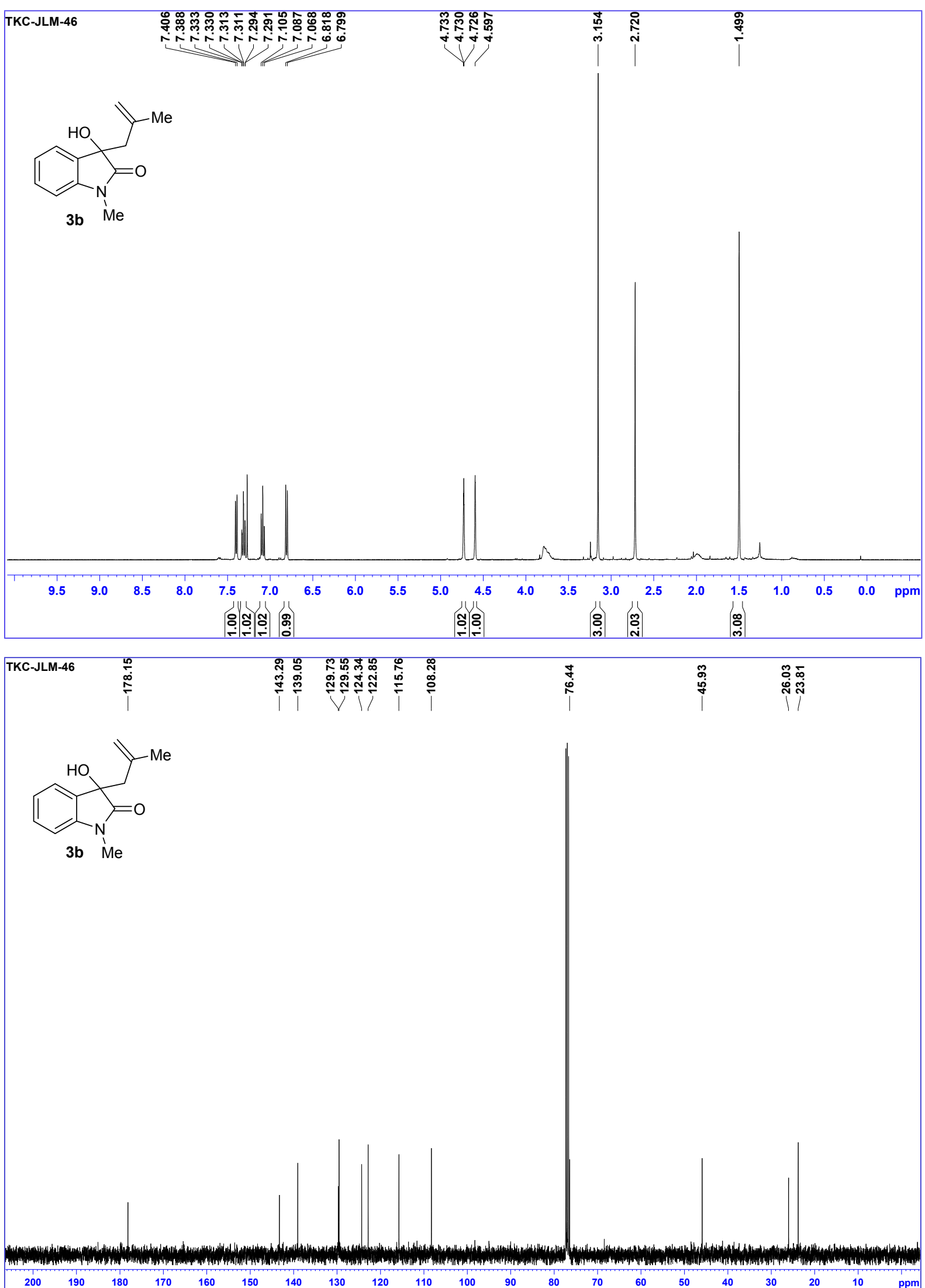
${ }^{1} \mathrm{H}-\mathrm{NMR}\left(\mathrm{CDCl}_{3}, 400 \mathrm{MHz}\right)$ and ${ }^{13} \mathrm{C}-\mathrm{NMR}\left(\mathrm{CDCl}_{3}, 100 \mathrm{MHz}\right)$ spectra of compound $3 \mathrm{c}$
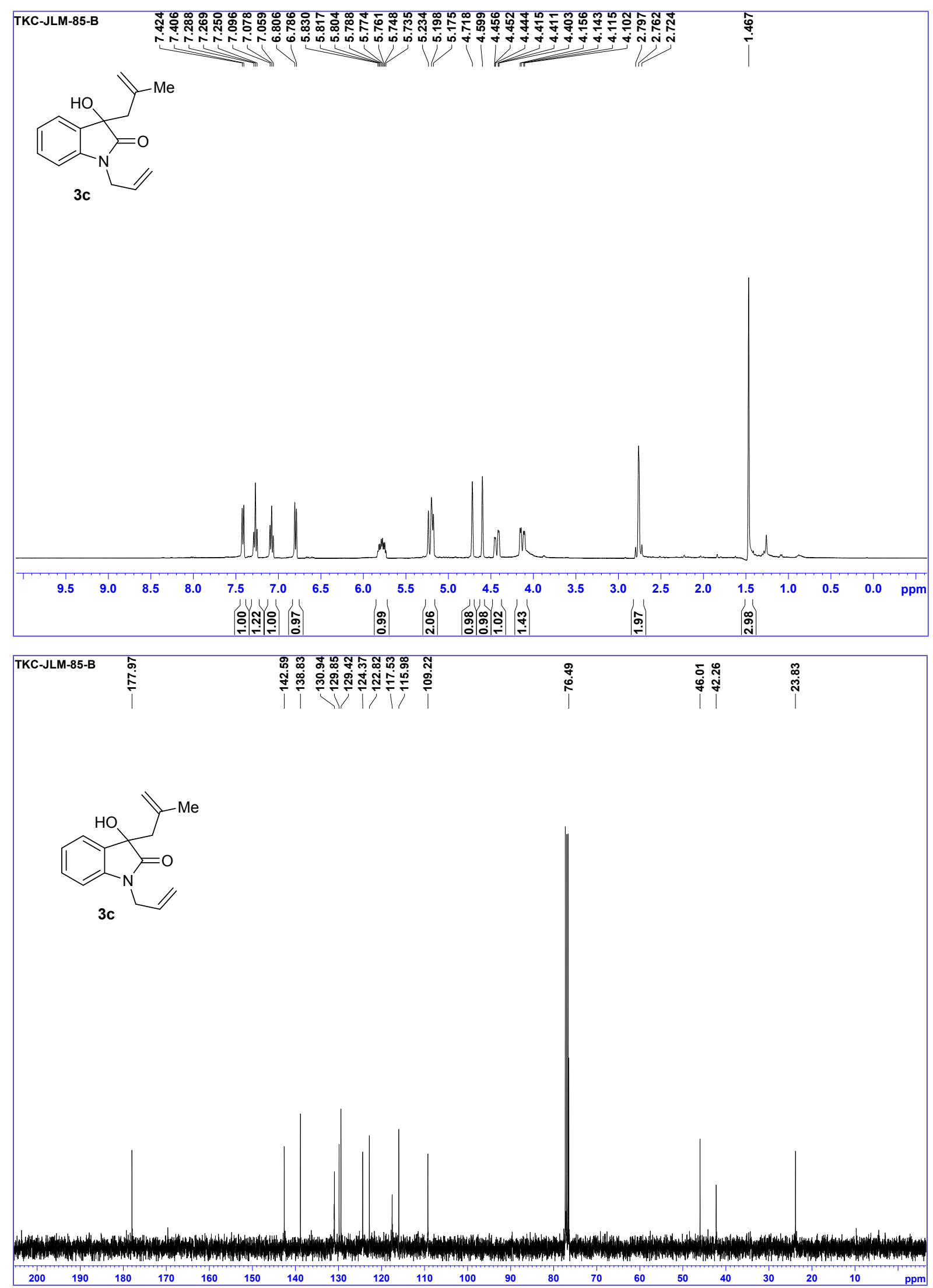
${ }^{1} \mathrm{H}-\mathrm{NMR}\left(\mathrm{CDCl}_{3}, 400 \mathrm{MHz}\right)$ and ${ }^{13} \mathrm{C}-\mathrm{NMR}\left(\mathrm{CDCl}_{3}, 100 \mathrm{MHz}\right)$ spectra of compound $\mathbf{3 d}$
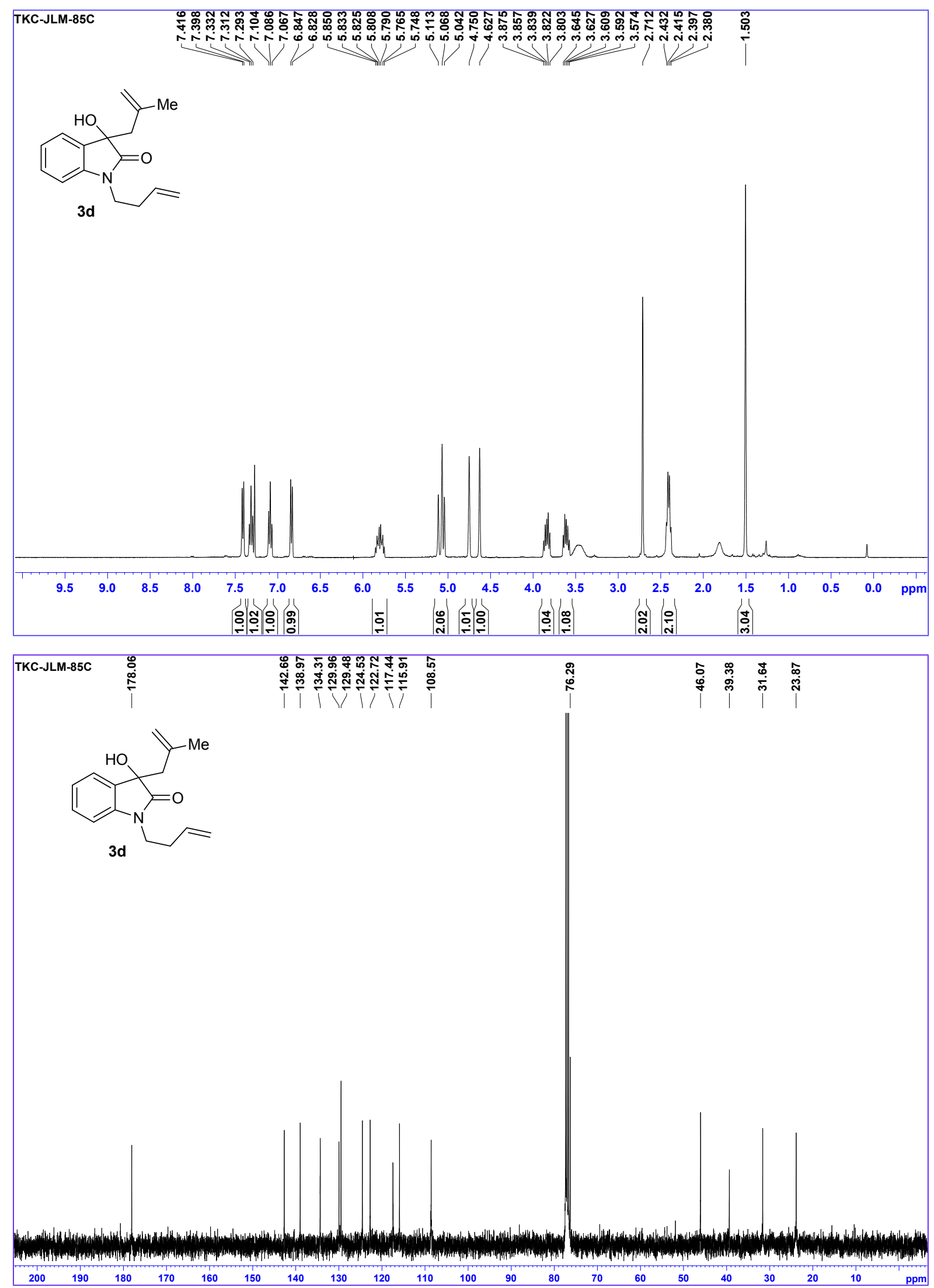
${ }^{1} \mathrm{H}-\mathrm{NMR}\left(\mathrm{CDCl}_{3}, 400 \mathrm{MHz}\right)$ and ${ }^{13} \mathrm{C}-\mathrm{NMR}\left(\mathrm{CDCl}_{3}, 100 \mathrm{MHz}\right)$ spectra of compound $\mathbf{3 e}$
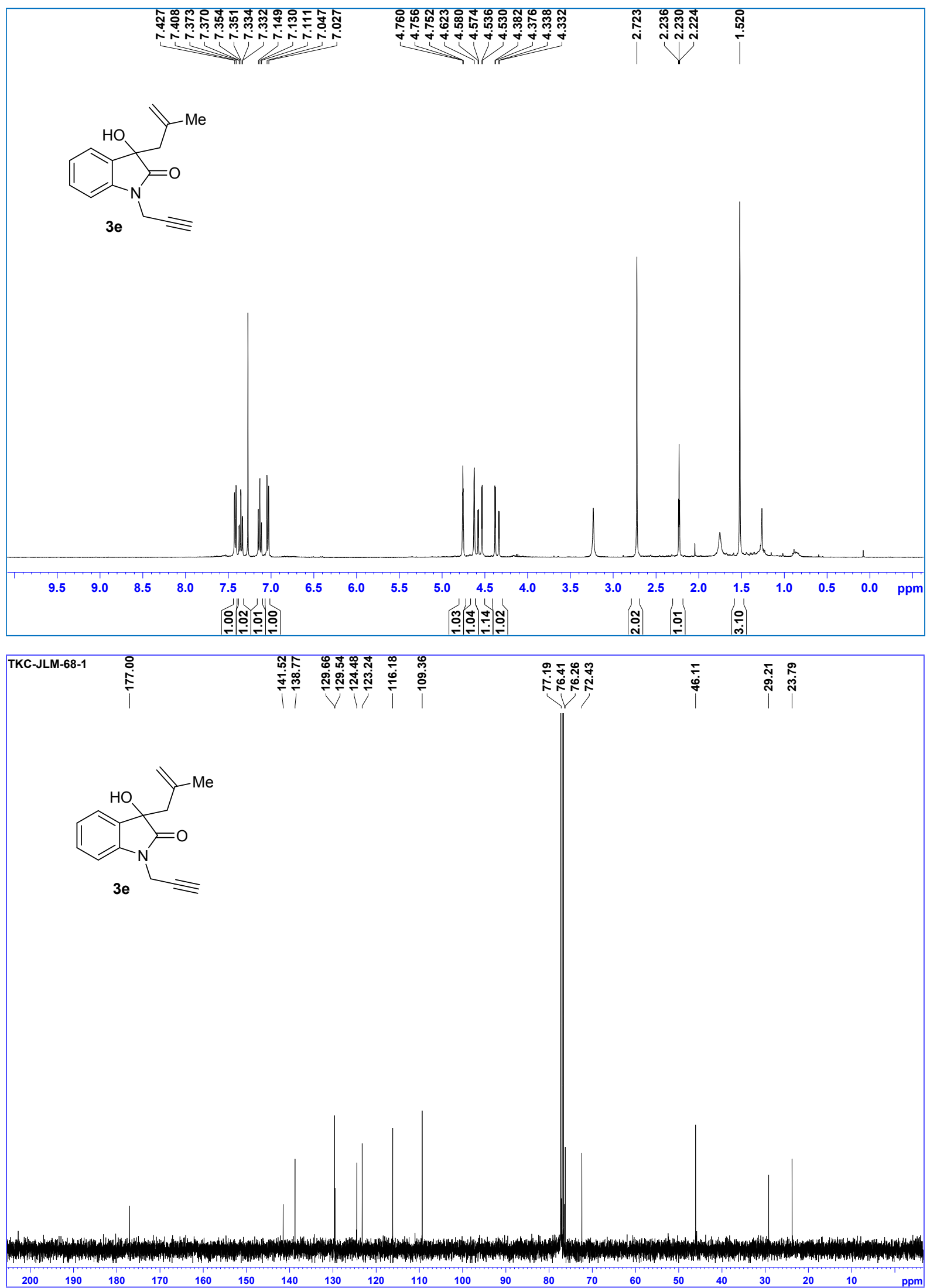
${ }^{1} \mathrm{H}-\mathrm{NMR}\left(\mathrm{CDCl}_{3}, 400 \mathrm{MHz}\right)$ and ${ }^{13} \mathrm{C}-\mathrm{NMR}\left(\mathrm{CDCl}_{3}, 100 \mathrm{MHz}\right)$ spectra of compound $\mathbf{3 f}$
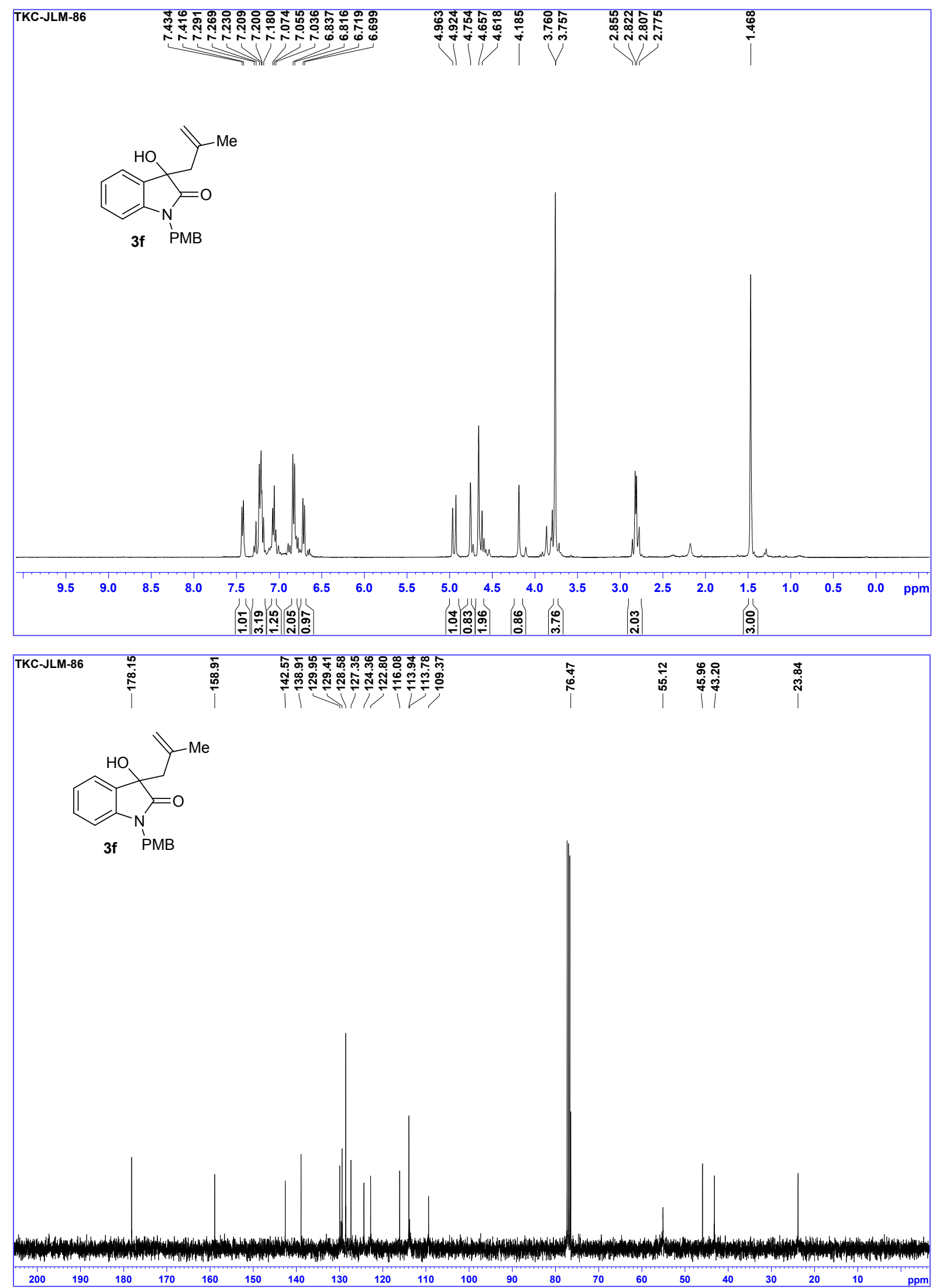
${ }^{1} \mathrm{H}-\mathrm{NMR}\left(\mathrm{CDCl}_{3}, 400 \mathrm{MHz}\right)$ and ${ }^{13} \mathrm{C}-\mathrm{NMR}\left(\mathrm{CDCl}_{3}, 100 \mathrm{MHz}\right)$ spectra of compound $\mathbf{3 g}$
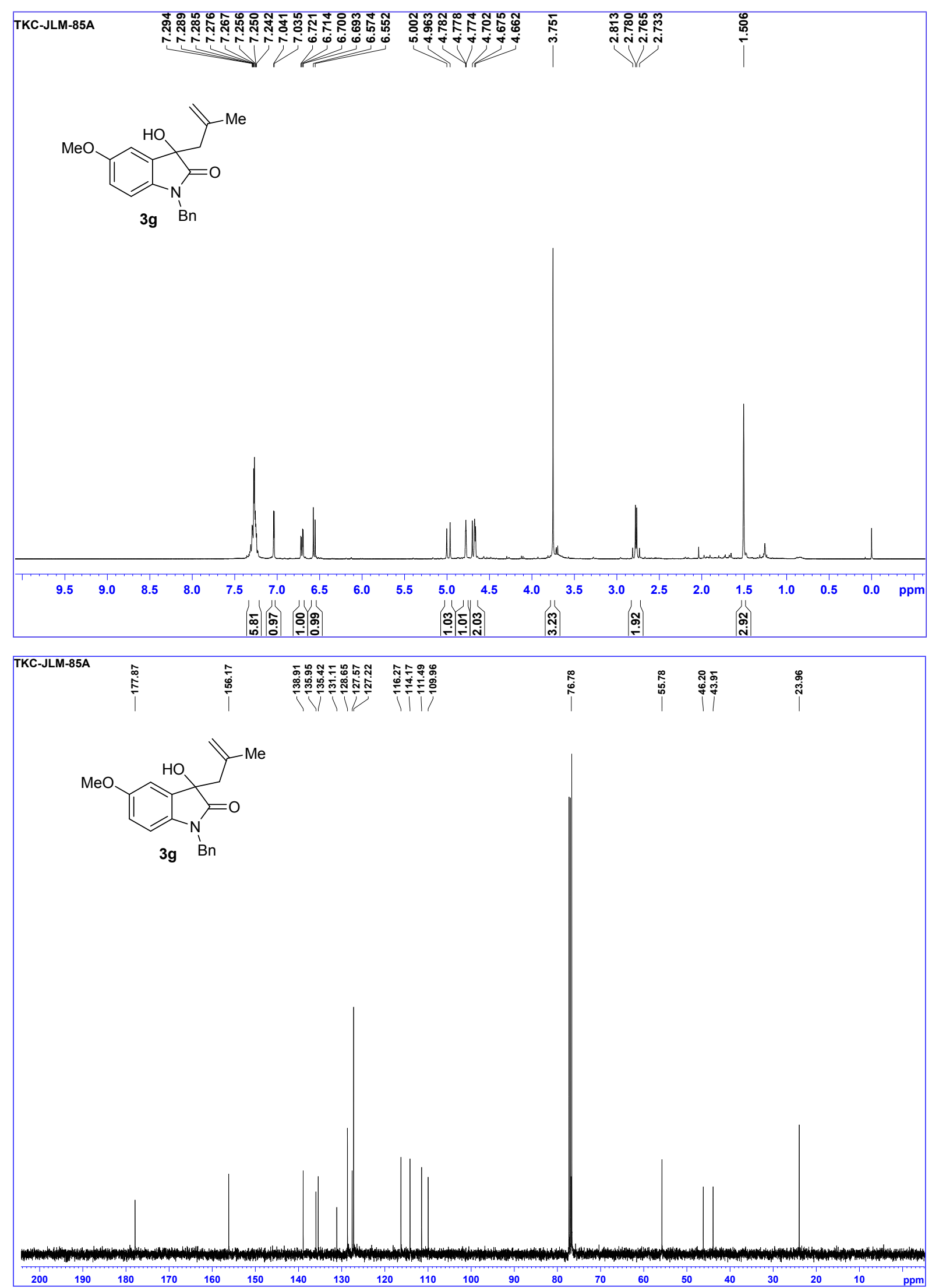
${ }^{1} \mathrm{H}-\mathrm{NMR}\left(\mathrm{CDCl}_{3}, 400 \mathrm{MHz}\right)$ and ${ }^{13} \mathrm{C}-\mathrm{NMR}\left(\mathrm{CDCl}_{3}, 100 \mathrm{MHz}\right)$ spectra of compound $\mathbf{3 h}$
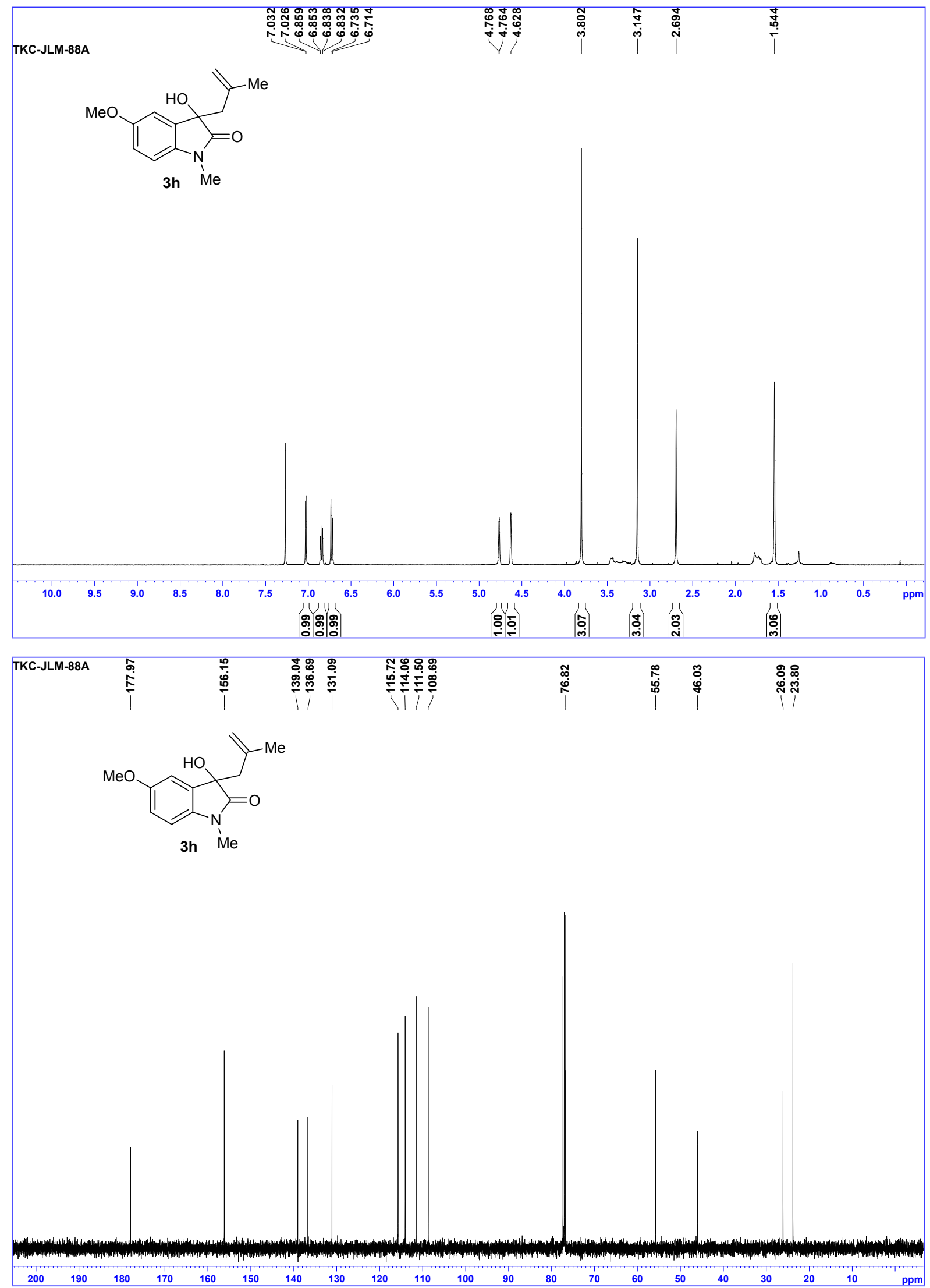
${ }^{1} \mathrm{H}-\mathrm{NMR}\left(\mathrm{CDCl}_{3}, 400 \mathrm{MHz}\right)$ and ${ }^{13} \mathrm{C}-\mathrm{NMR}\left(\mathrm{CDCl}_{3}, 100 \mathrm{MHz}\right)$ spectra of compound $3 \mathbf{i}$
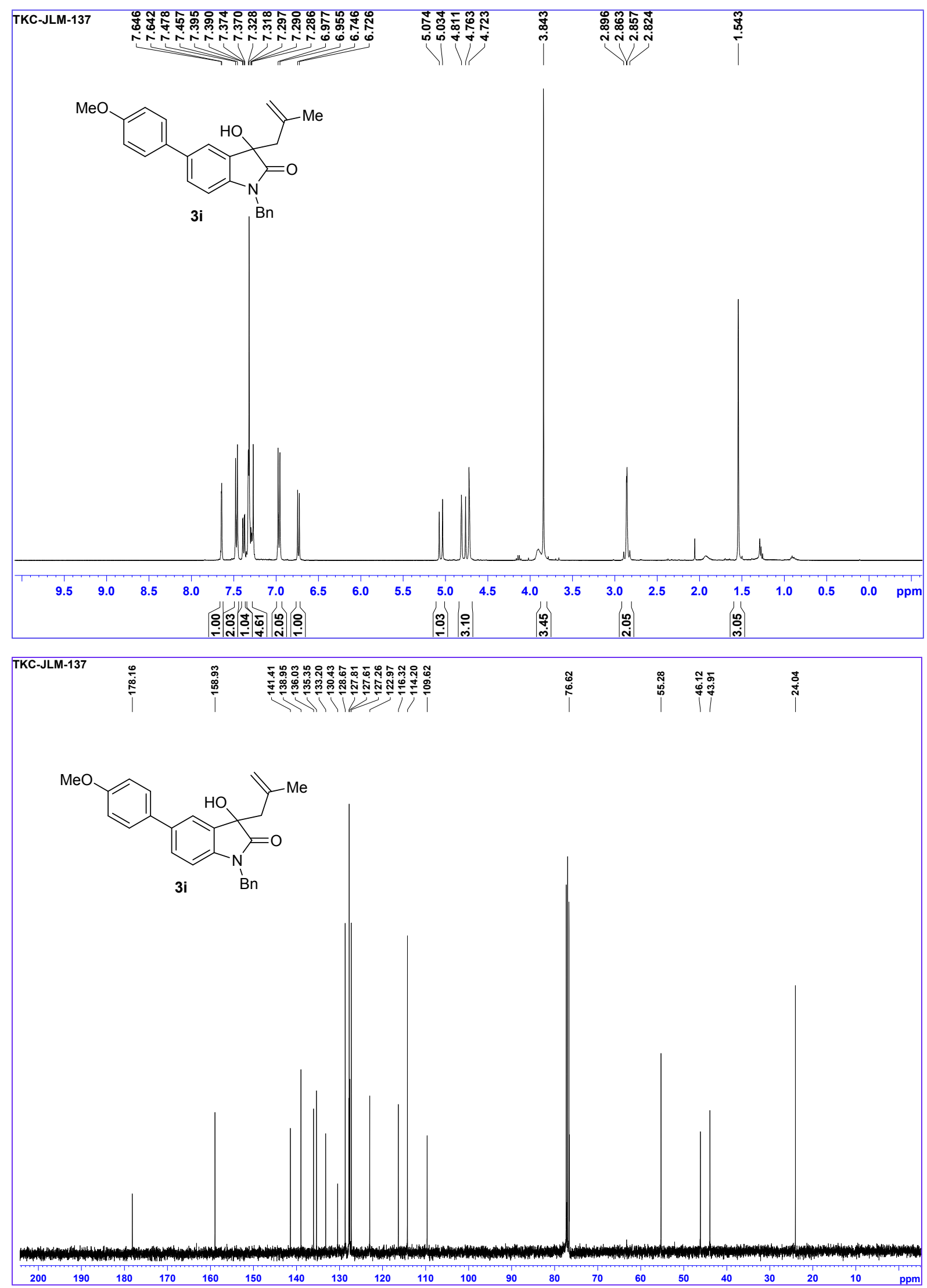
${ }^{1} \mathrm{H}-\mathrm{NMR}\left(\mathrm{CDCl}_{3}, 400 \mathrm{MHz}\right)$ and ${ }^{13} \mathrm{C}-\mathrm{NMR}\left(\mathrm{CDCl}_{3}, 100 \mathrm{MHz}\right)$ spectra of compound $\mathbf{3 j}$
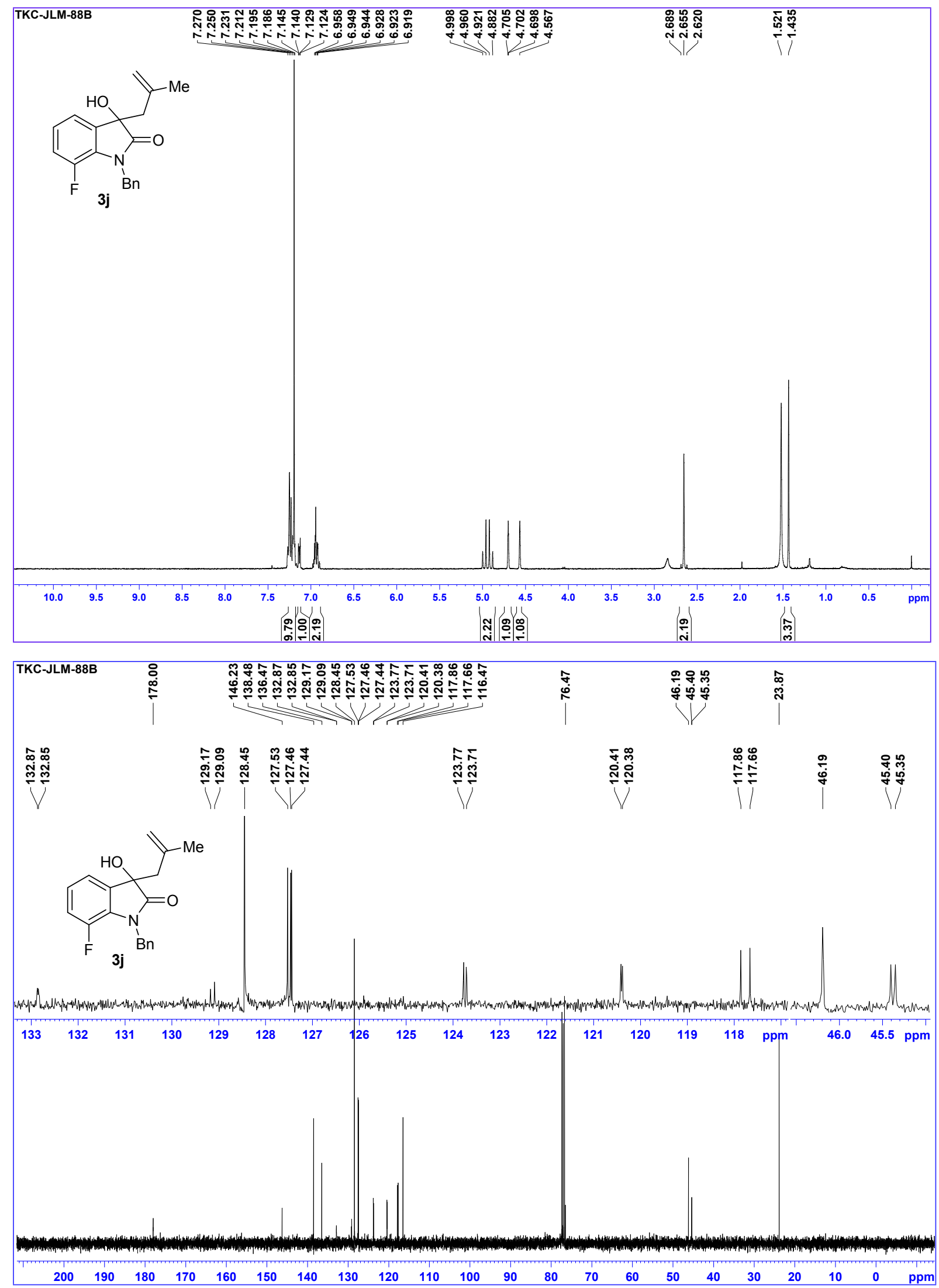
${ }^{1} \mathrm{H}-\mathrm{NMR}\left(\mathrm{CDCl}_{3}, 400 \mathrm{MHz}\right)$ and ${ }^{13} \mathrm{C}-\mathrm{NMR}\left(\mathrm{CDCl}_{3}, 100 \mathrm{MHz}\right)$ spectra of compound $4 \mathbf{a}$
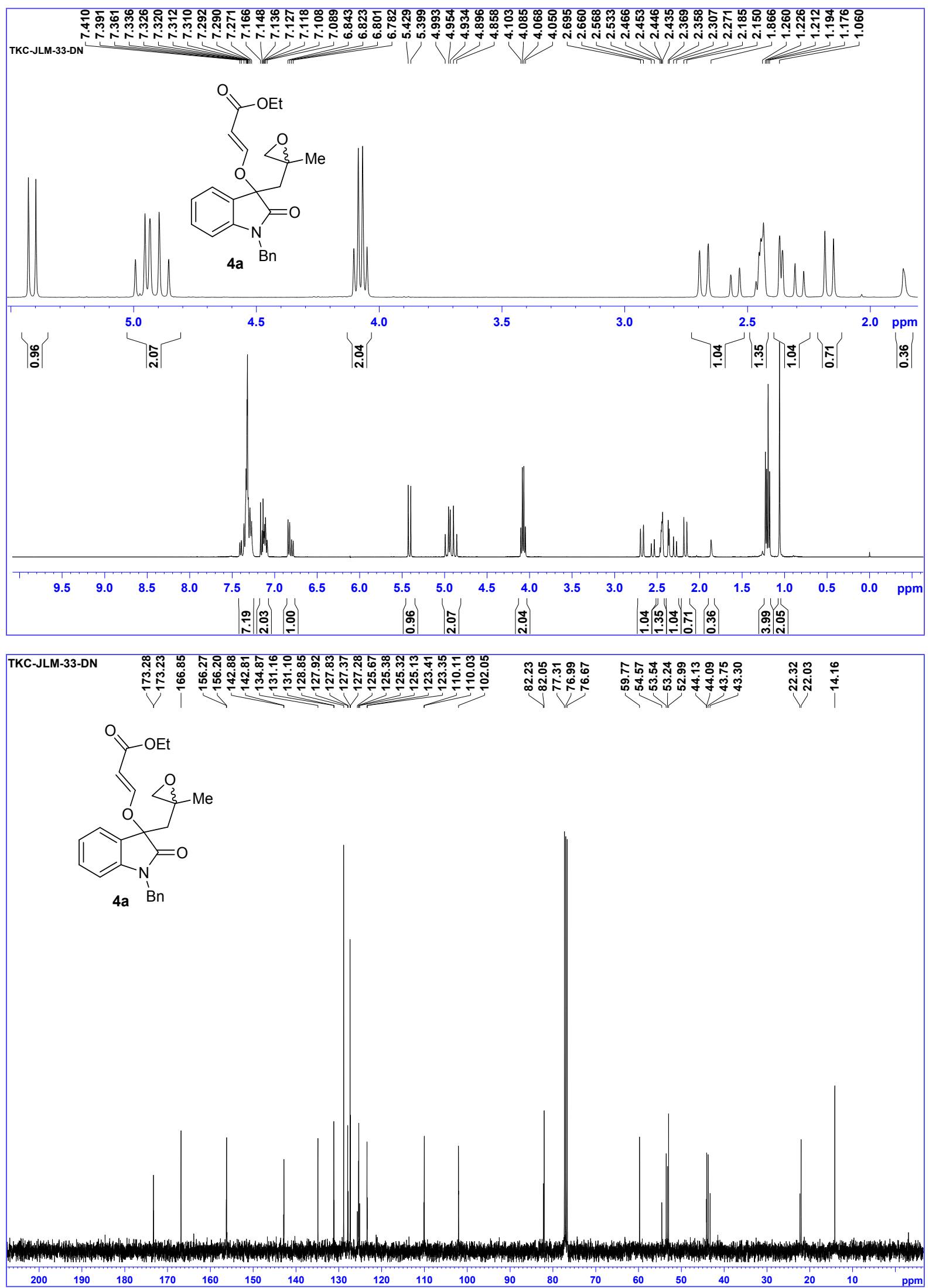
${ }^{1} \mathrm{H}-\mathrm{NMR}\left(\mathrm{CDCl}_{3}, 400 \mathrm{MHz}\right)$ and ${ }^{13} \mathrm{C}-\mathrm{NMR}\left(\mathrm{CDCl}_{3}, 100 \mathrm{MHz}\right)$ spectra of compound $\mathbf{4 b}$

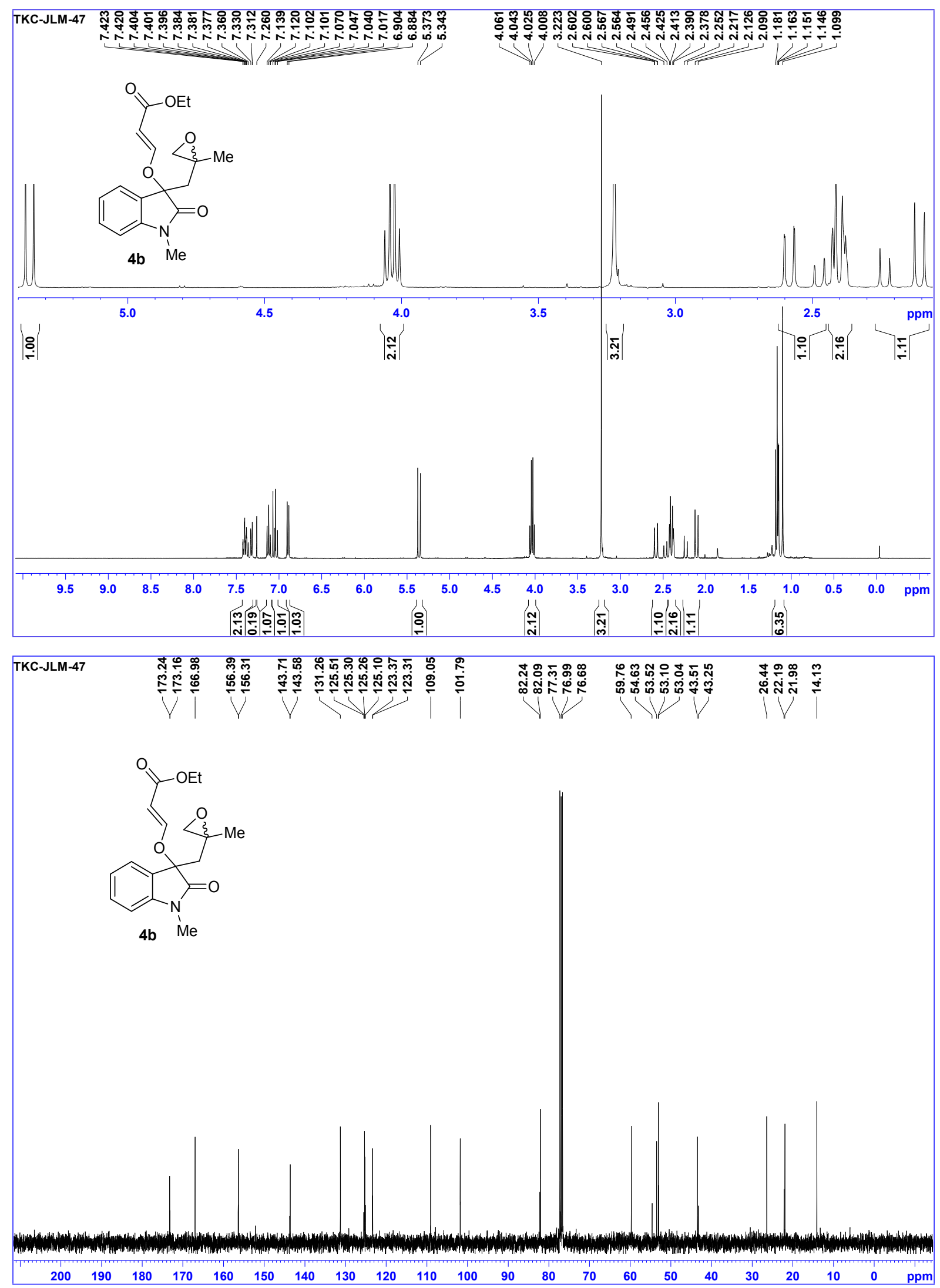


${ }^{1} \mathrm{H}-\mathrm{NMR}\left(\mathrm{CDCl}_{3}, 400 \mathrm{MHz}\right)$ and ${ }^{13} \mathrm{C}-\mathrm{NMR}\left(\mathrm{CDCl}_{3}, 100 \mathrm{MHz}\right)$ spectra of compound $\mathbf{4 c}$
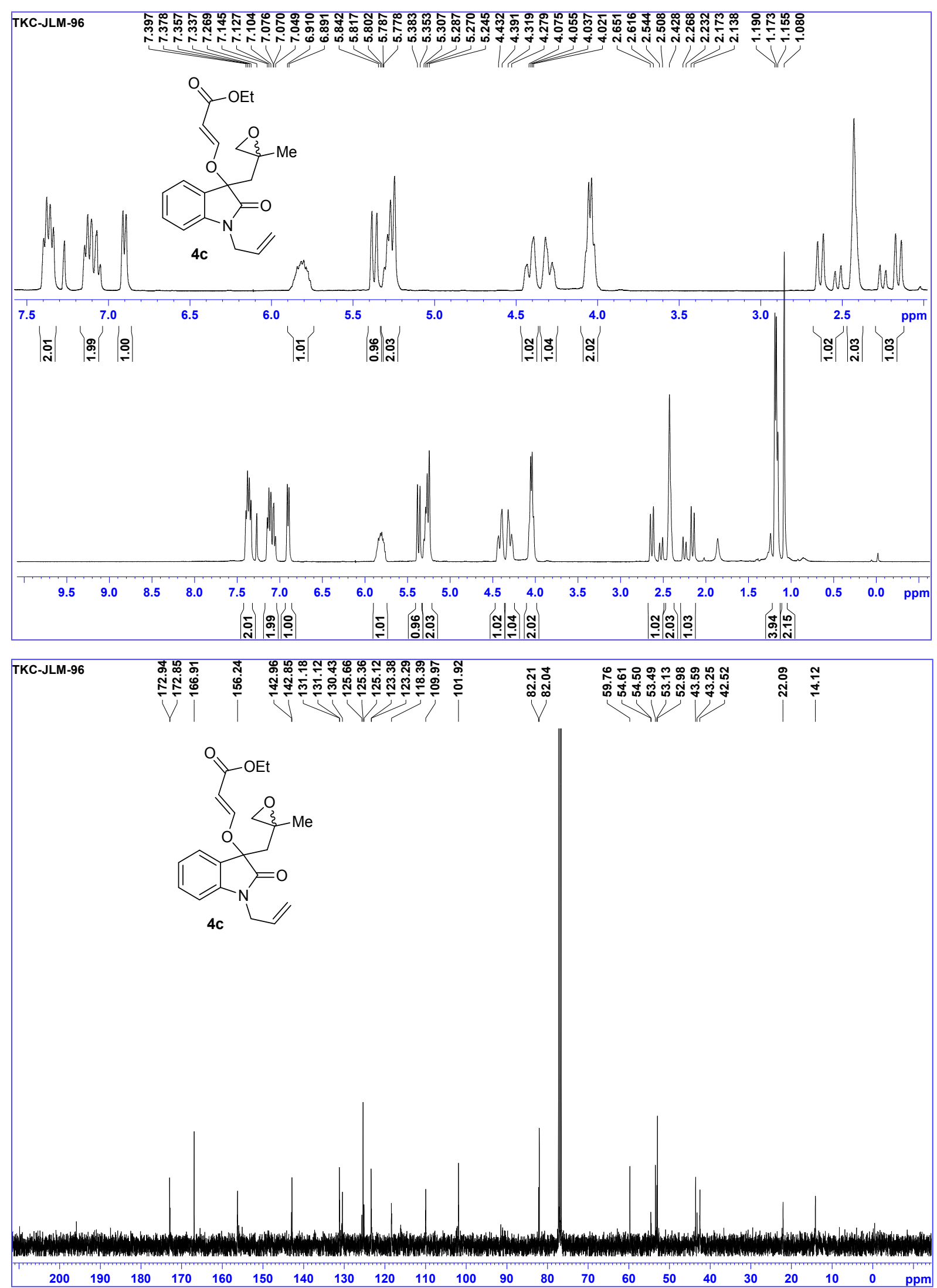
${ }^{1} \mathrm{H}-\mathrm{NMR}\left(\mathrm{CDCl}_{3}, 400 \mathrm{MHz}\right)$ and ${ }^{13} \mathrm{C}-\mathrm{NMR}\left(\mathrm{CDCl}_{3}, 100 \mathrm{MHz}\right)$ spectra of compound $4 \mathbf{d}$
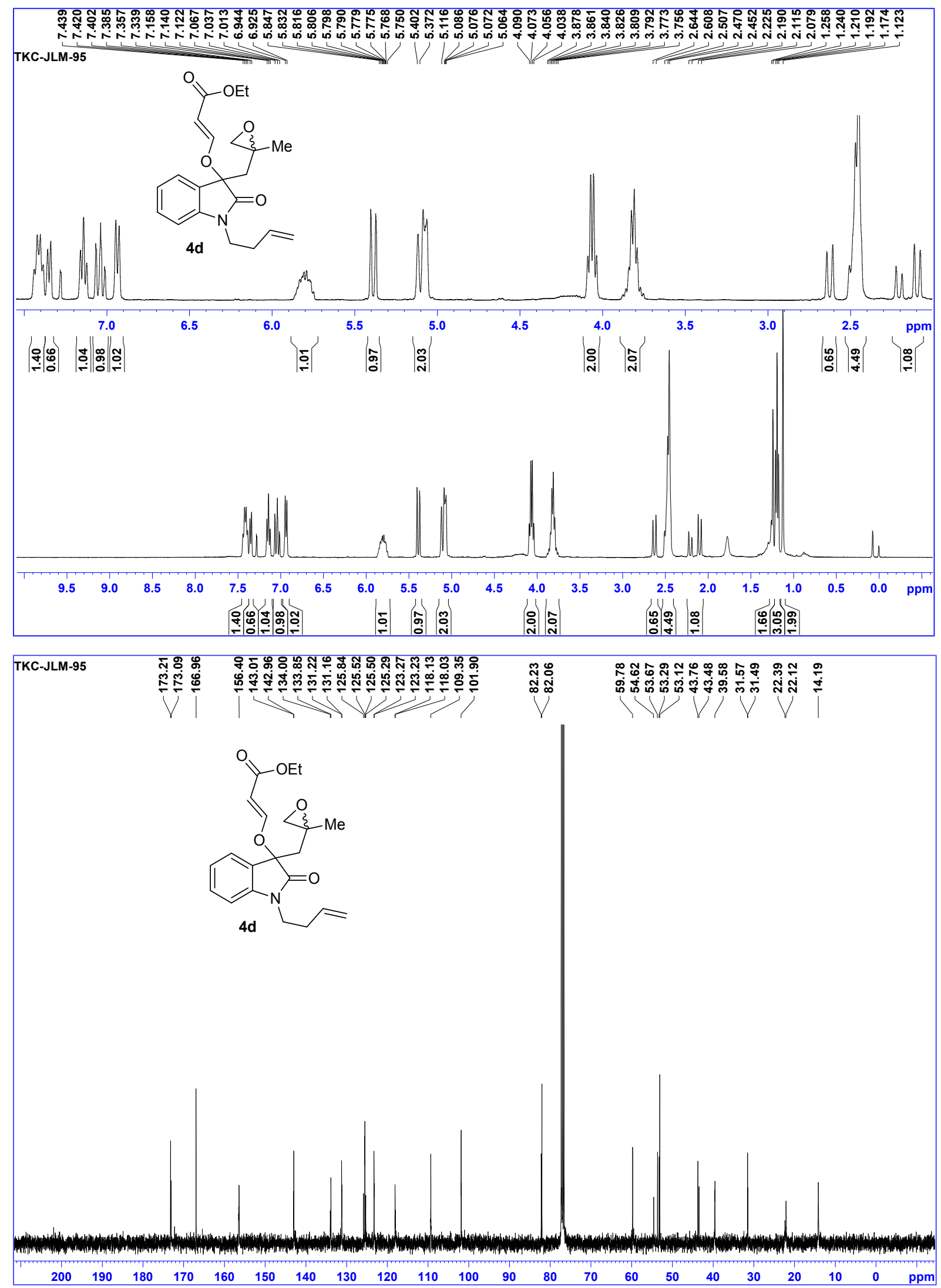
${ }^{1} \mathrm{H}-\mathrm{NMR}\left(\mathrm{CDCl}_{3}, 400 \mathrm{MHz}\right)$ and ${ }^{13} \mathrm{C}-\mathrm{NMR}\left(\mathrm{CDCl}_{3}, 100 \mathrm{MHz}\right)$ spectra of compound $4 \mathbf{e}$
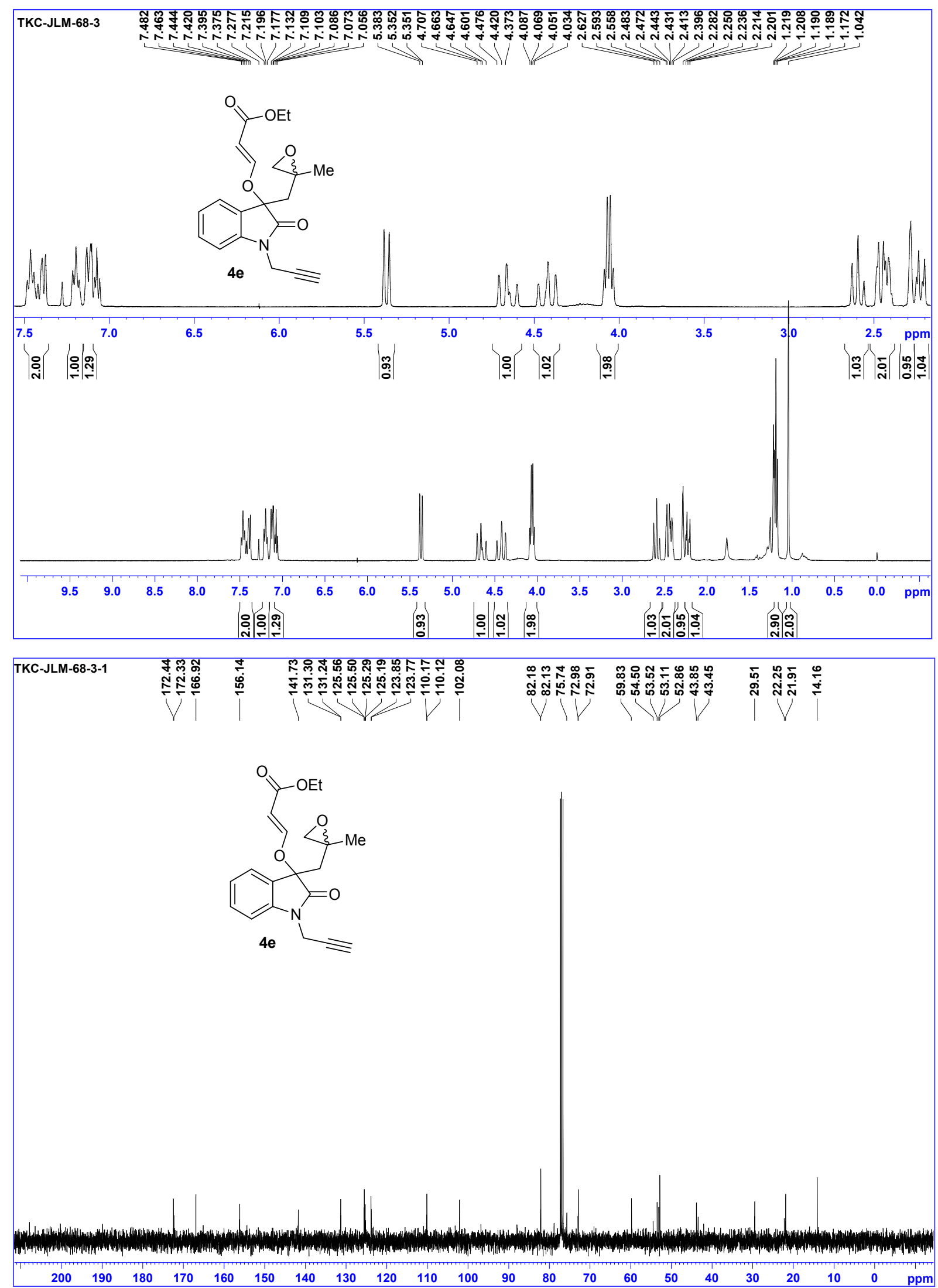
${ }^{1} \mathrm{H}-\mathrm{NMR}\left(\mathrm{CDCl}_{3}, 400 \mathrm{MHz}\right)$ and ${ }^{13} \mathrm{C}-\mathrm{NMR}\left(\mathrm{CDCl}_{3}, 100 \mathrm{MHz}\right)$ spectra of compound $\mathbf{4 f}$
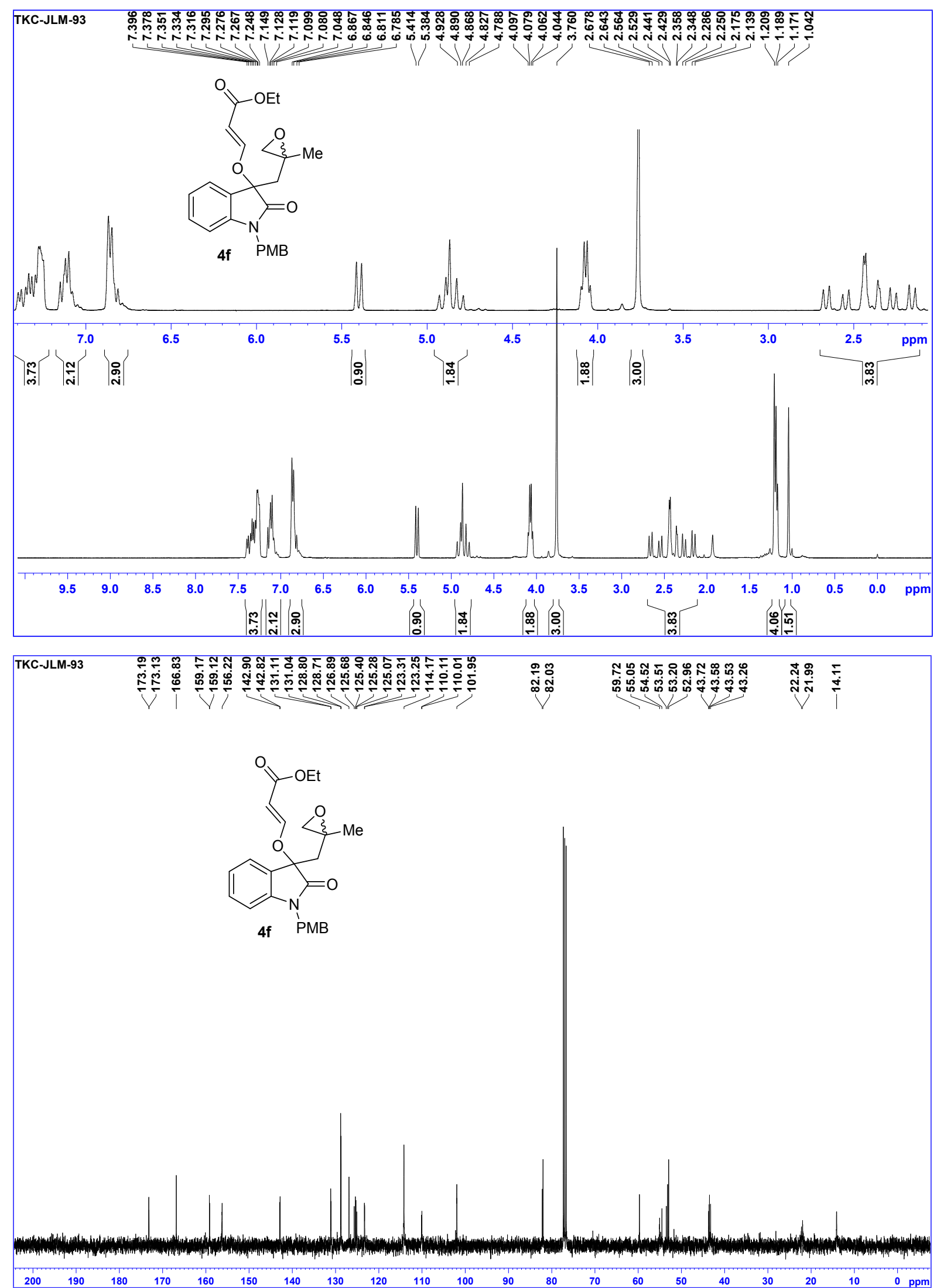
${ }^{1} \mathrm{H}-\mathrm{NMR}\left(\mathrm{CDCl}_{3}, 400 \mathrm{MHz}\right)$ and ${ }^{13} \mathrm{C}-\mathrm{NMR}\left(\mathrm{CDCl}_{3}, 100 \mathrm{MHz}\right)$ spectra of compound $\mathbf{4 g}$
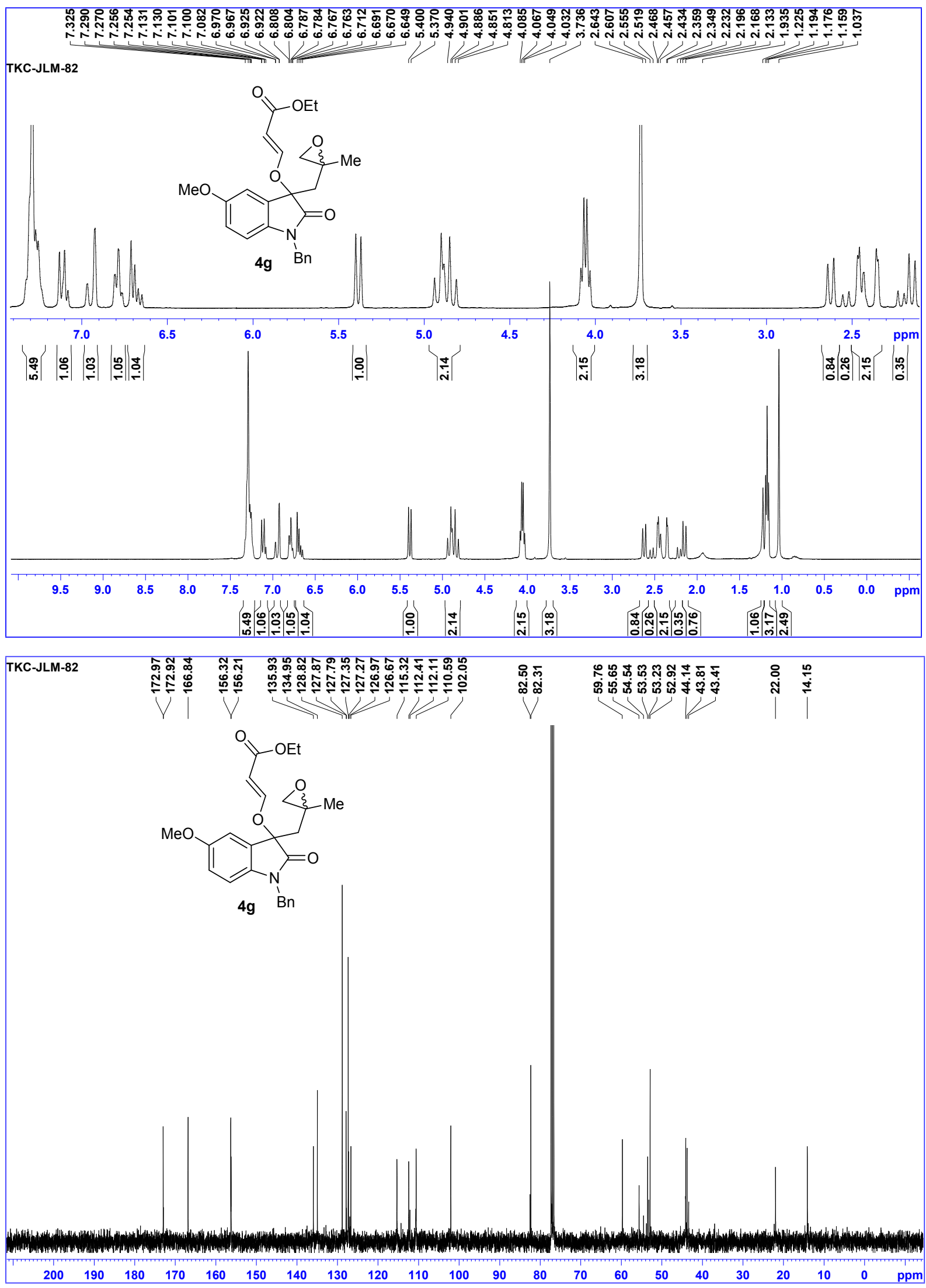
${ }^{1} \mathrm{H}-\mathrm{NMR}\left(\mathrm{CDCl}_{3}, 400 \mathrm{MHz}\right)$ and ${ }^{13} \mathrm{C}-\mathrm{NMR}\left(\mathrm{CDCl}_{3}, 100 \mathrm{MHz}\right)$ spectra of compound $\mathbf{4 h}$
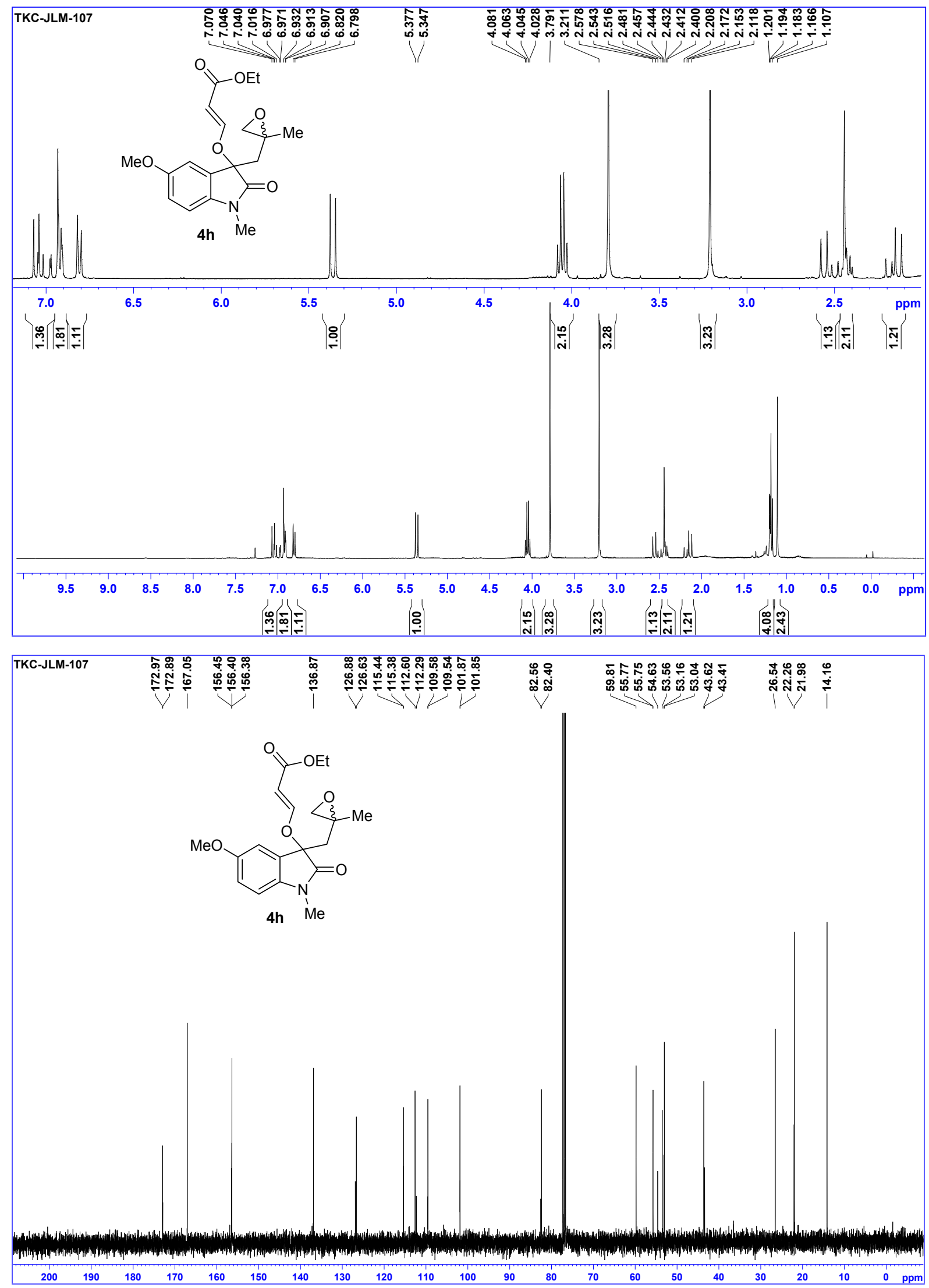
${ }^{1} \mathrm{H}-\mathrm{NMR}\left(\mathrm{CDCl}_{3}, 400 \mathrm{MHz}\right)$ and ${ }^{13} \mathrm{C}-\mathrm{NMR}\left(\mathrm{CDCl}_{3}, 100 \mathrm{MHz}\right)$ spectra of compound $4 \mathbf{i}$
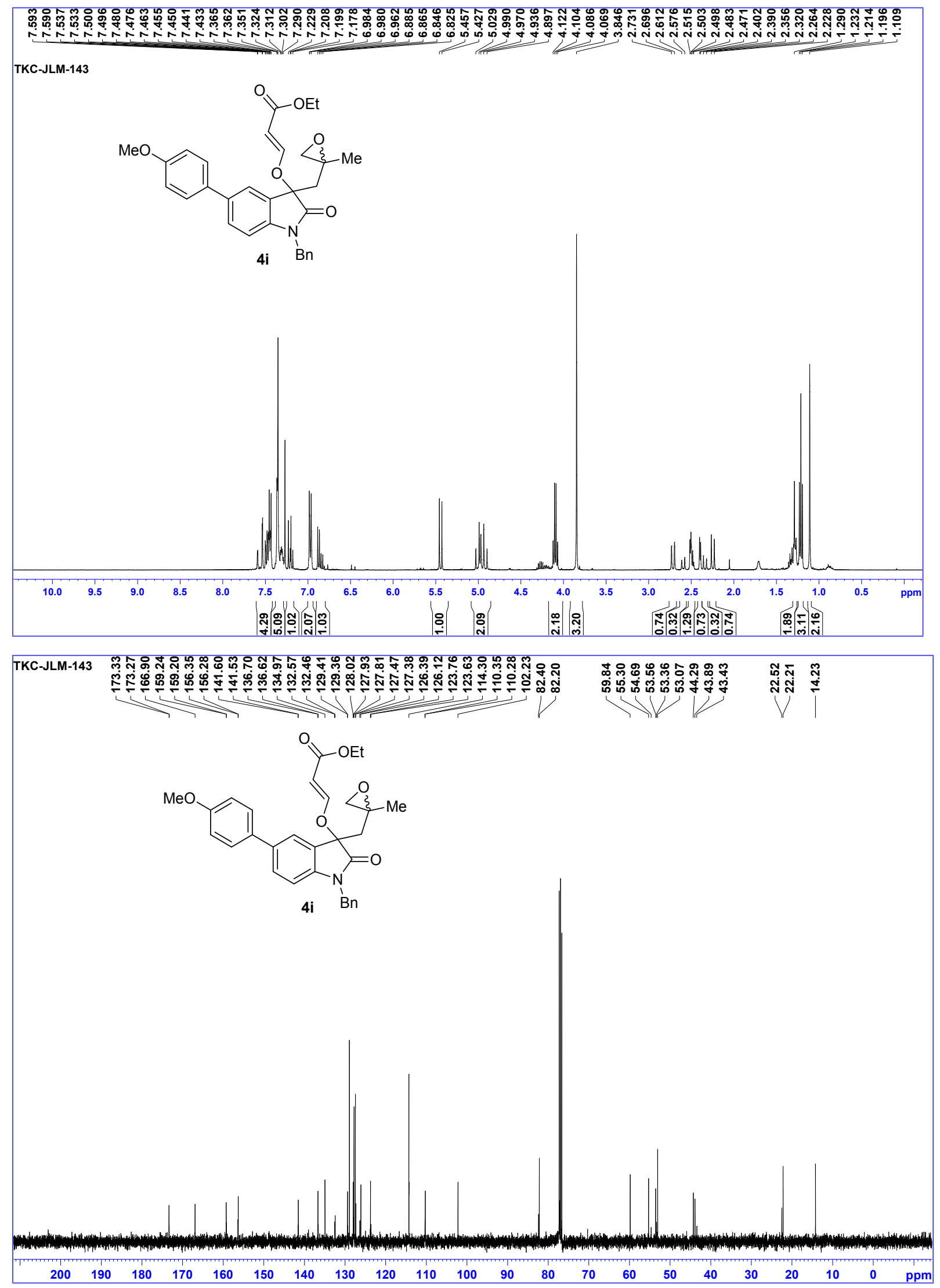
${ }^{1} \mathrm{H}-\mathrm{NMR}\left(\mathrm{CDCl}_{3}, 400 \mathrm{MHz}\right)$ and ${ }^{13} \mathrm{C}-\mathrm{NMR}\left(\mathrm{CDCl}_{3}, 100 \mathrm{MHz}\right)$ spectra of compound $\mathbf{4 j}$
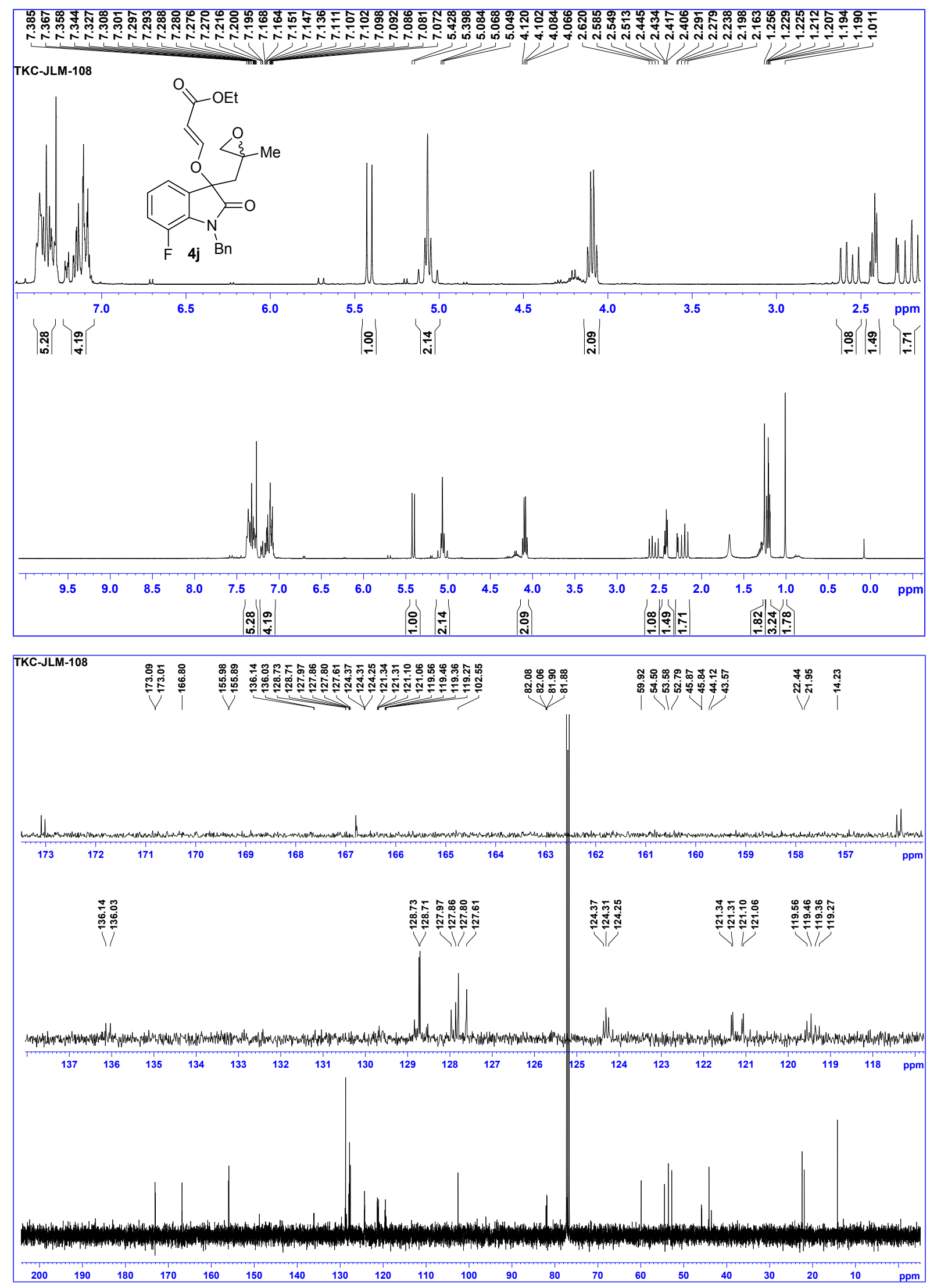
${ }^{1} \mathrm{H}-\mathrm{NMR}\left(\mathrm{CDCl}_{3}, 400 \mathrm{MHz}\right)$ and ${ }^{13} \mathrm{C}-\mathrm{NMR}\left(\mathrm{CDCl}_{3}, 100 \mathrm{MHz}\right)$ spectra of compound 5a
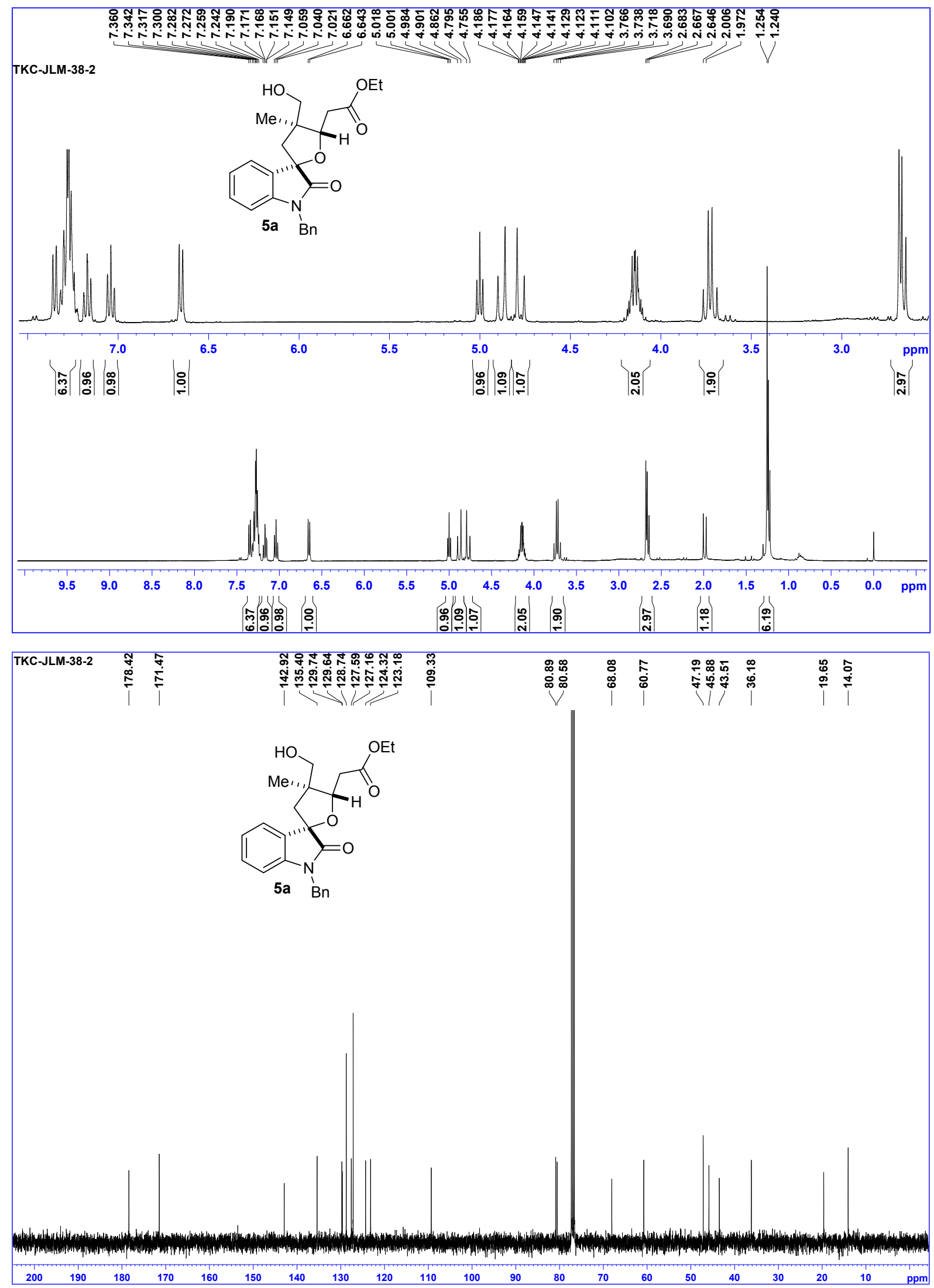
${ }^{1} \mathrm{H}-\mathrm{NMR}\left(\mathrm{CDCl}_{3}, 400 \mathrm{MHz}\right)$ and ${ }^{13} \mathrm{C}-\mathrm{NMR}\left(\mathrm{CDCl}_{3}, 100 \mathrm{MHz}\right)$ spectra of compound 5a'
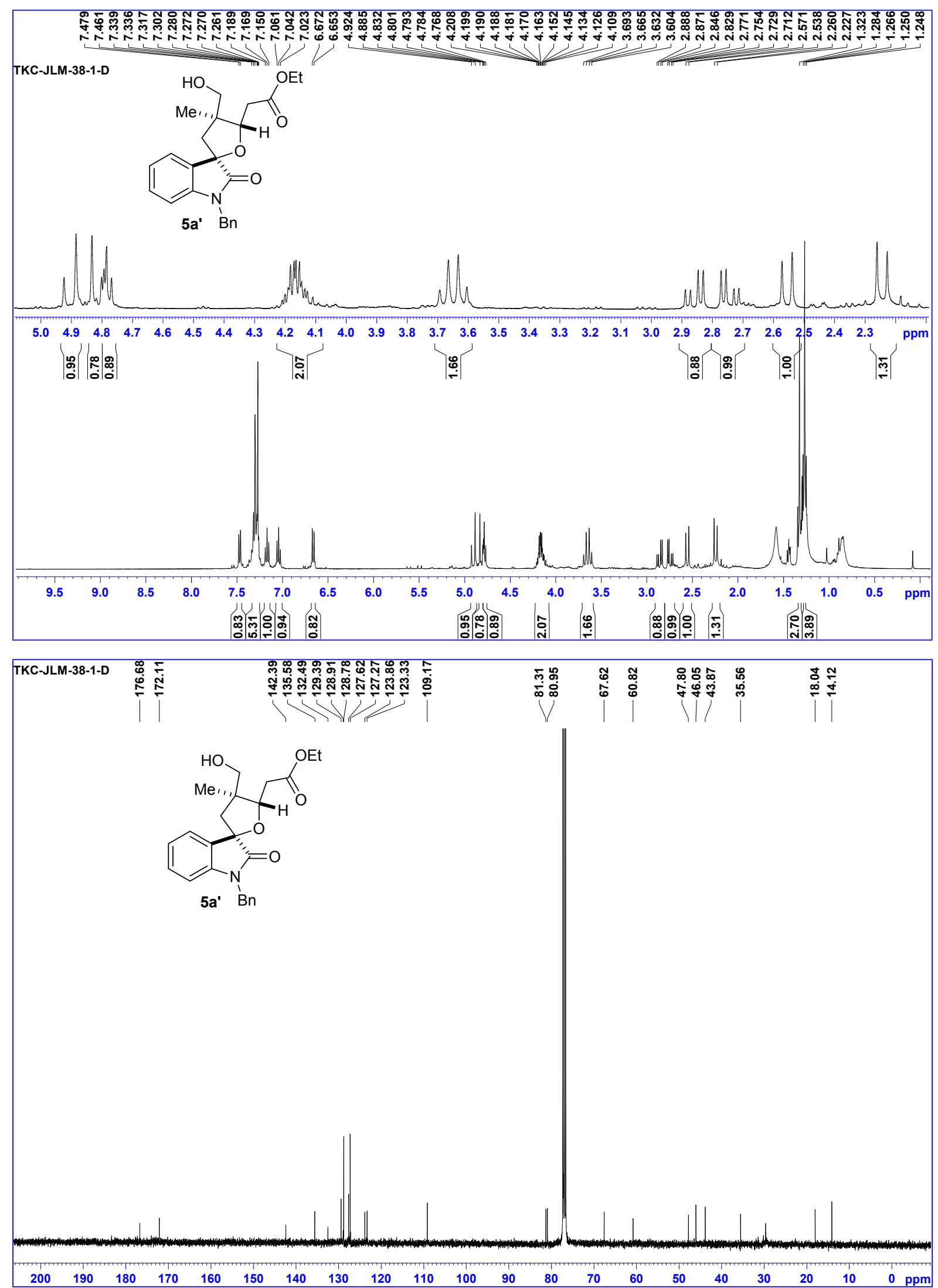
${ }^{1} \mathrm{H}-\mathrm{NMR}\left(\mathrm{CDCl}_{3}, 400 \mathrm{MHz}\right)$ and ${ }^{13} \mathrm{C}-\mathrm{NMR}\left(\mathrm{CDCl}_{3}, 100 \mathrm{MHz}\right)$ spectra of compound $\mathbf{6 a}$
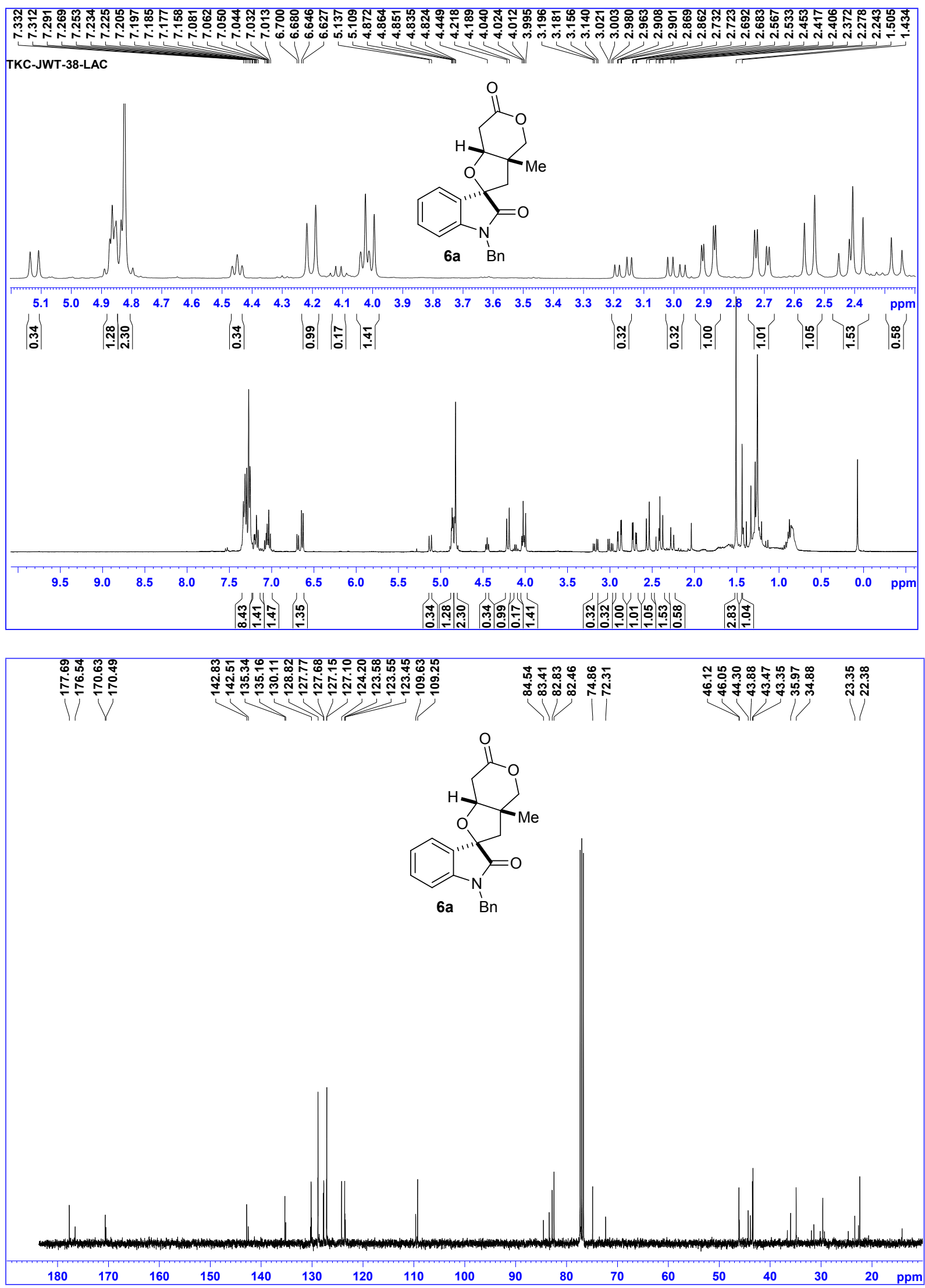
${ }^{1} \mathrm{H}-\mathrm{NMR}\left(\mathrm{CDCl}_{3}, 400 \mathrm{MHz}\right)$ and ${ }^{13} \mathrm{C}-\mathrm{NMR}\left(\mathrm{CDCl}_{3}, 100 \mathrm{MHz}\right)$ spectra of compound $\mathbf{5 b}$
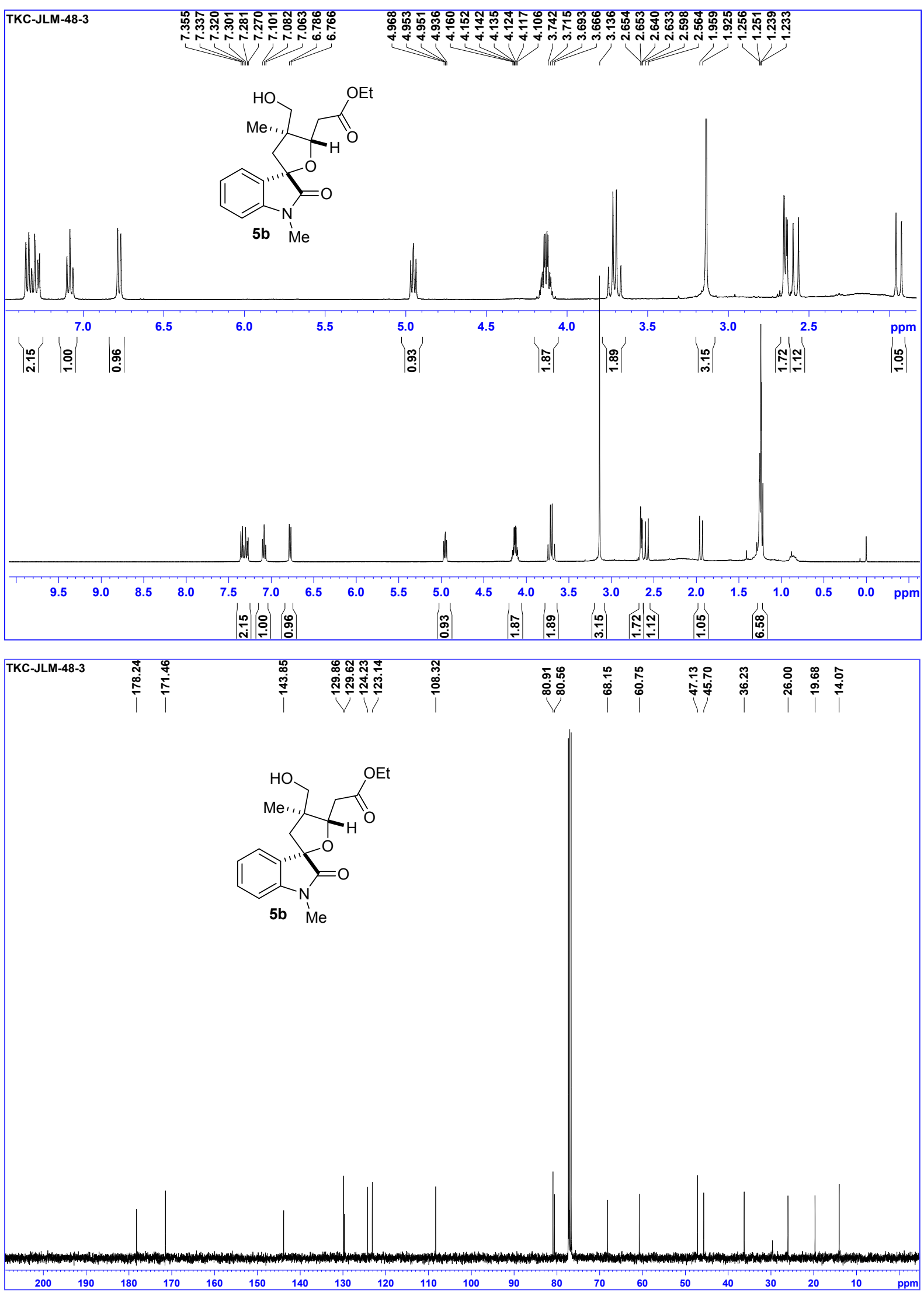
${ }^{1} \mathrm{H}-\mathrm{NMR}\left(\mathrm{CDCl}_{3}, 400 \mathrm{MHz}\right)$ spectra of compound $\mathbf{6 b} / \mathbf{6} \mathbf{b}$
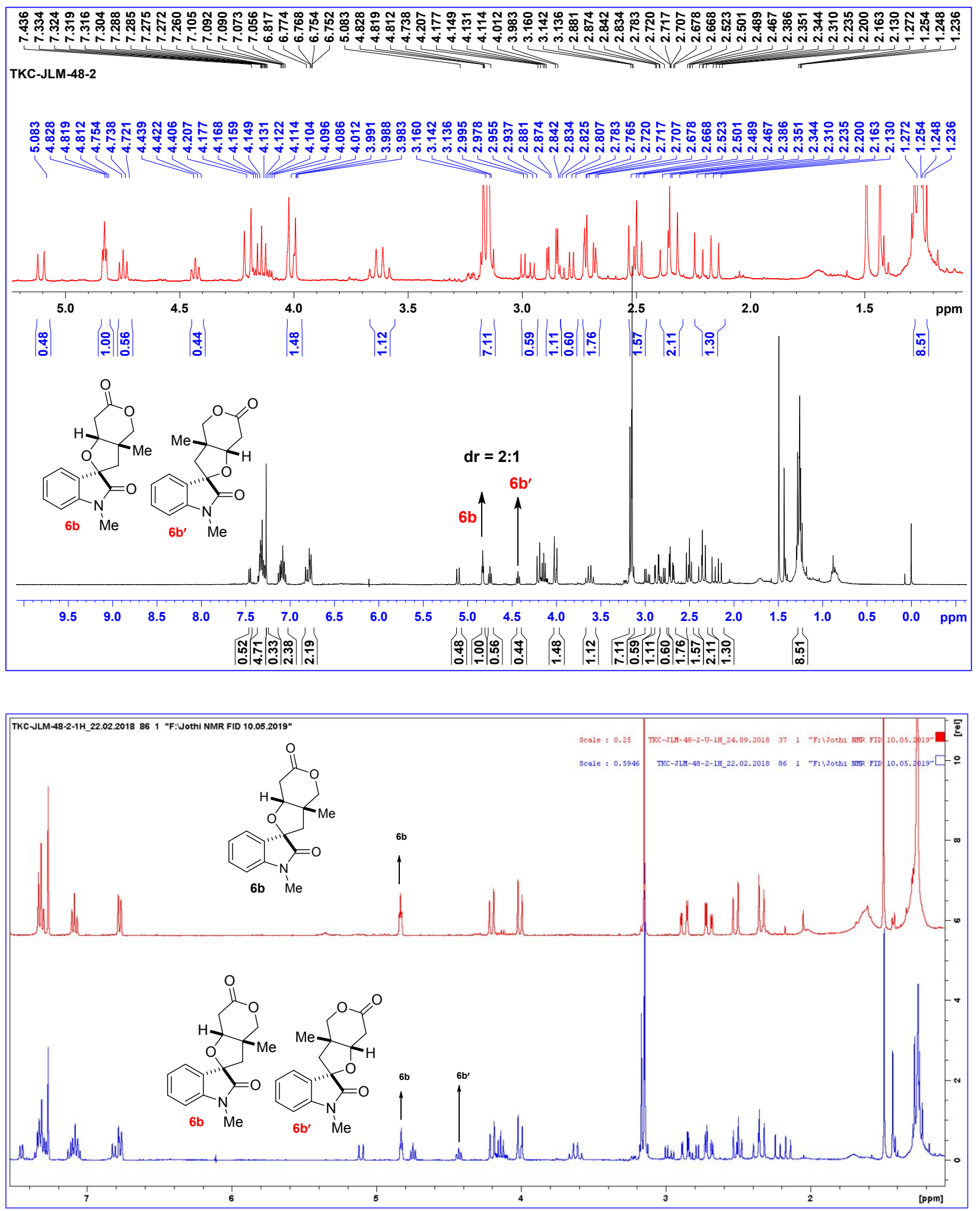
${ }^{1} \mathrm{H}-\mathrm{NMR}\left(\mathrm{CDCl}_{3}, 400 \mathrm{MHz}\right)$ and ${ }^{13} \mathrm{C}-\mathrm{NMR}\left(\mathrm{CDCl}_{3}, 100 \mathrm{MHz}\right)$ spectra of compound $\mathbf{6 b}$
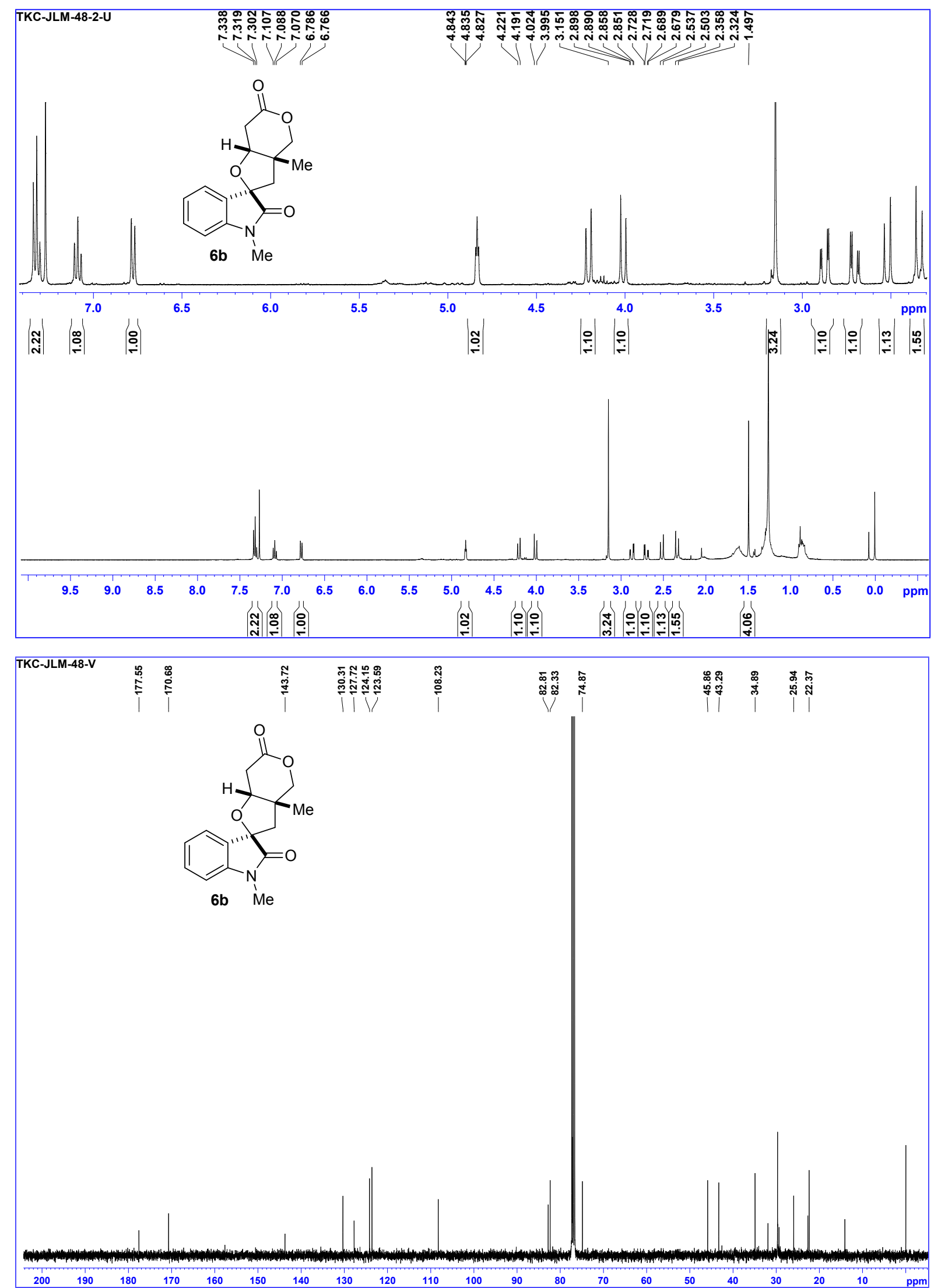
$\operatorname{NOE}\left(\mathrm{CDCl}_{3}, 400 \mathrm{MHz}\right)$ spectra of compound $\mathbf{5 b}$ and $\mathbf{6 b}$
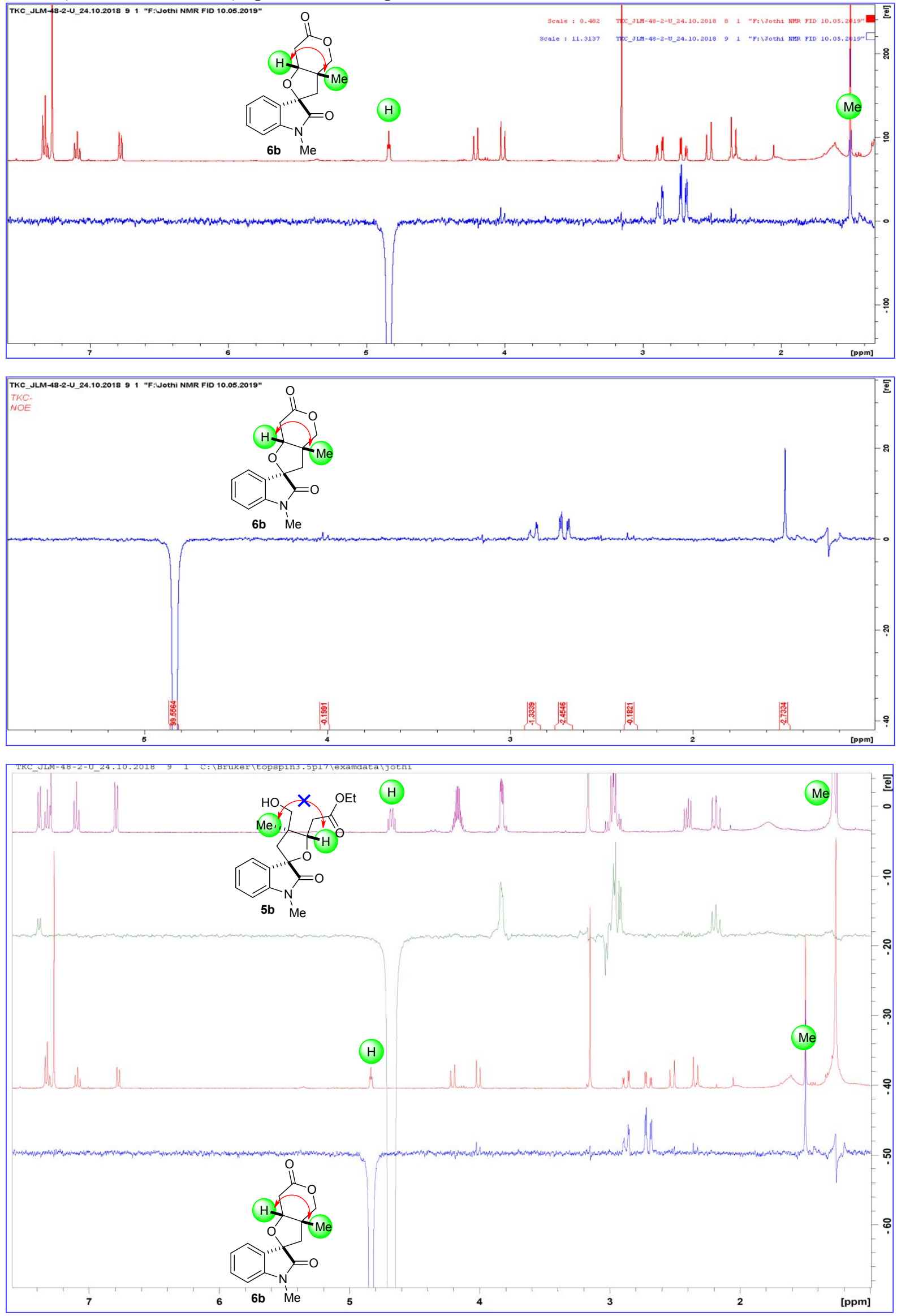
${ }^{1} \mathrm{H}-\mathrm{NMR}\left(\mathrm{CDCl}_{3}, 400 \mathrm{MHz}\right)$ and ${ }^{13} \mathrm{C}-\mathrm{NMR}\left(\mathrm{CDCl}_{3}, 100 \mathrm{MHz}\right)$ spectra of compound $\mathbf{5 c}$
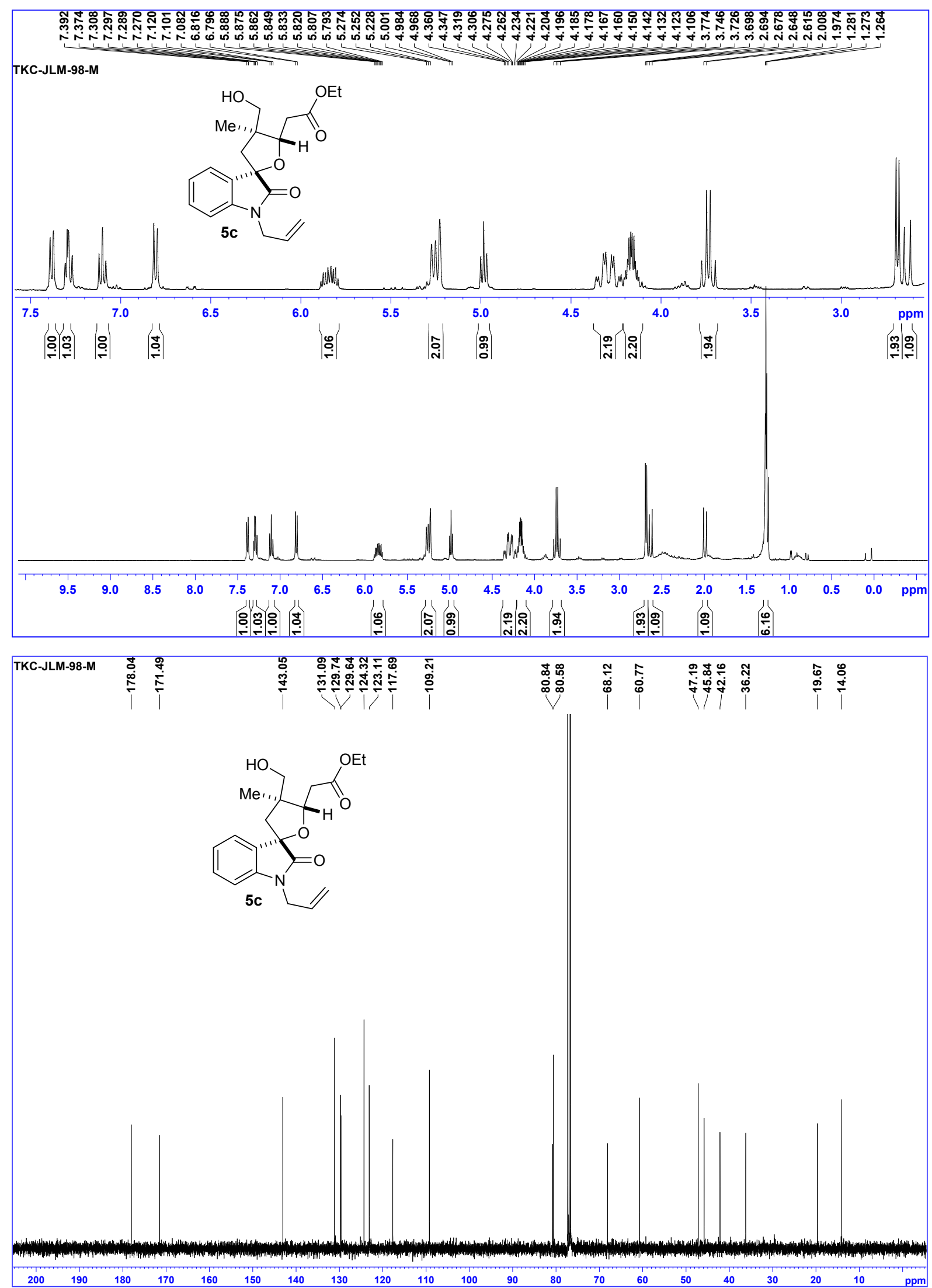
${ }^{1} \mathrm{H}-\mathrm{NMR}\left(\mathrm{CDCl}_{3}, 400 \mathrm{MHz}\right)$ and ${ }^{13} \mathrm{C}-\mathrm{NMR}\left(\mathrm{CDCl}_{3}, 100 \mathrm{MHz}\right)$ spectra of compound $\mathbf{6 c}$
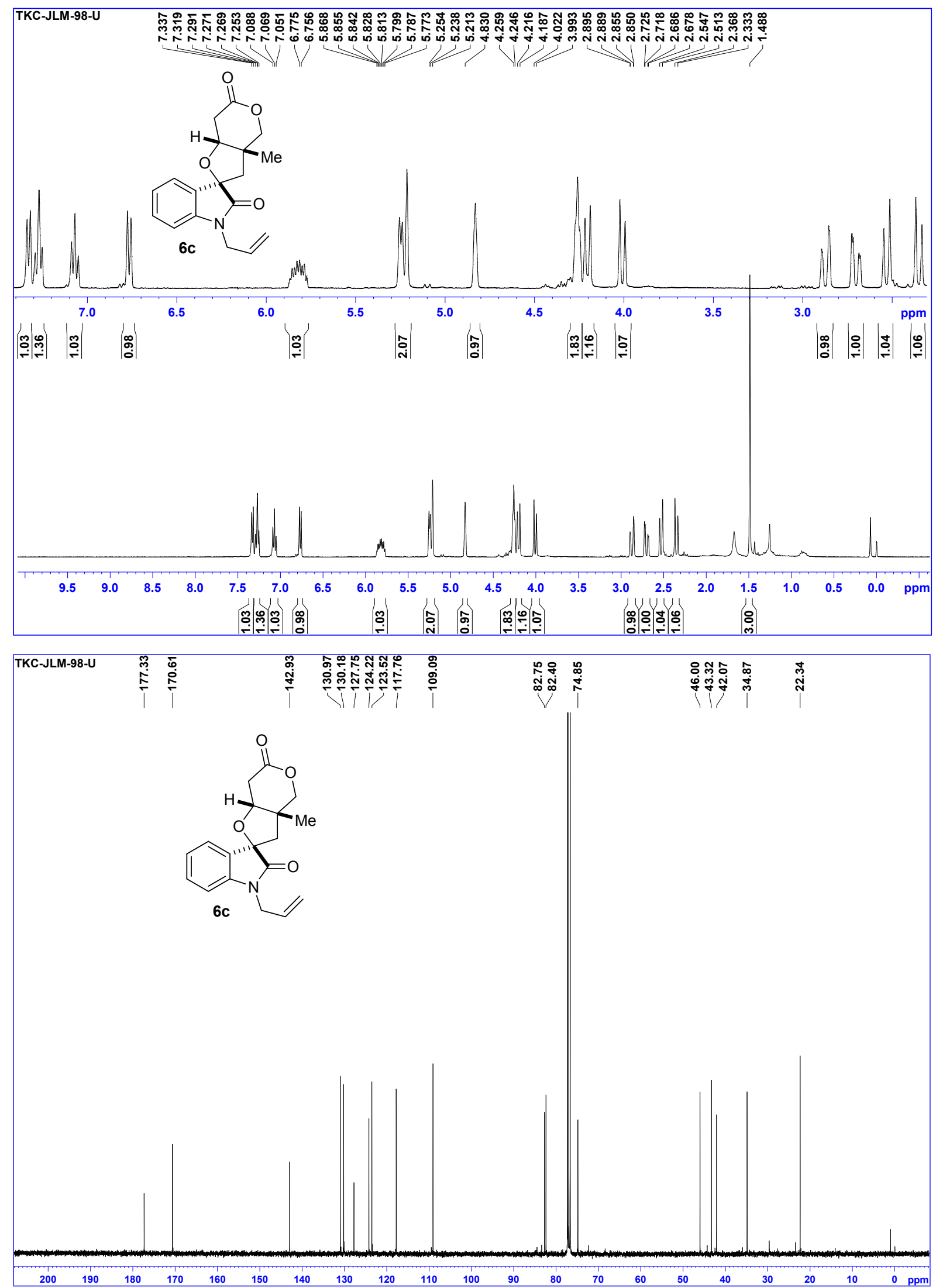
NOESY $\left(\mathrm{CDCl}_{3}, 400 \mathrm{MHz}\right)$ spectra of compound $\mathbf{6 c}$

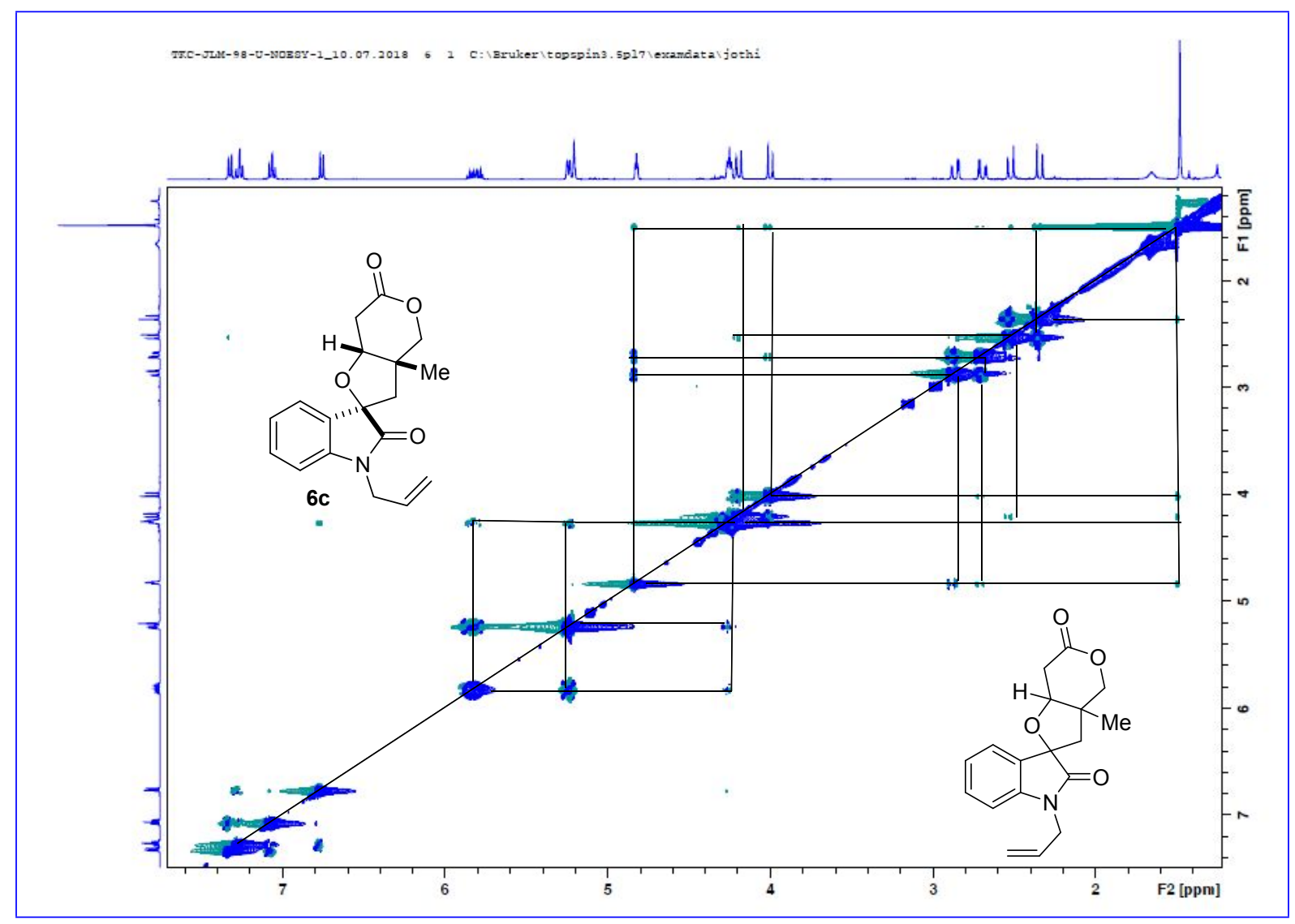


${ }^{1} \mathrm{H}-\mathrm{NMR}\left(\mathrm{CDCl}_{3}, 400 \mathrm{MHz}\right)$ and ${ }^{13} \mathrm{C}-\mathrm{NMR}\left(\mathrm{CDCl}_{3}, 100 \mathrm{MHz}\right)$ spectra of compound $\mathbf{5 d}$
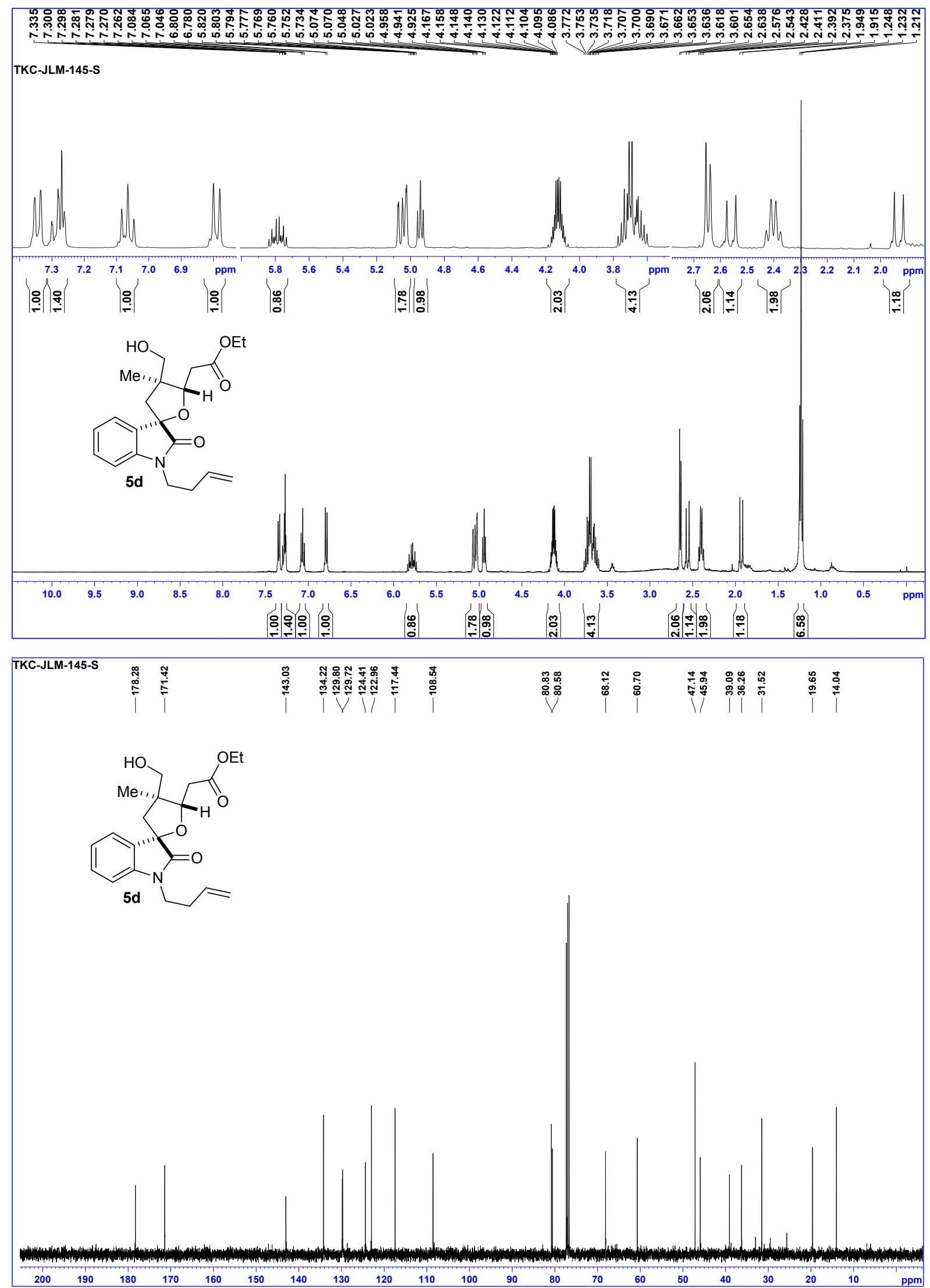
${ }^{1} \mathrm{H}-\mathrm{NMR}\left(\mathrm{CDCl}_{3}, 400 \mathrm{MHz}\right)$ and ${ }^{13} \mathrm{C}-\mathrm{NMR}\left(\mathrm{CDCl}_{3}, 100 \mathrm{MHz}\right)$ spectra of compound $\mathbf{6 d}$
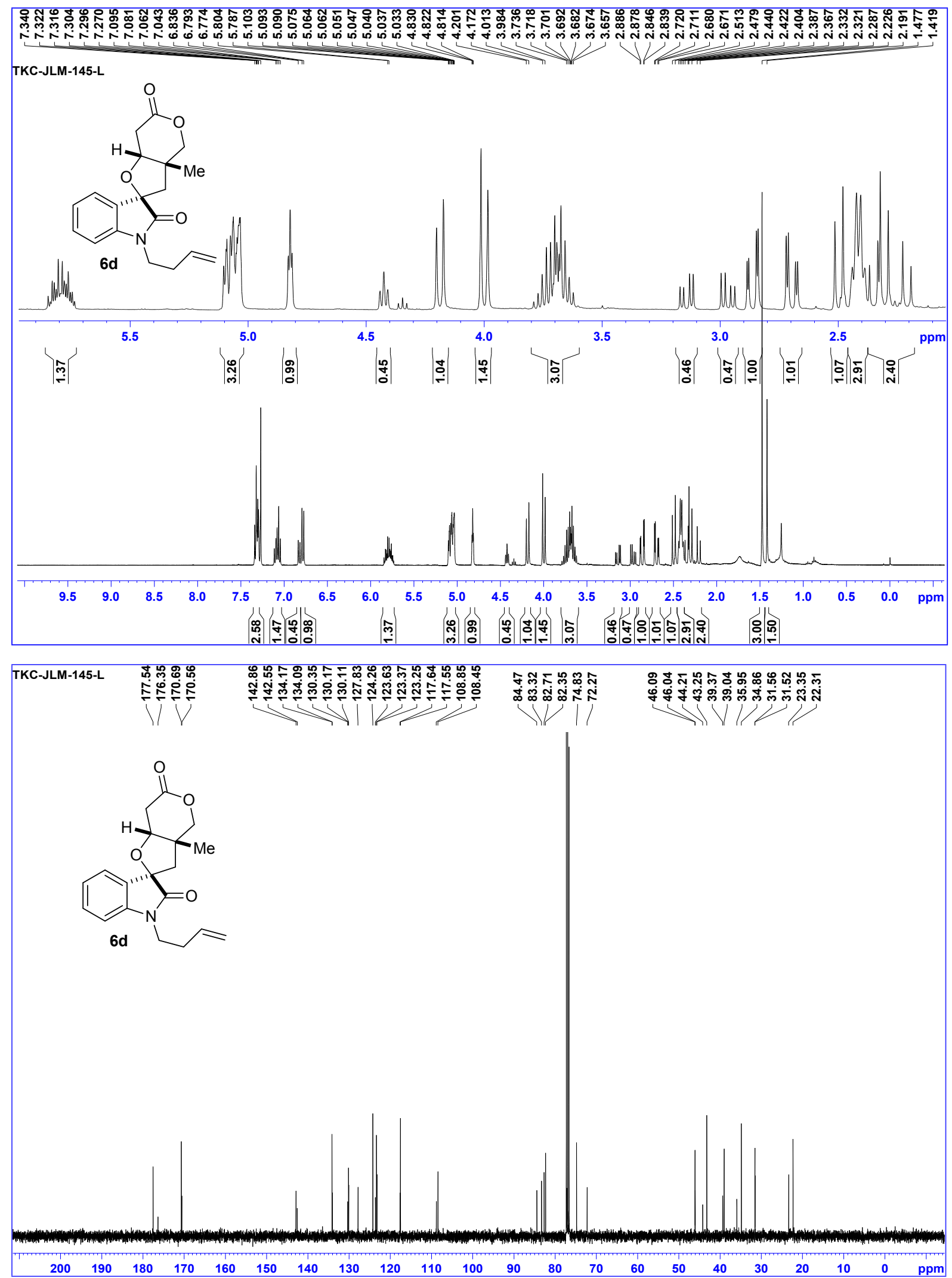
${ }^{1} \mathrm{H}-\mathrm{NMR}\left(\mathrm{CDCl}_{3}, 400 \mathrm{MHz}\right)$ and ${ }^{13} \mathrm{C}-\mathrm{NMR}\left(\mathrm{CDCl}_{3}, 100 \mathrm{MHz}\right)$ spectra of compound $\mathbf{5 e}$
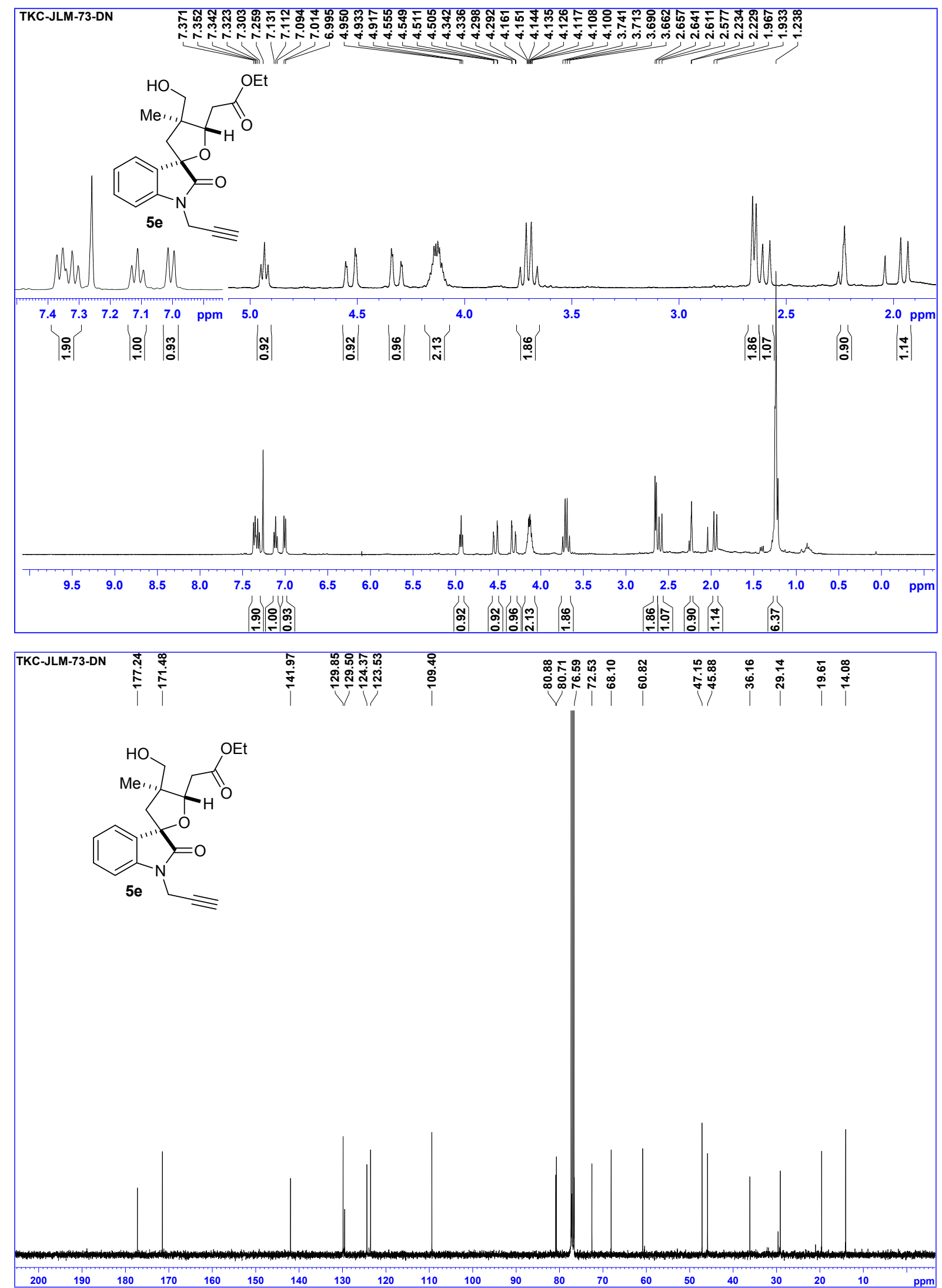
${ }^{1} \mathrm{H}-\mathrm{NMR}\left(\mathrm{CDCl}_{3}, 400 \mathrm{MHz}\right)$ and ${ }^{13} \mathrm{C}-\mathrm{NMR}\left(\mathrm{CDCl}_{3}, 100 \mathrm{MHz}\right)$ spectra of compound $6 \mathbf{e}$
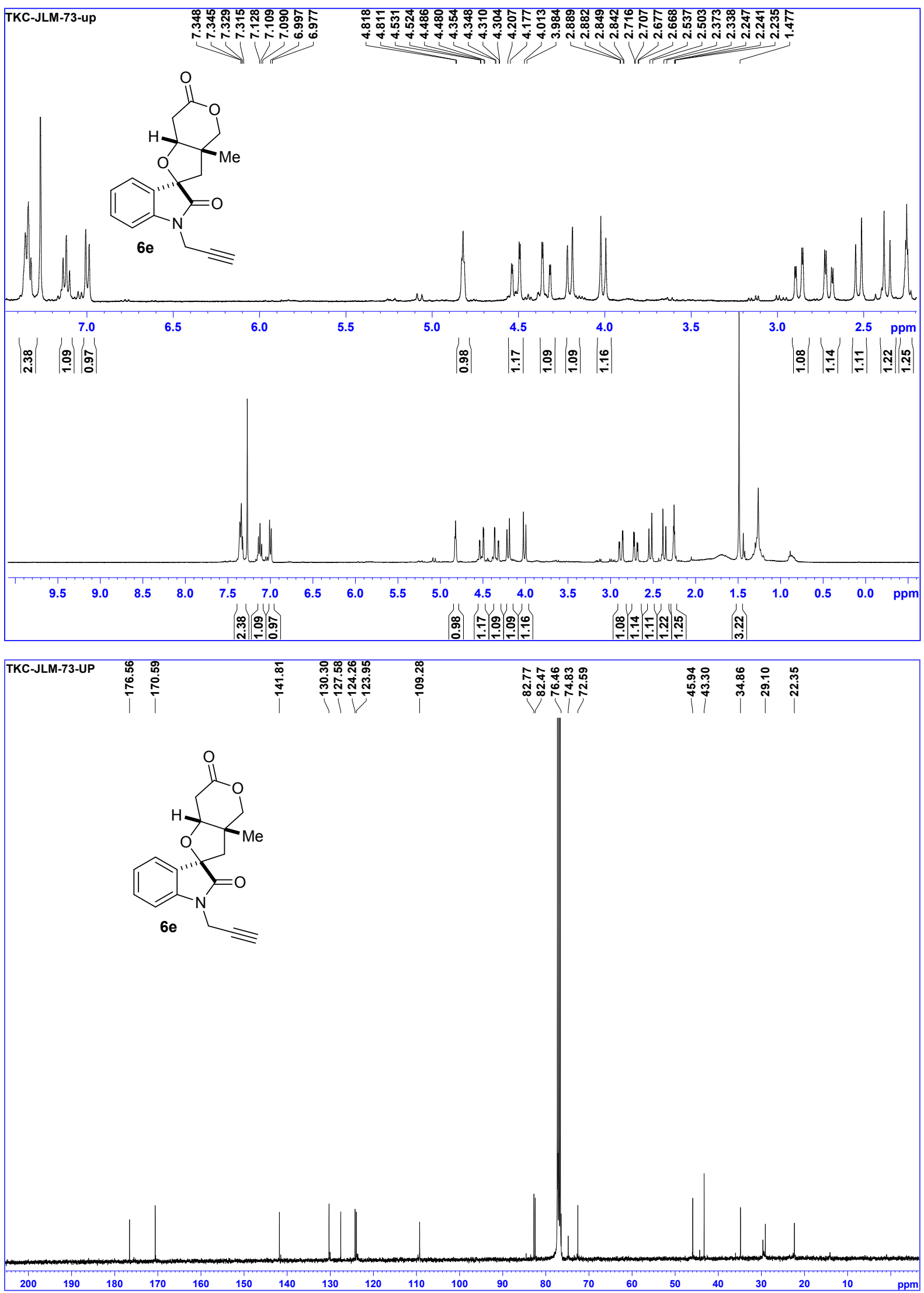
$\mathrm{HSQC}$ and $\mathrm{HMBC}\left(\mathrm{CDCl}_{3}, 400 \mathrm{MHz}\right)$ spectra of compound $\mathbf{6 e}$
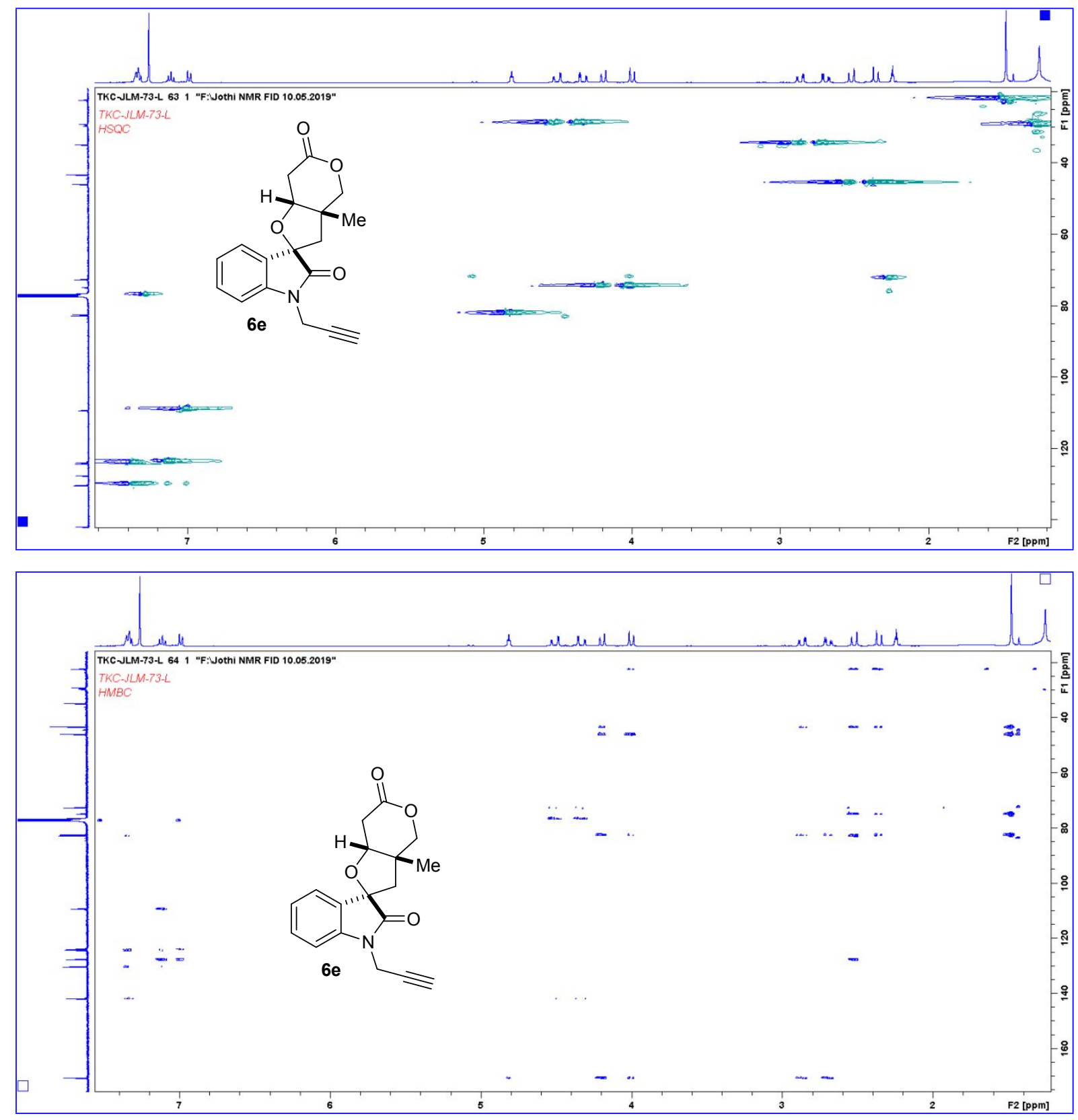
${ }^{1} \mathrm{H}-\mathrm{NMR}\left(\mathrm{CDCl}_{3}, 400 \mathrm{MHz}\right)$ and ${ }^{13} \mathrm{C}-\mathrm{NMR}\left(\mathrm{CDCl}_{3}, 100 \mathrm{MHz}\right)$ spectra of compound $\mathbf{5 f}$
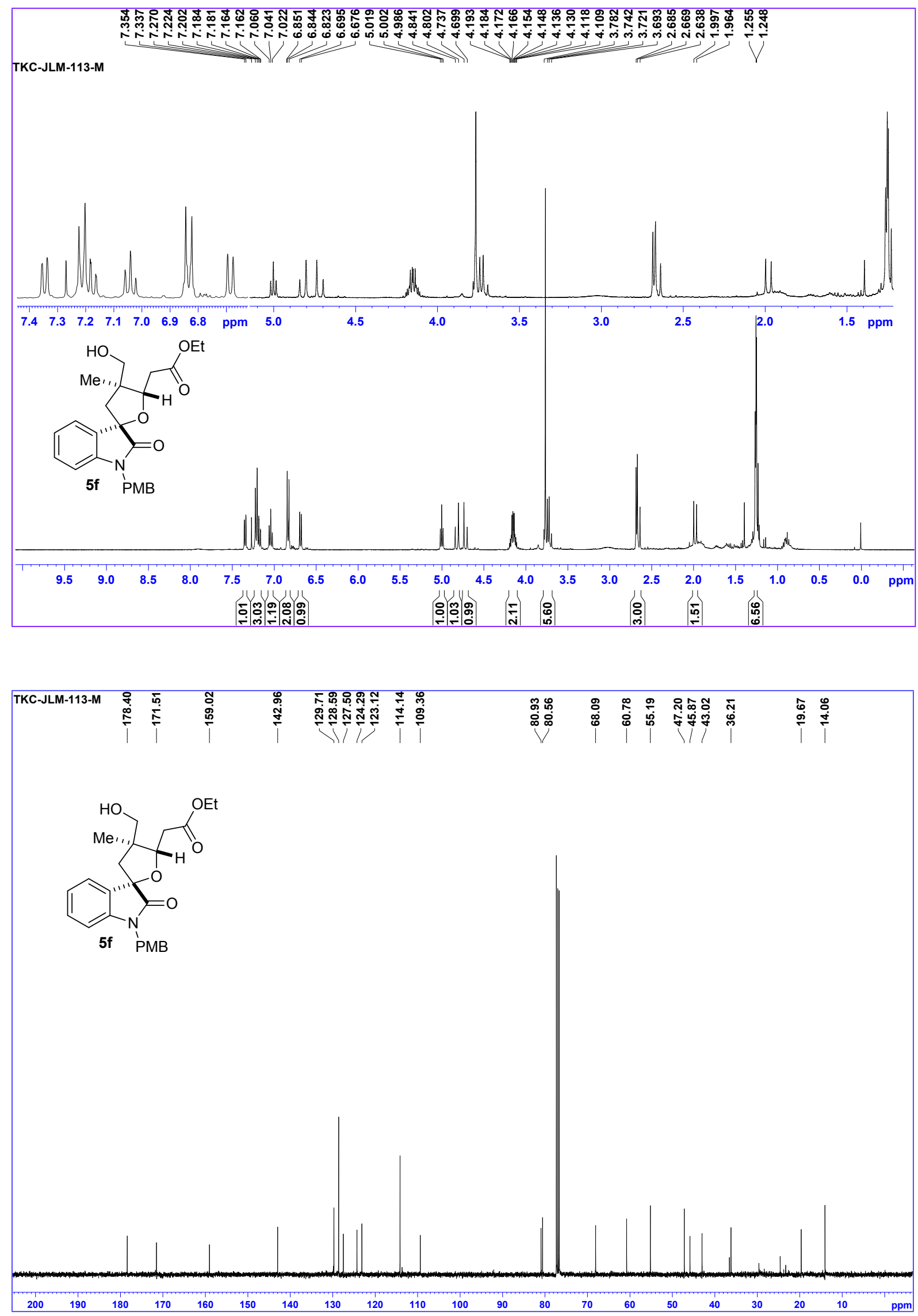
${ }^{1} \mathrm{H}-\mathrm{NMR}\left(\mathrm{CDCl}_{3}, 400 \mathrm{MHz}\right)$ and ${ }^{13} \mathrm{C}-\mathrm{NMR}\left(\mathrm{CDCl}_{3}, 100 \mathrm{MHz}\right)$ spectra of compound $\mathbf{6} \mathbf{f}$
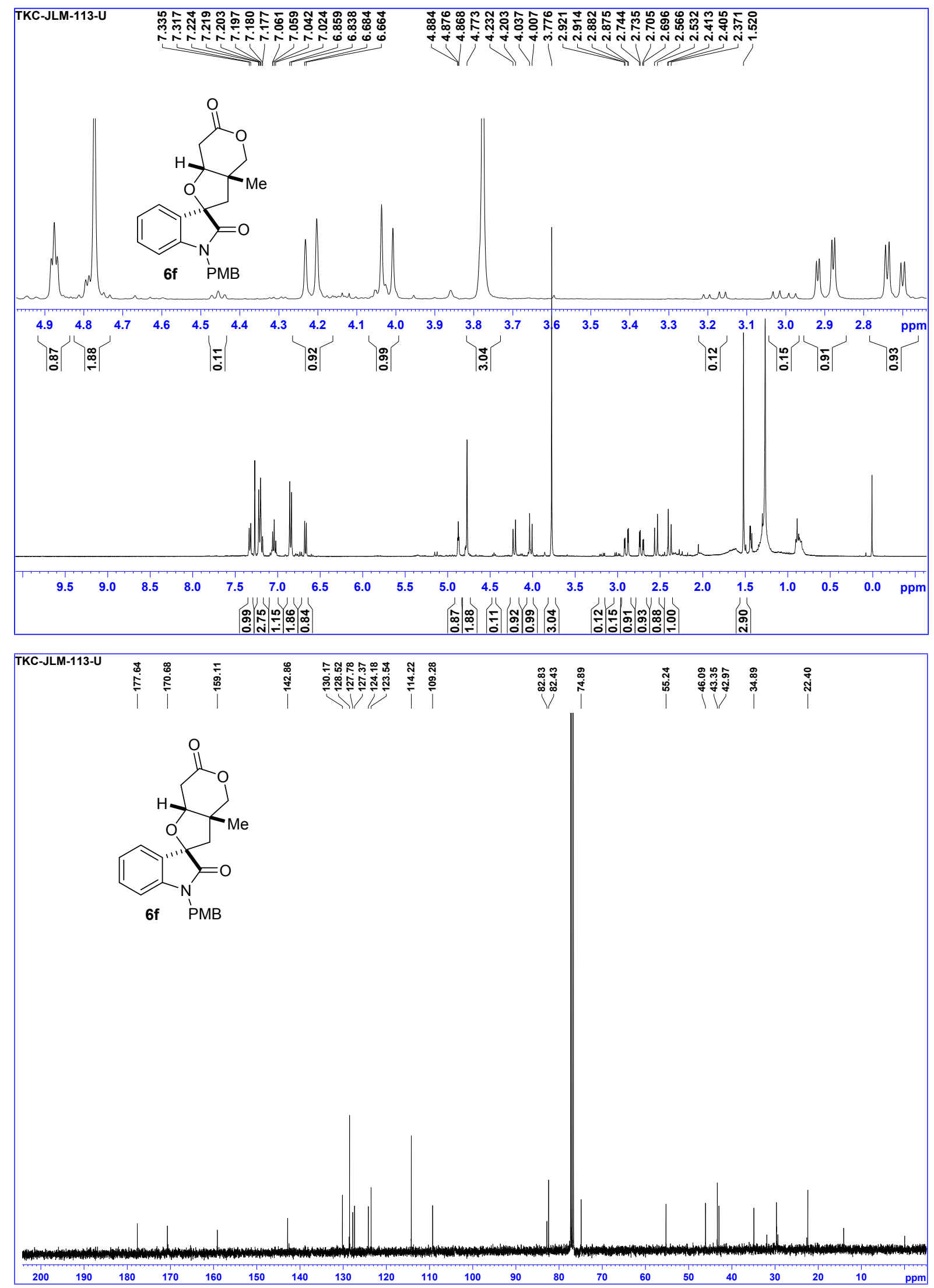
${ }^{1} \mathrm{H}-\mathrm{NMR}\left(\mathrm{CDCl}_{3}, 400 \mathrm{MHz}\right)$ and ${ }^{13} \mathrm{C}-\mathrm{NMR}\left(\mathrm{CDCl}_{3}, 100 \mathrm{MHz}\right)$ spectra of compound $\mathbf{5 g}$
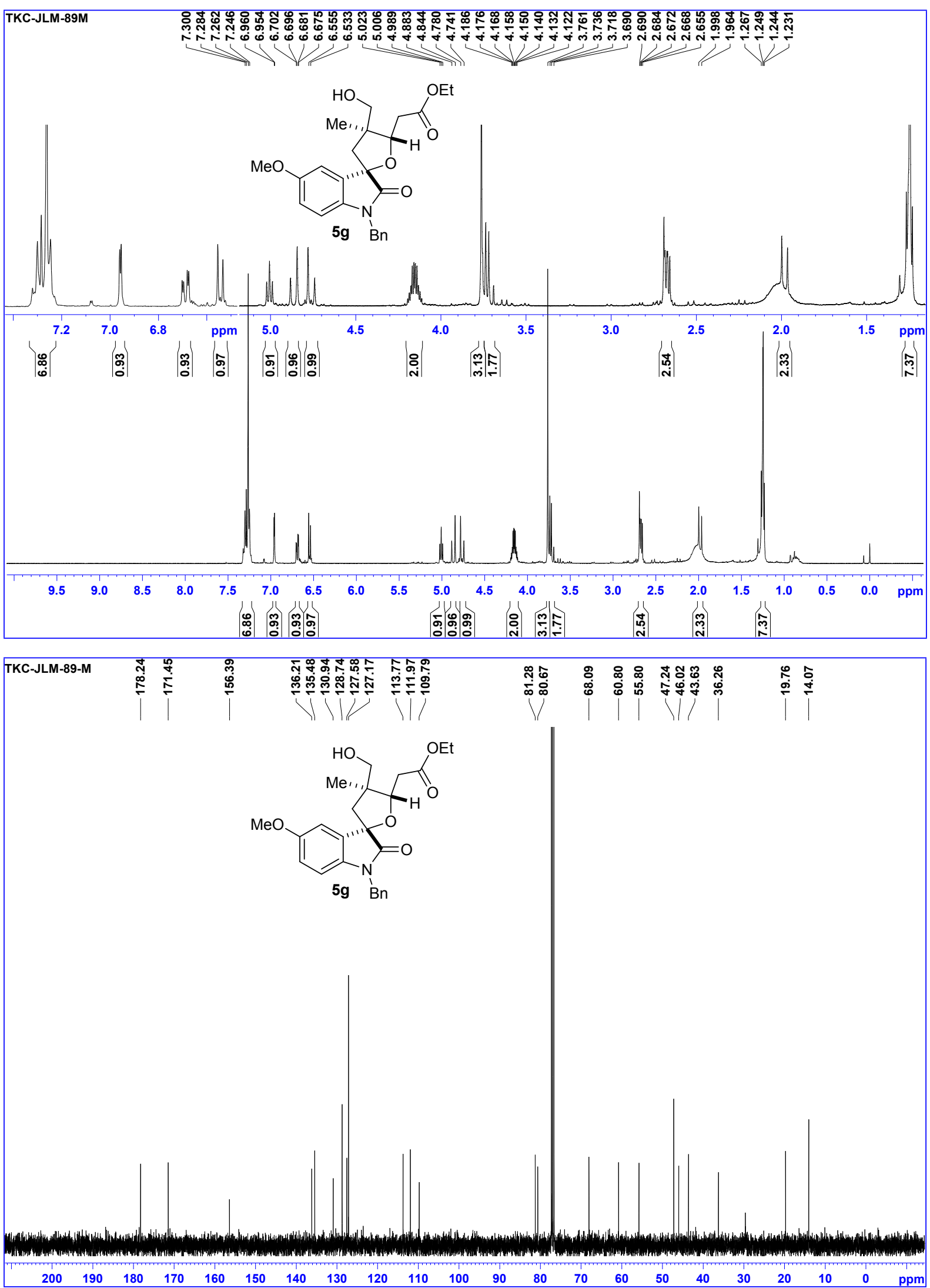
${ }^{1} \mathrm{H}-\mathrm{NMR}\left(\mathrm{CDCl}_{3}, 400 \mathrm{MHz}\right)$ and ${ }^{13} \mathrm{C}-\mathrm{NMR}\left(\mathrm{CDCl}_{3}, 100 \mathrm{MHz}\right)$ spectra of compound $\mathbf{5 g} \mathbf{g}^{\prime \prime}$
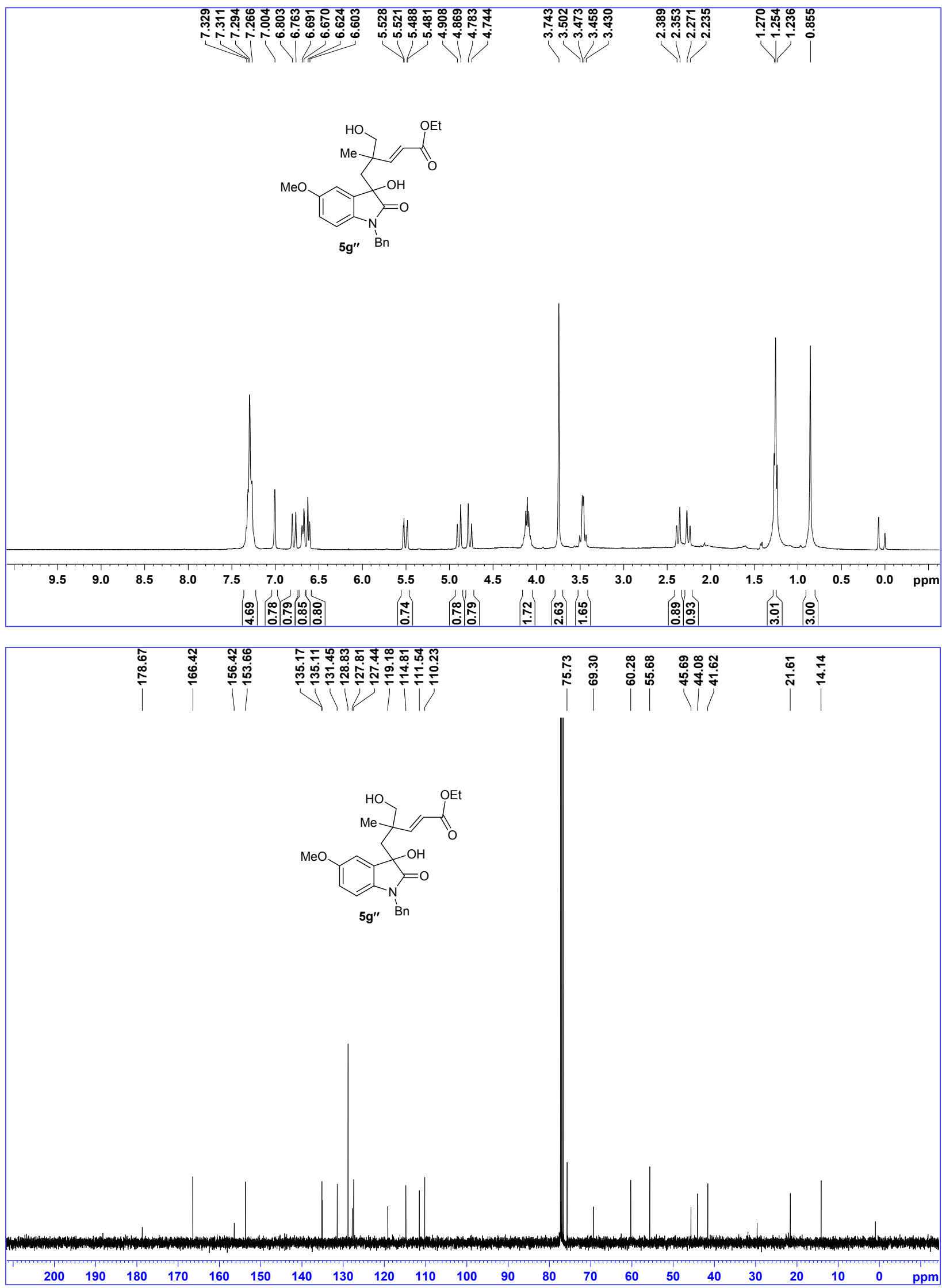
${ }^{1} \mathrm{H}-\mathrm{NMR}\left(\mathrm{CDCl}_{3}, 400 \mathrm{MHz}\right)$ and ${ }^{13} \mathrm{C}-\mathrm{NMR}\left(\mathrm{CDCl}_{3}, 100 \mathrm{MHz}\right)$ spectra of compound $\mathbf{6 g}$
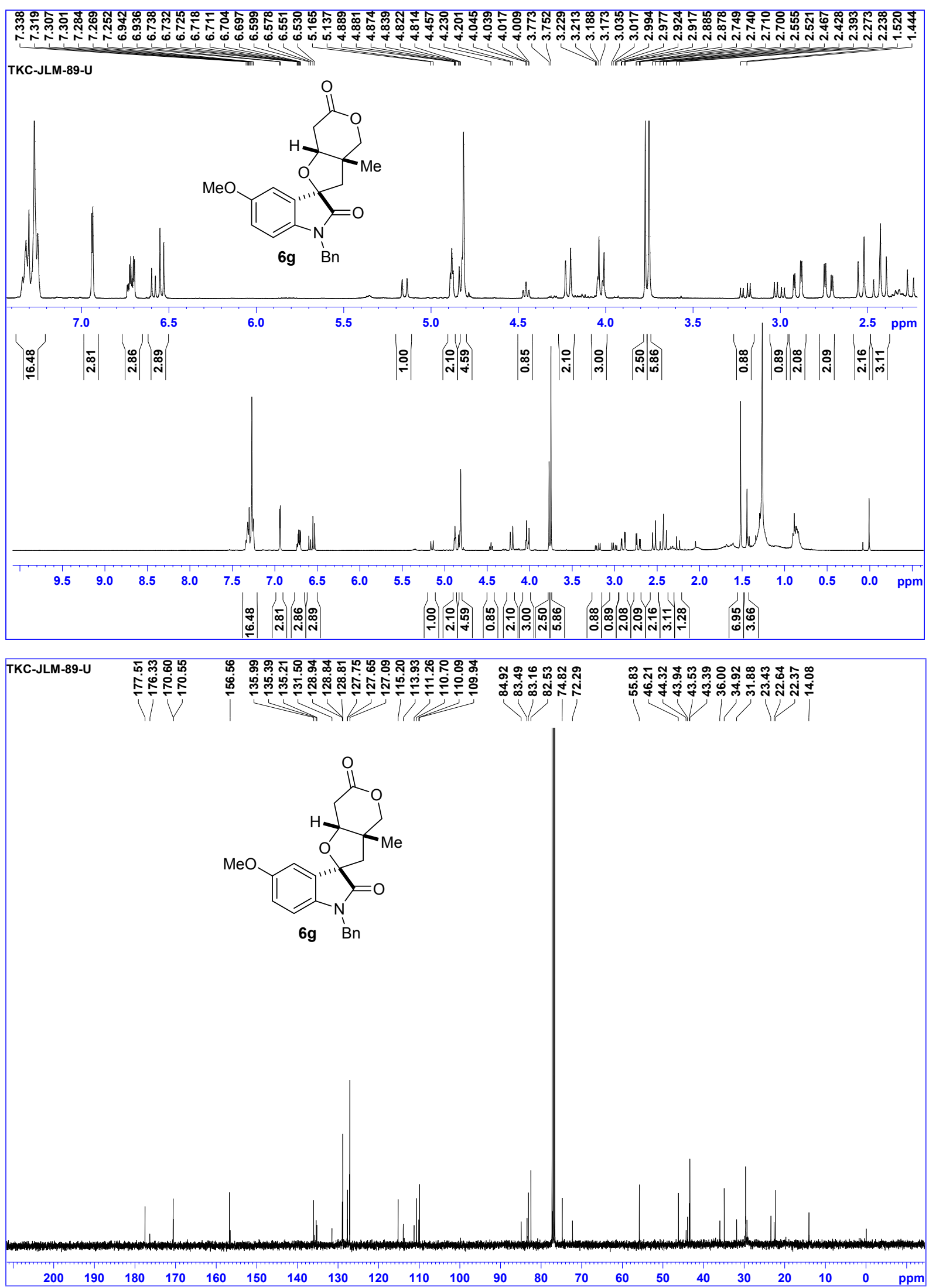
${ }^{1} \mathrm{H}-\mathrm{NMR}\left(\mathrm{CDCl}_{3}, 400 \mathrm{MHz}\right)$ and ${ }^{13} \mathrm{C}-\mathrm{NMR}\left(\mathrm{CDCl}_{3}, 100 \mathrm{MHz}\right)$ spectra of compound $\mathbf{5 h}$

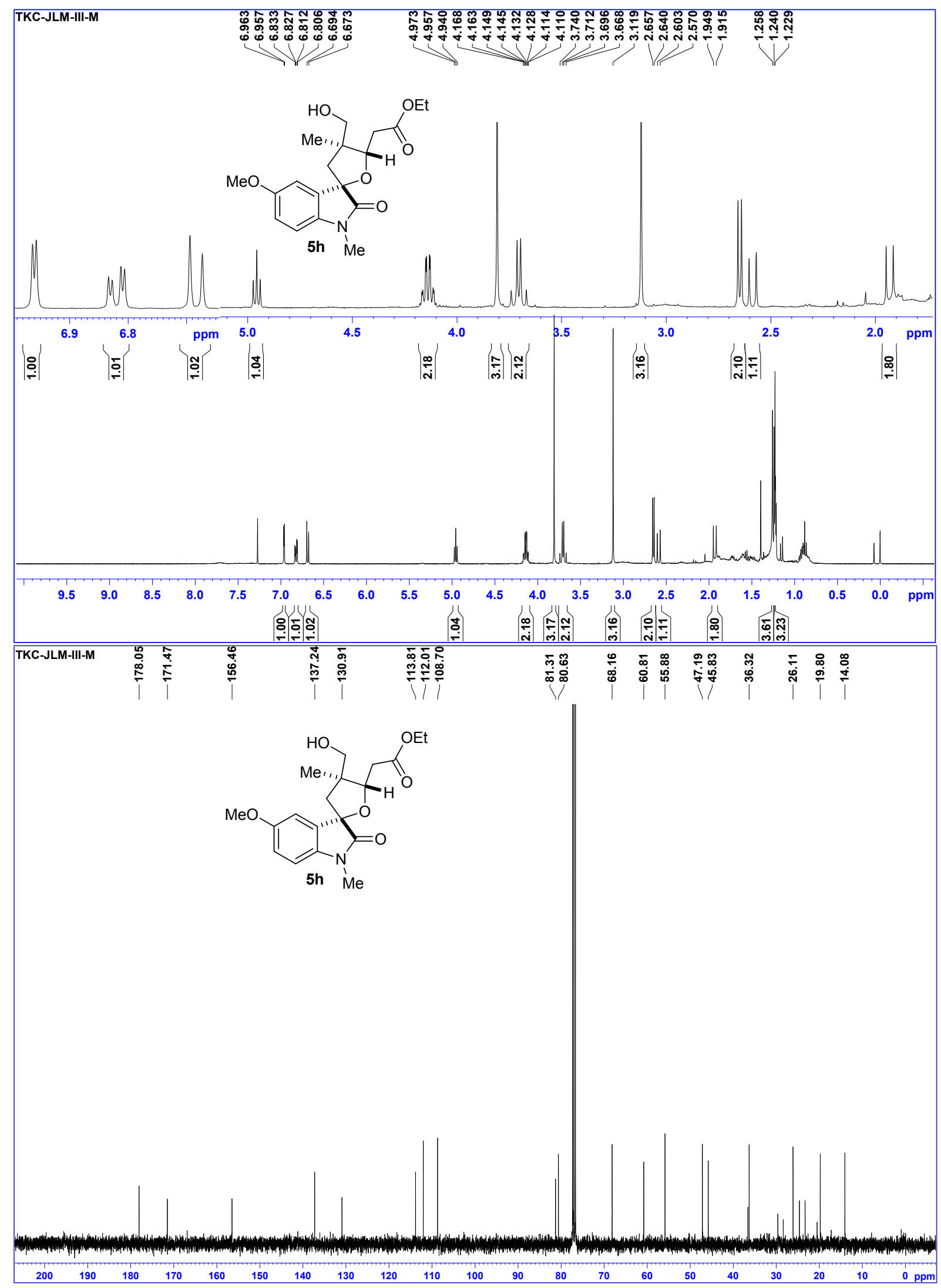


${ }^{1} \mathrm{H}-\mathrm{NMR}\left(\mathrm{CDCl}_{3}, 400 \mathrm{MHz}\right)$ and ${ }^{13} \mathrm{C}-\mathrm{NMR}\left(\mathrm{CDCl}_{3}, 100 \mathrm{MHz}\right)$ spectra of compound $\mathbf{6 h}$
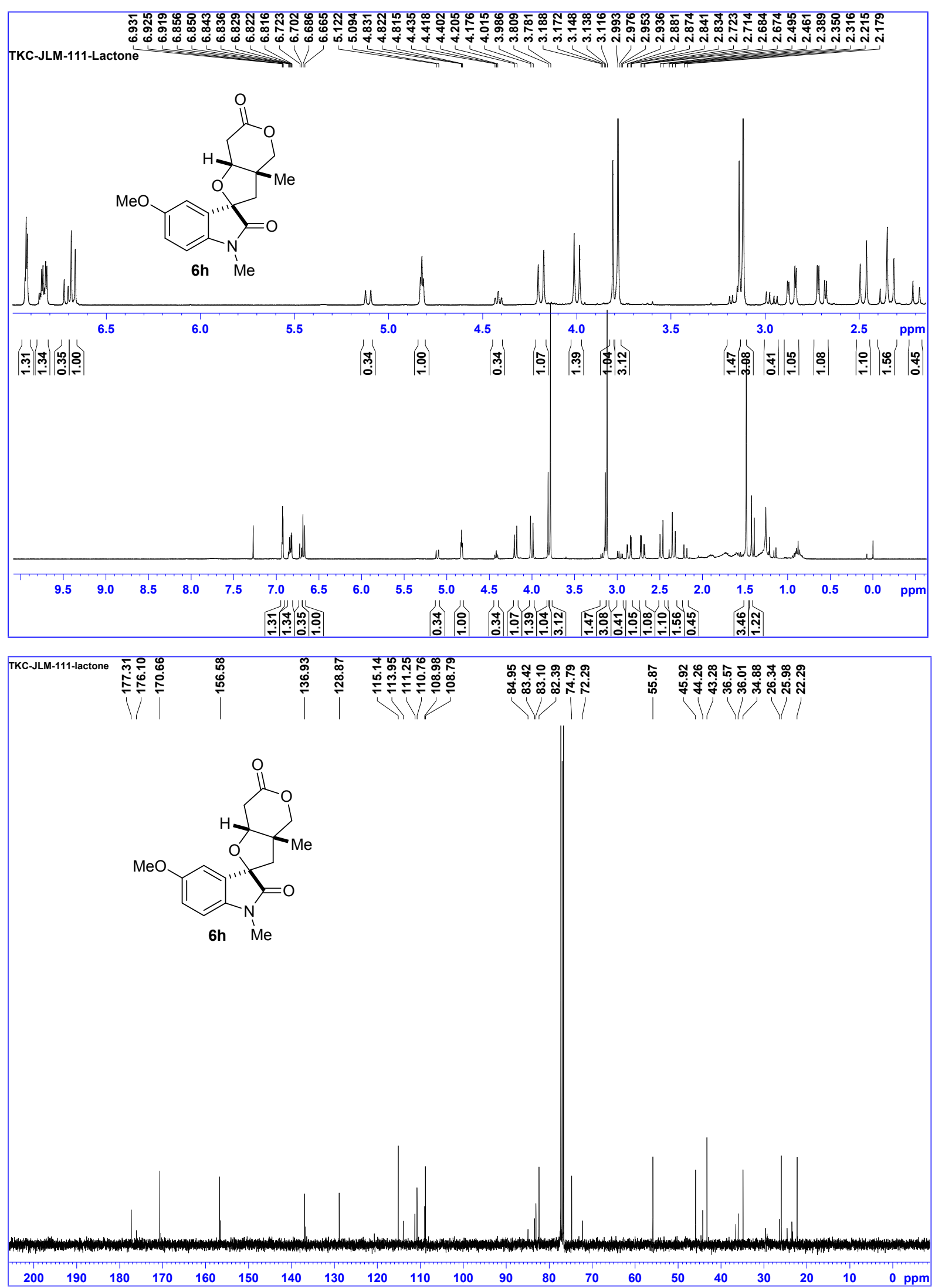
${ }^{1} \mathrm{H}-\mathrm{NMR}\left(\mathrm{CDCl}_{3}, 400 \mathrm{MHz}\right)$ and ${ }^{13} \mathrm{C}-\mathrm{NMR}\left(\mathrm{CDCl}_{3}, 100 \mathrm{MHz}\right)$ spectra of compound $\mathbf{5 i}$
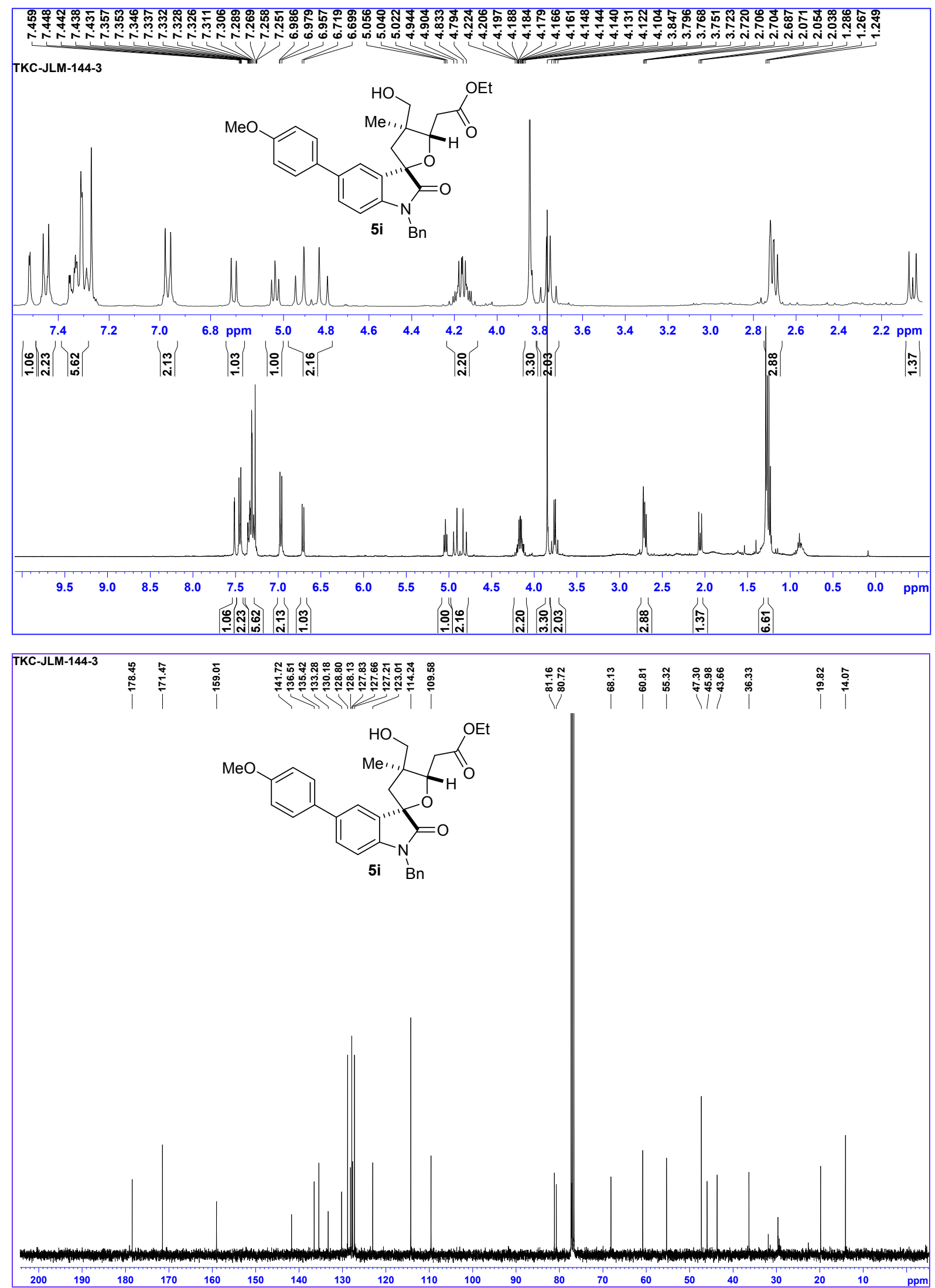
${ }^{1} \mathrm{H}-\mathrm{NMR}\left(\mathrm{CDCl}_{3}, 400 \mathrm{MHz}\right)$ and ${ }^{13} \mathrm{C}-\mathrm{NMR}\left(\mathrm{CDCl}_{3}, 100 \mathrm{MHz}\right)$ spectra of compound $\mathbf{6} \mathbf{i}$
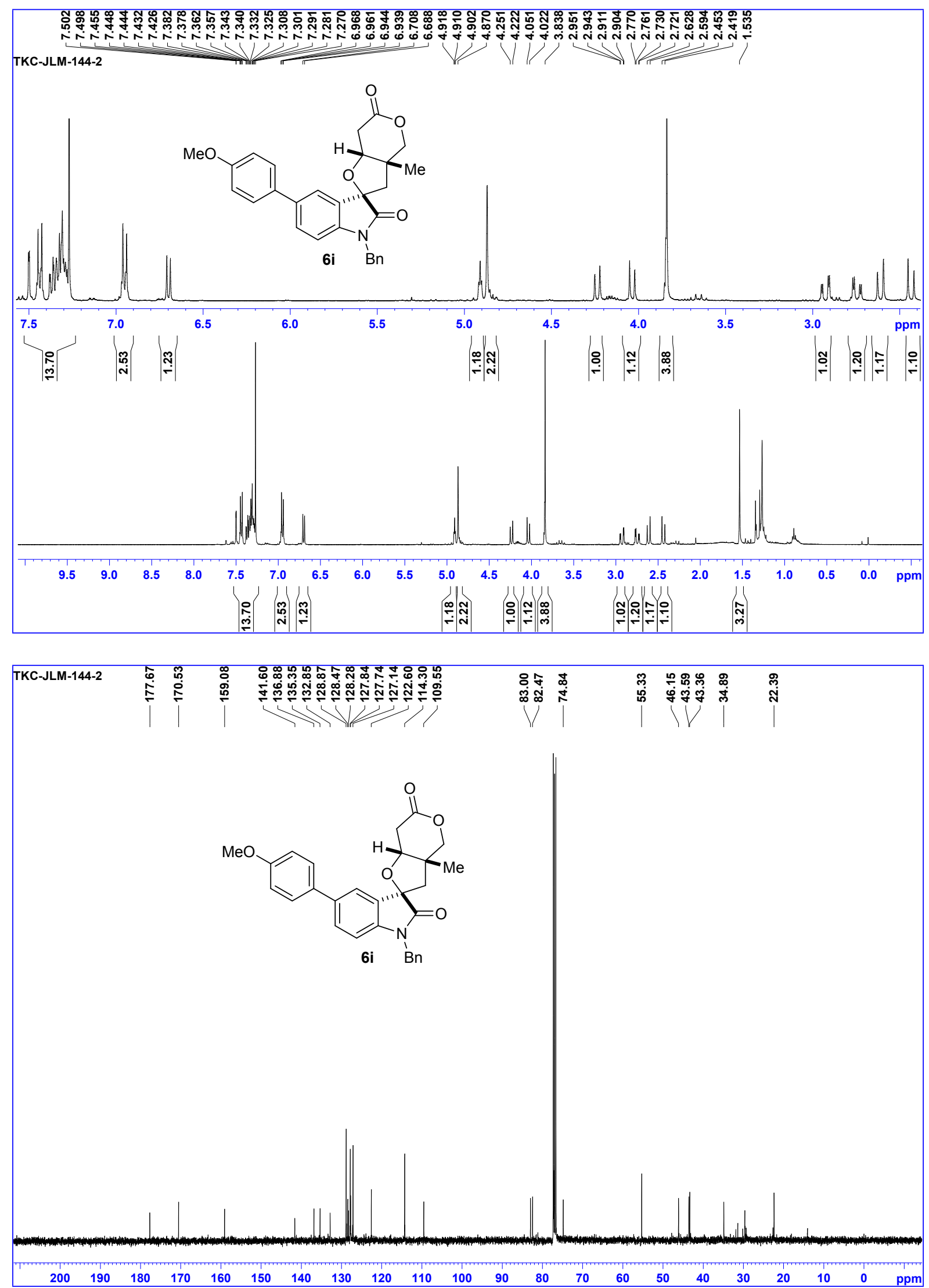
COSY and NOESY $\left(\mathrm{CDCl}_{3}, 400 \mathrm{MHz}\right)$ spectra of compound $\mathbf{6 i}$
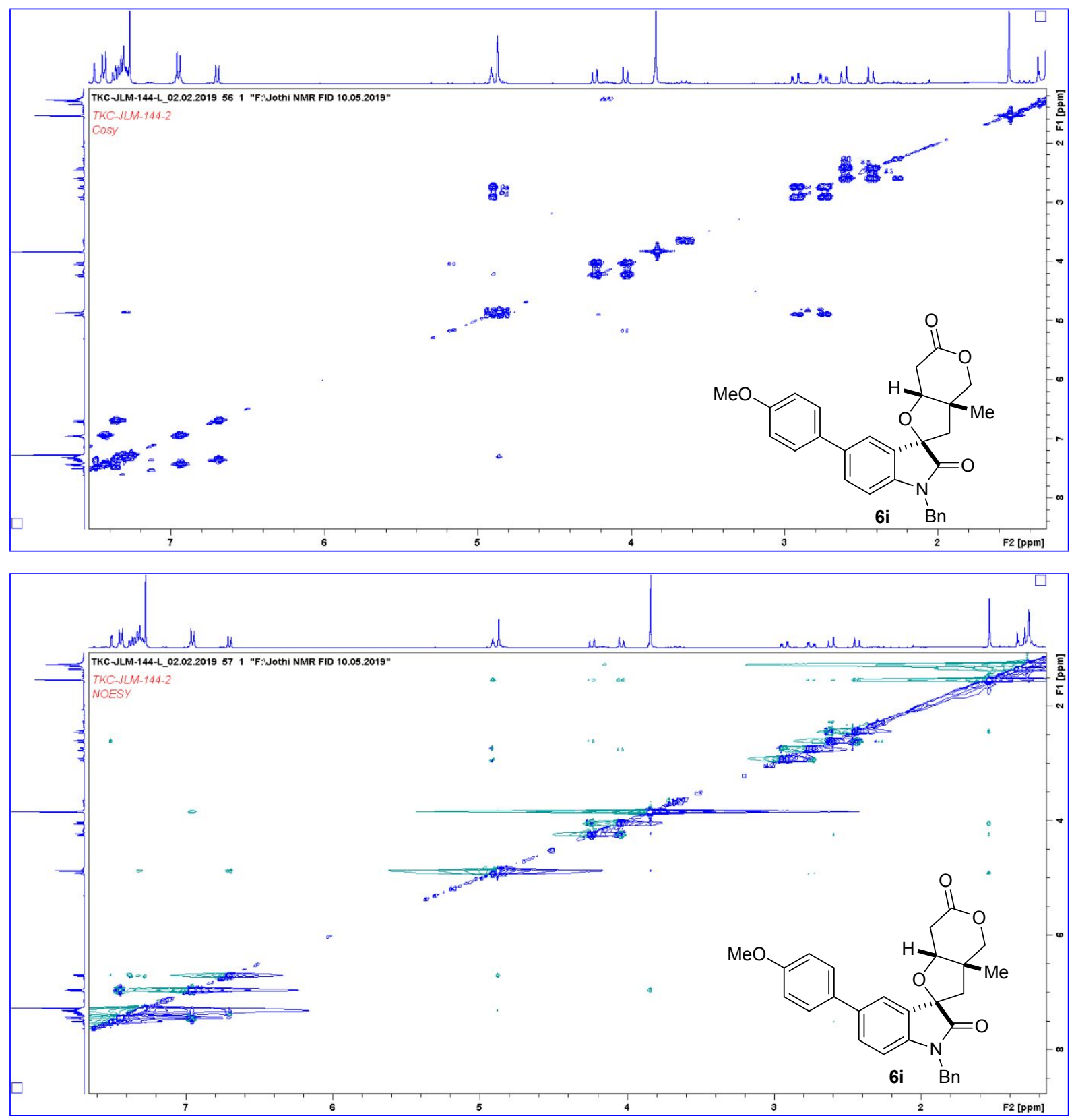
NOE $\left(\mathrm{CDCl}_{3}, 400 \mathrm{MHz}\right)$ spectra of compound $\mathbf{6 i}$
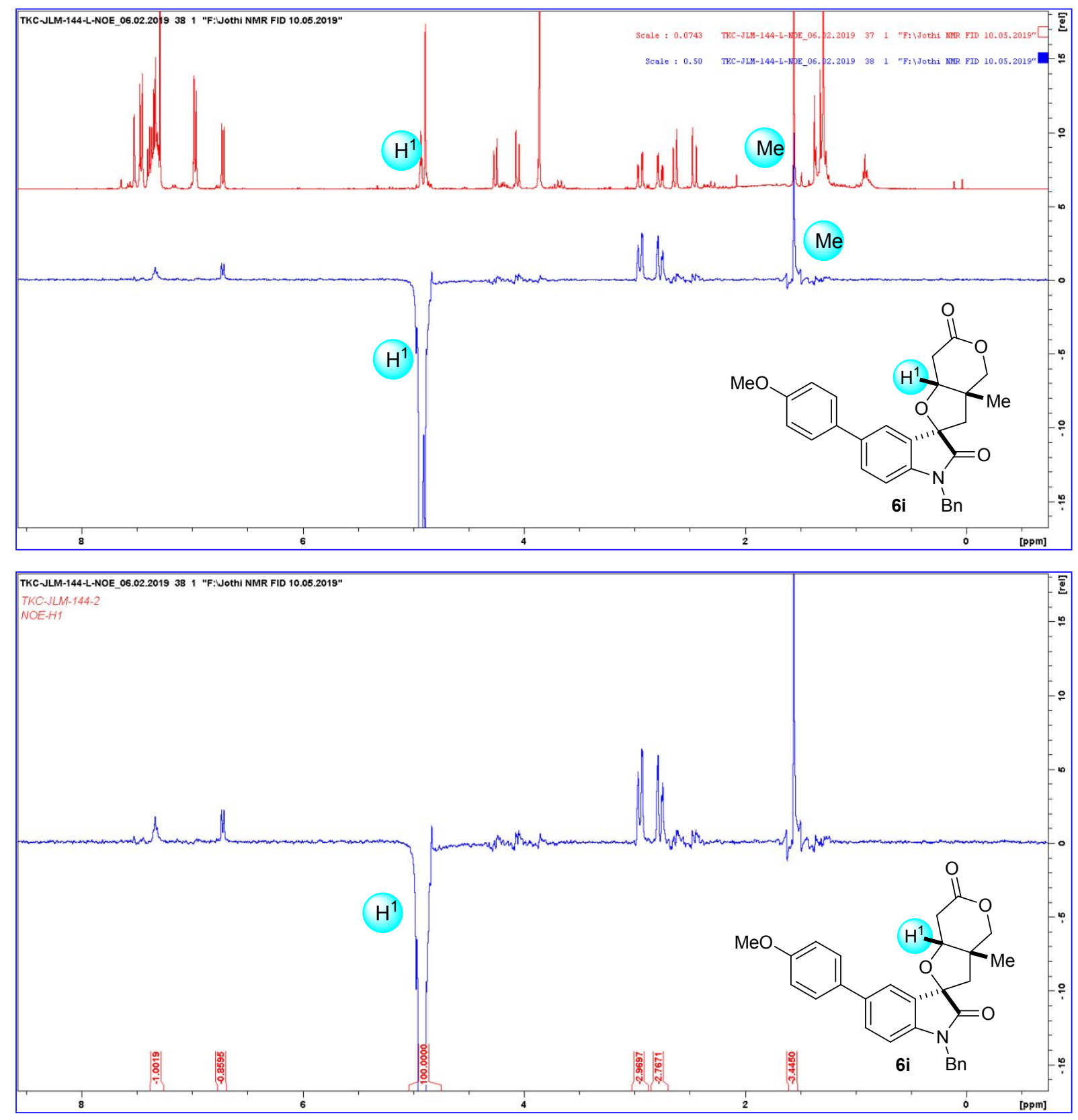
$\mathrm{HSQC}$ and $\mathrm{HMBC}\left(\mathrm{CDCl}_{3}, 400 \mathrm{MHz}\right)$ spectra of compound $\mathbf{6 i}$
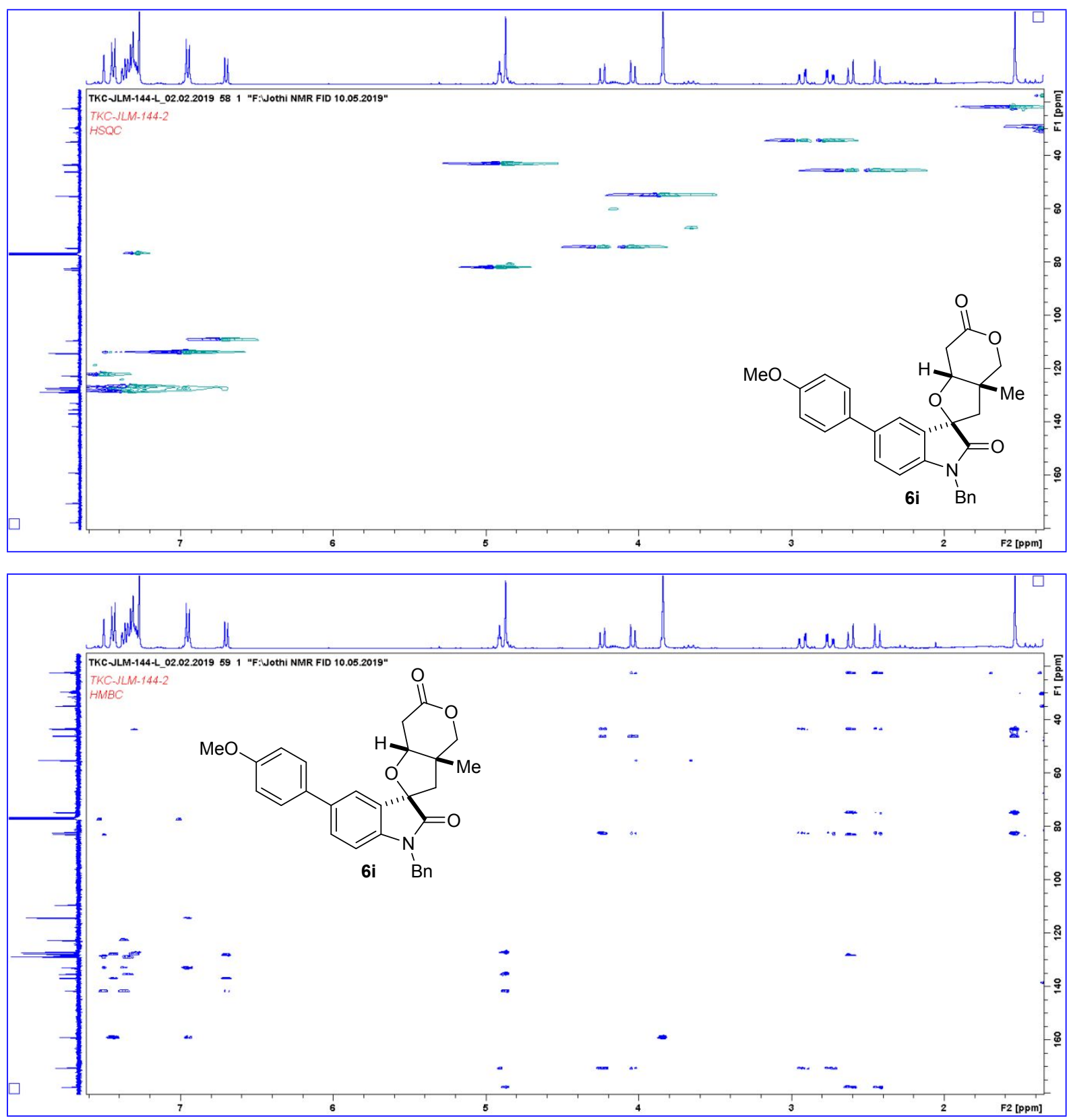
${ }^{1} \mathrm{H}-\mathrm{NMR}\left(\mathrm{CDCl}_{3}, 400 \mathrm{MHz}\right)$ and ${ }^{13} \mathrm{C}-\mathrm{NMR}\left(\mathrm{CDCl}_{3}, 100 \mathrm{MHz}\right)$ spectra of compound $\mathbf{6 i} \mathbf{i}^{\prime}$

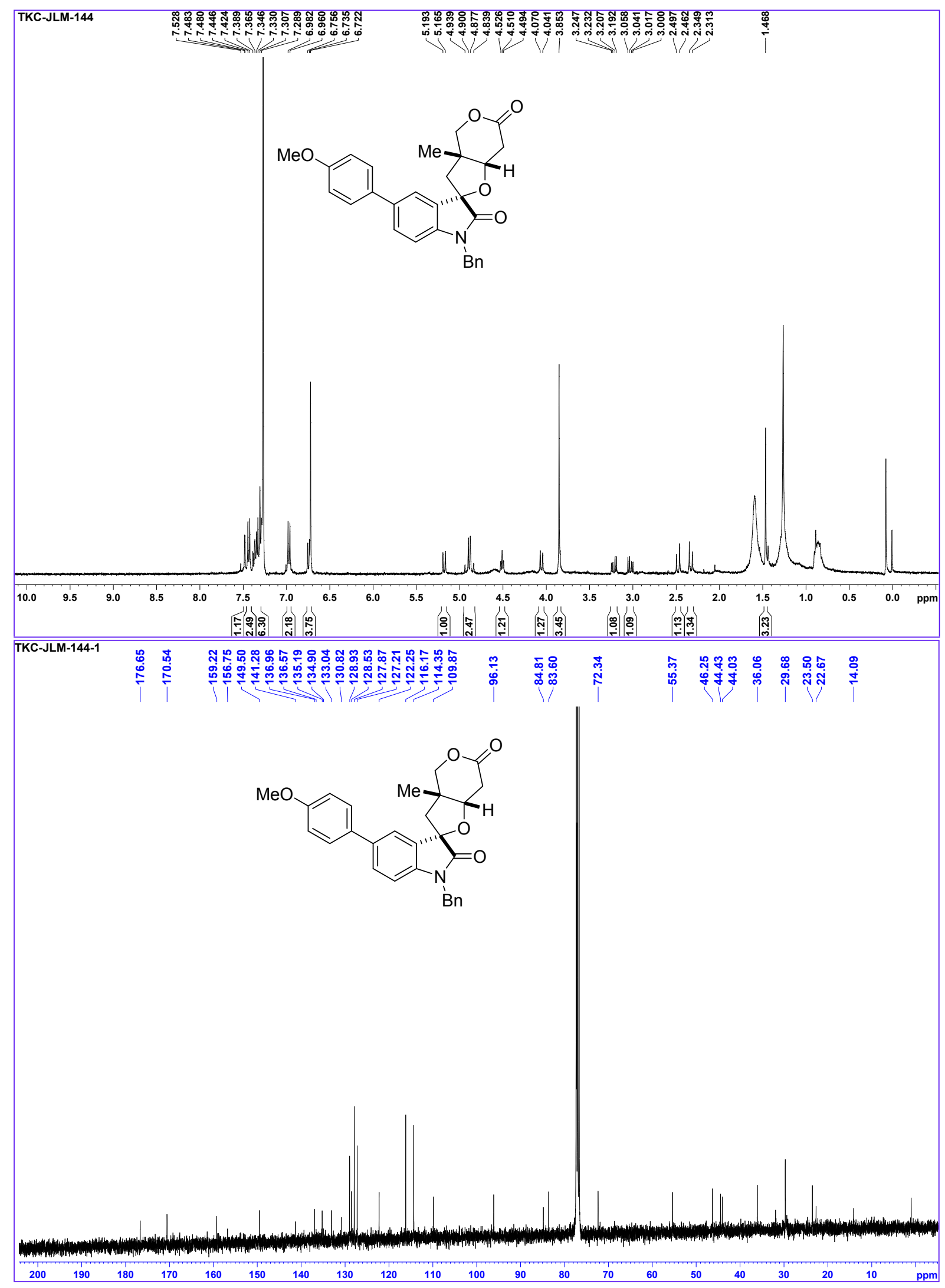


COSY and NOESY $\left(\mathrm{CDCl}_{3}, 400 \mathrm{MHz}\right)$ spectra of compound $\mathbf{6} \mathbf{i}^{\prime}$
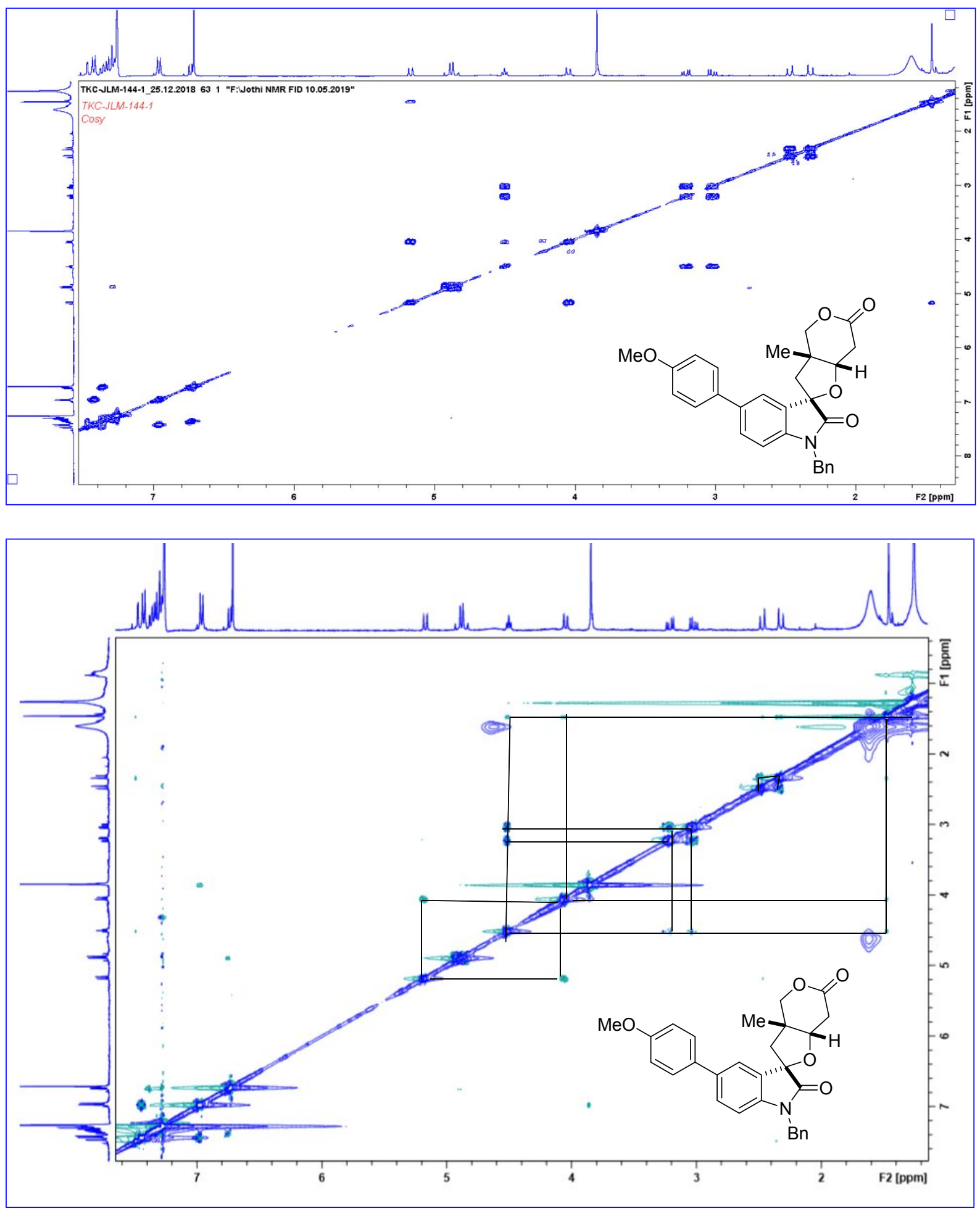
HMBC and $\mathrm{HSQC}\left(\mathrm{CDCl}_{3}, 400 \mathrm{MHz}\right)$ spectra of compound $6 \mathbf{i}^{\prime}$

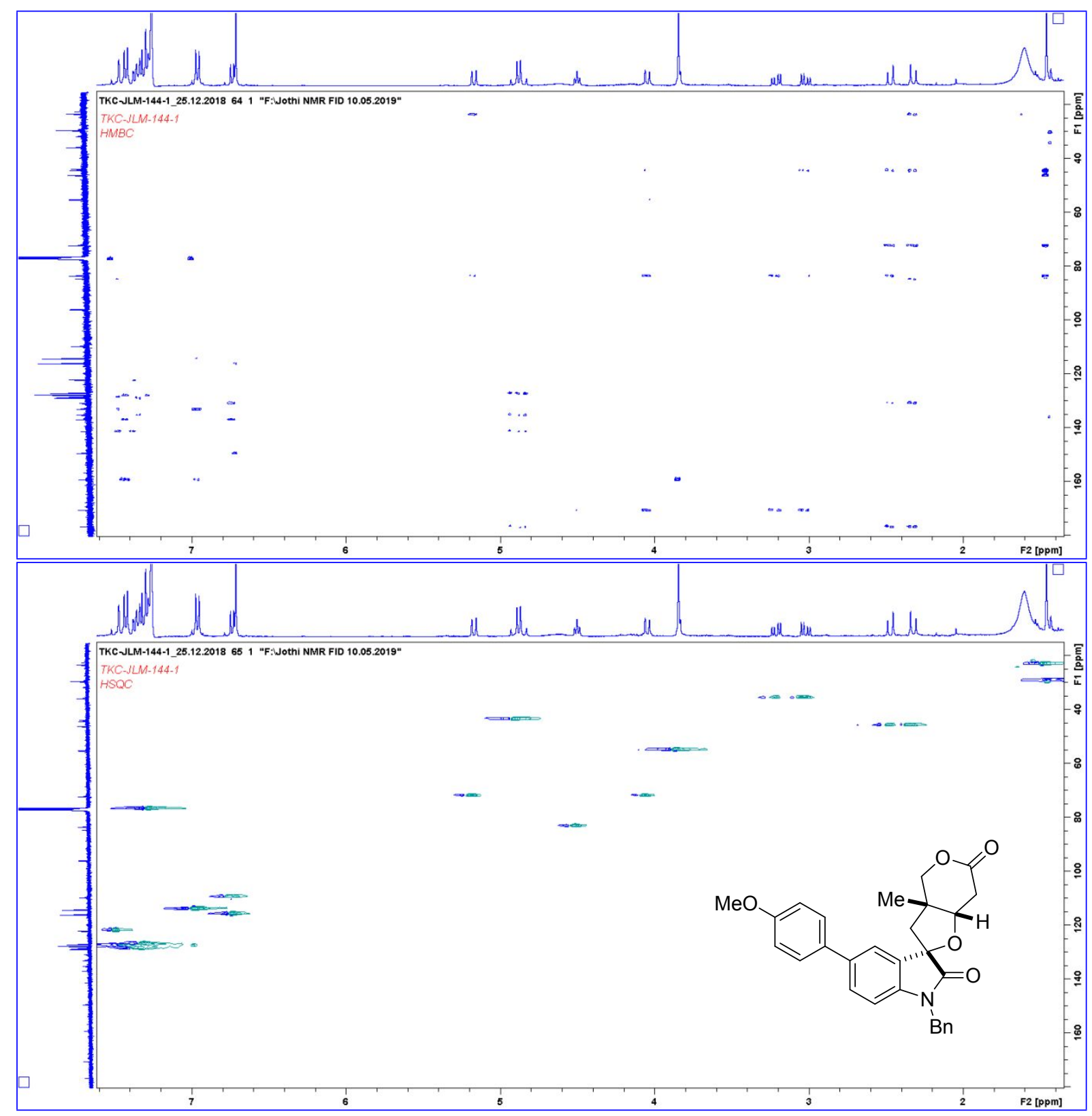


${ }^{1} \mathrm{H}-\mathrm{NMR}\left(\mathrm{CDCl}_{3}, 400 \mathrm{MHz}\right)$ and ${ }^{13} \mathrm{C}-\mathrm{NMR}\left(\mathrm{CDCl}_{3}, 100 \mathrm{MHz}\right)$ spectra of compound $\mathbf{5 j}$
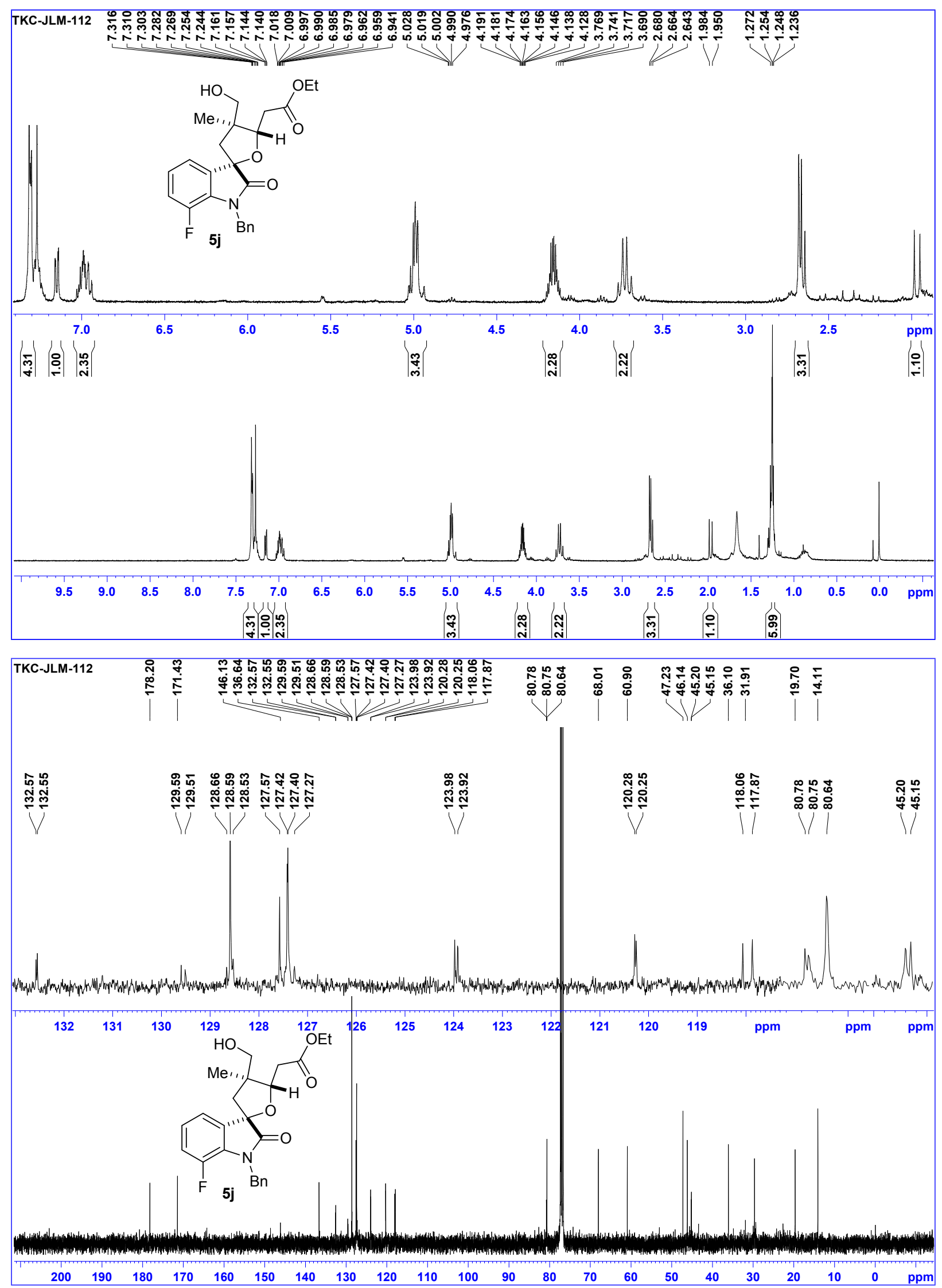
${ }^{1} \mathrm{H}-\mathrm{NMR}\left(\mathrm{CDCl}_{3}, 400 \mathrm{MHz}\right)$ spectrum of compound $\mathbf{6 j}$

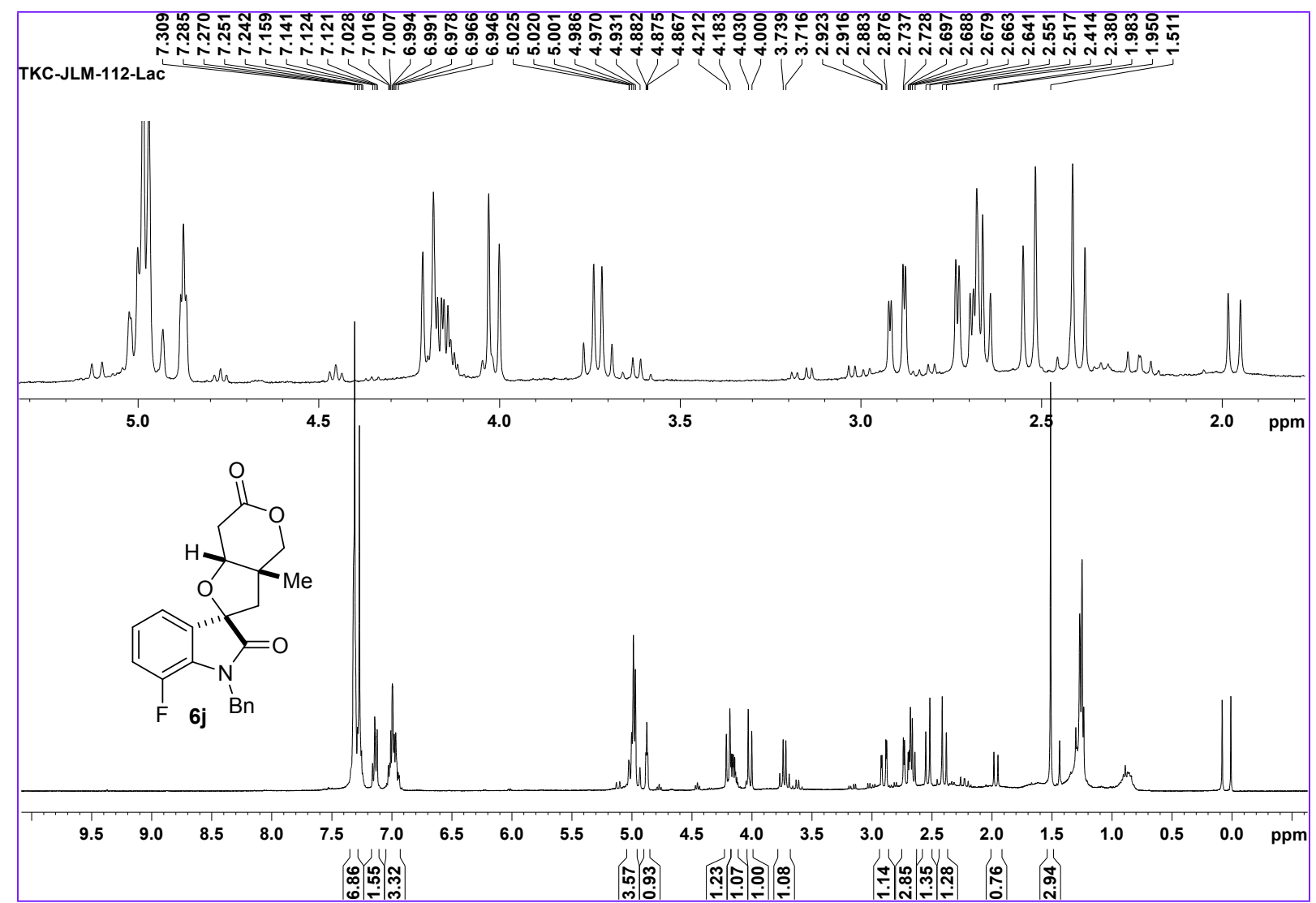


${ }^{1} \mathrm{H}-\mathrm{NMR}\left(\mathrm{CDCl}_{3}, 400 \mathrm{MHz}\right)$ and ${ }^{13} \mathrm{C}-\mathrm{NMR}\left(\mathrm{CDCl}_{3}, 100 \mathrm{MHz}\right)$ spectra of compound $7 \mathbf{a}$
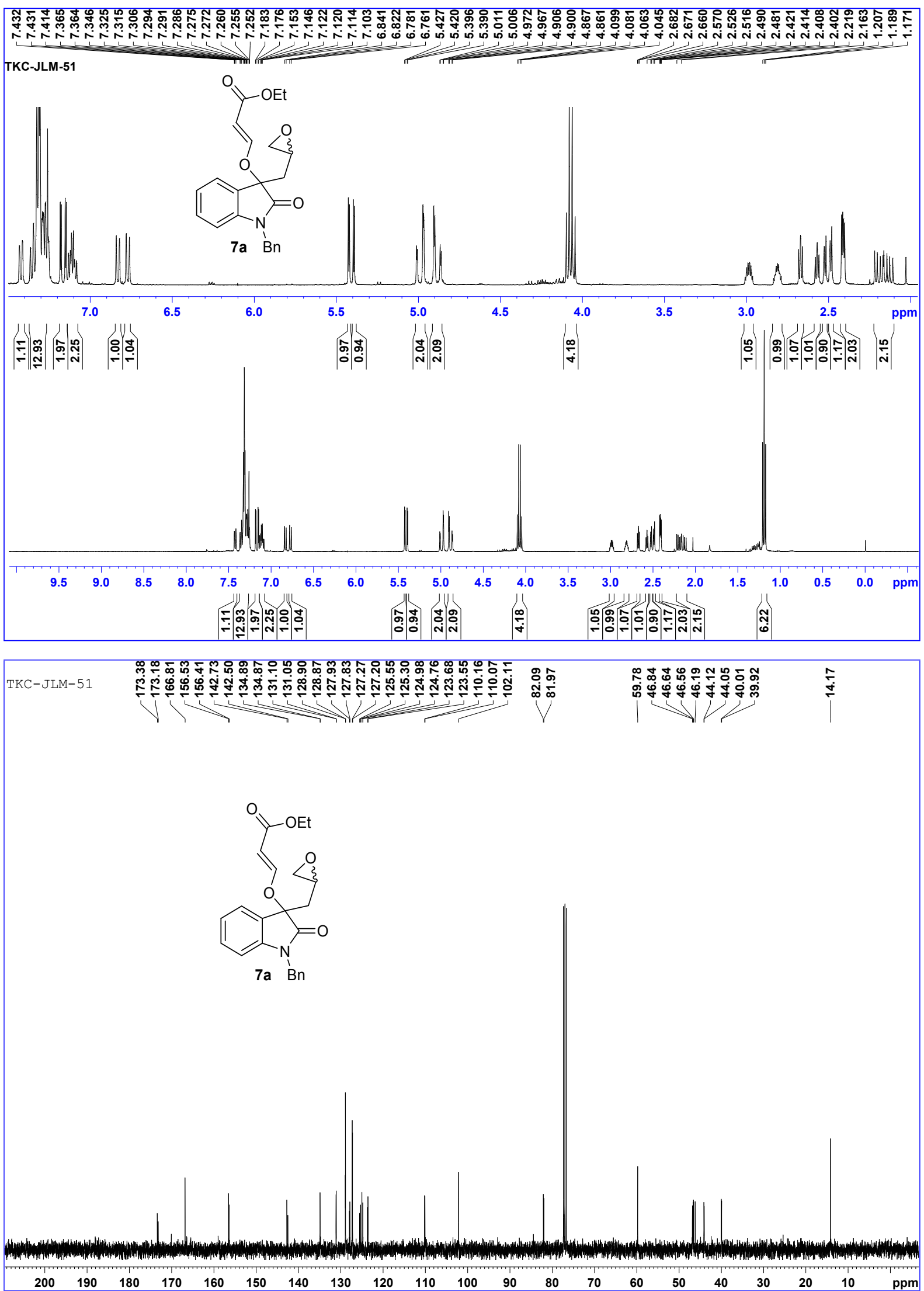
${ }^{1} \mathrm{H}-\mathrm{NMR}\left(\mathrm{CDCl}_{3}, 400 \mathrm{MHz}\right)$ and ${ }^{13} \mathrm{C}-\mathrm{NMR}\left(\mathrm{CDCl}_{3}, 100 \mathrm{MHz}\right)$ spectra of compound $7 \mathbf{b}$
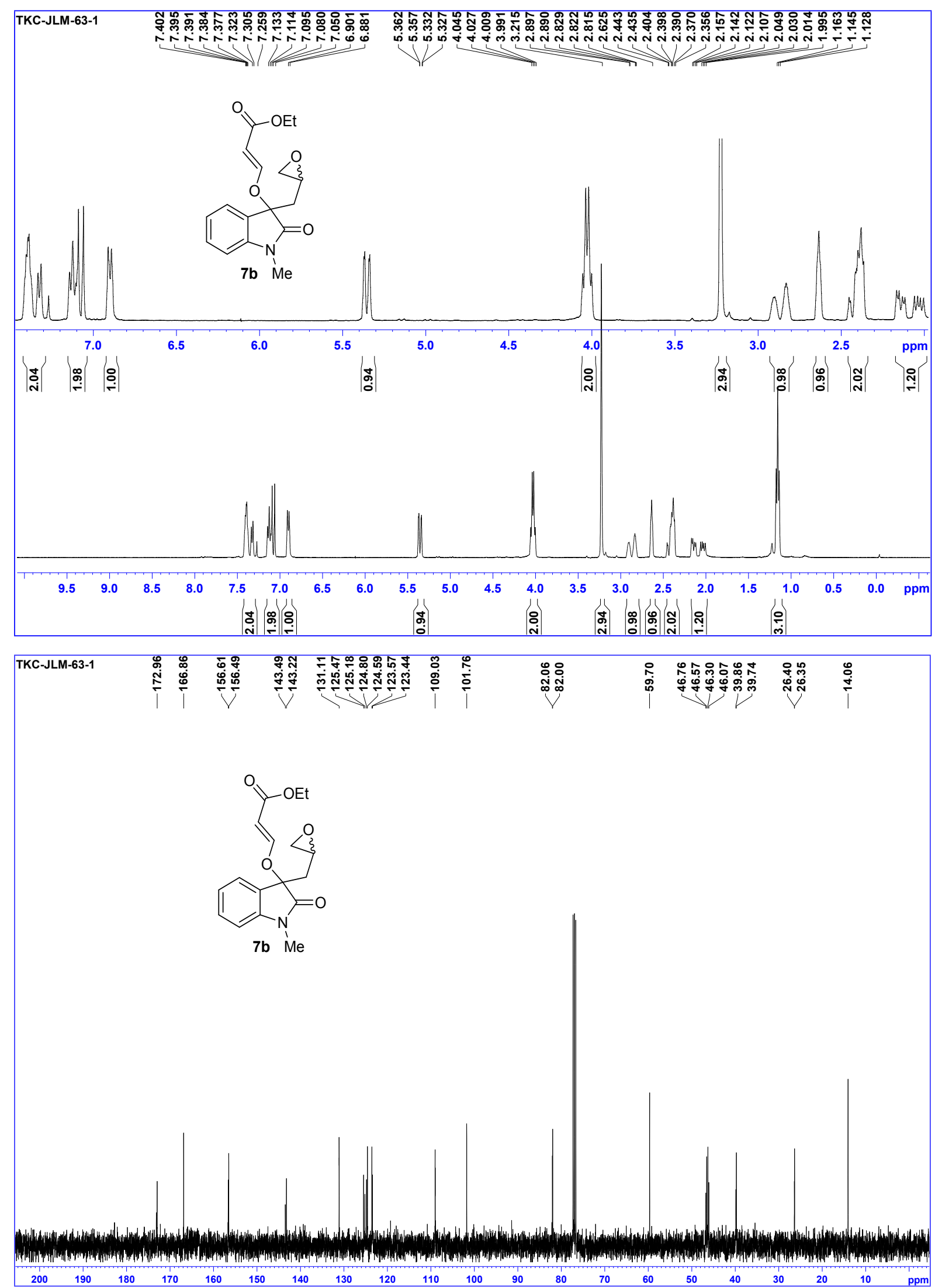
${ }^{1} \mathrm{H}-\mathrm{NMR}\left(\mathrm{CDCl}_{3}, 400 \mathrm{MHz}\right)$ and ${ }^{13} \mathrm{C}-\mathrm{NMR}\left(\mathrm{CDCl}_{3}, 100 \mathrm{MHz}\right)$ spectra of compound 7c
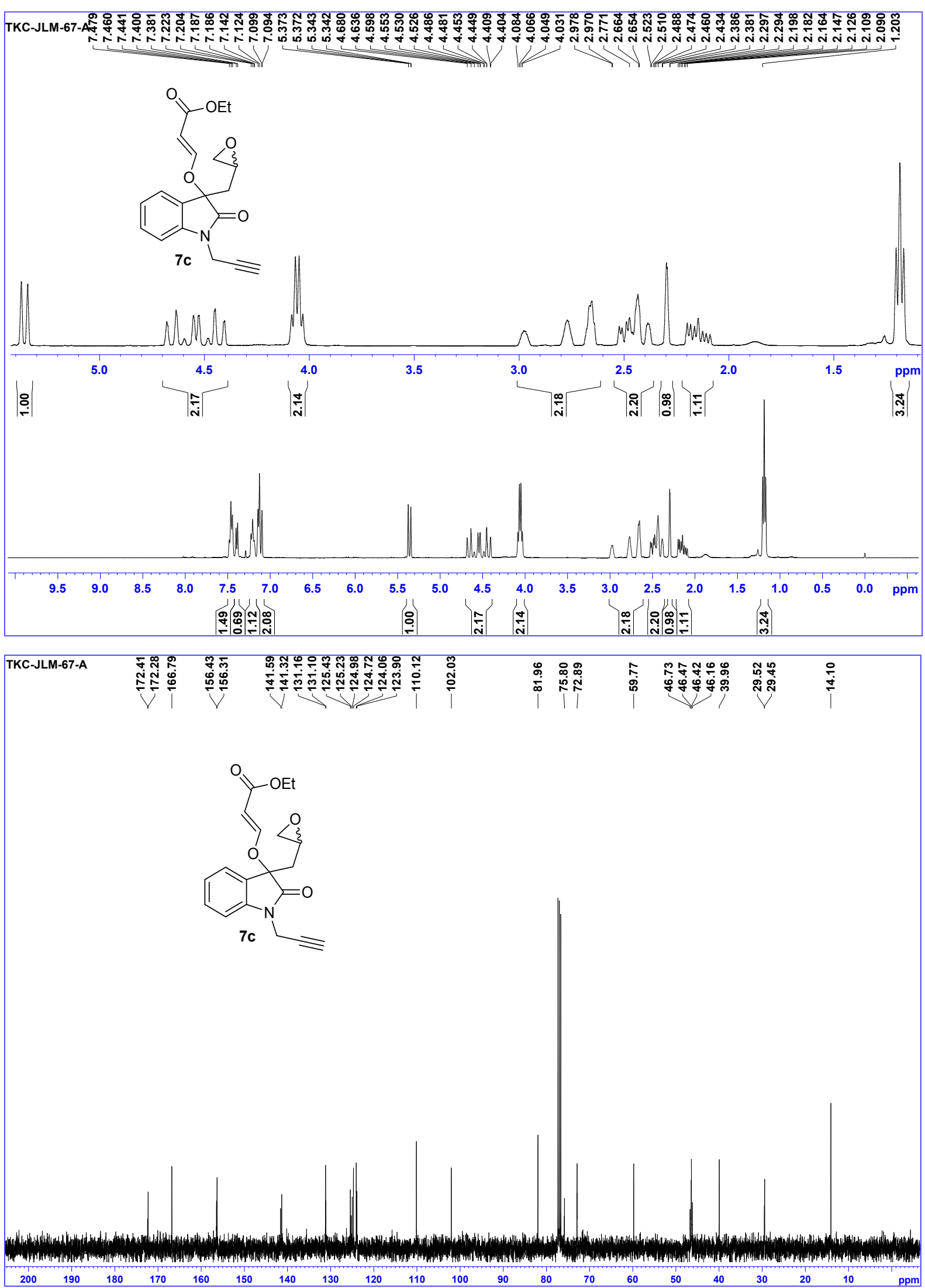
${ }^{1} \mathrm{H}-\mathrm{NMR}\left(\mathrm{CDCl}_{3}, 400 \mathrm{MHz}\right)$ and ${ }^{13} \mathrm{C}-\mathrm{NMR}\left(\mathrm{CDCl}_{3}, 100 \mathrm{MHz}\right)$ spectra of compound $7 \mathbf{d}$
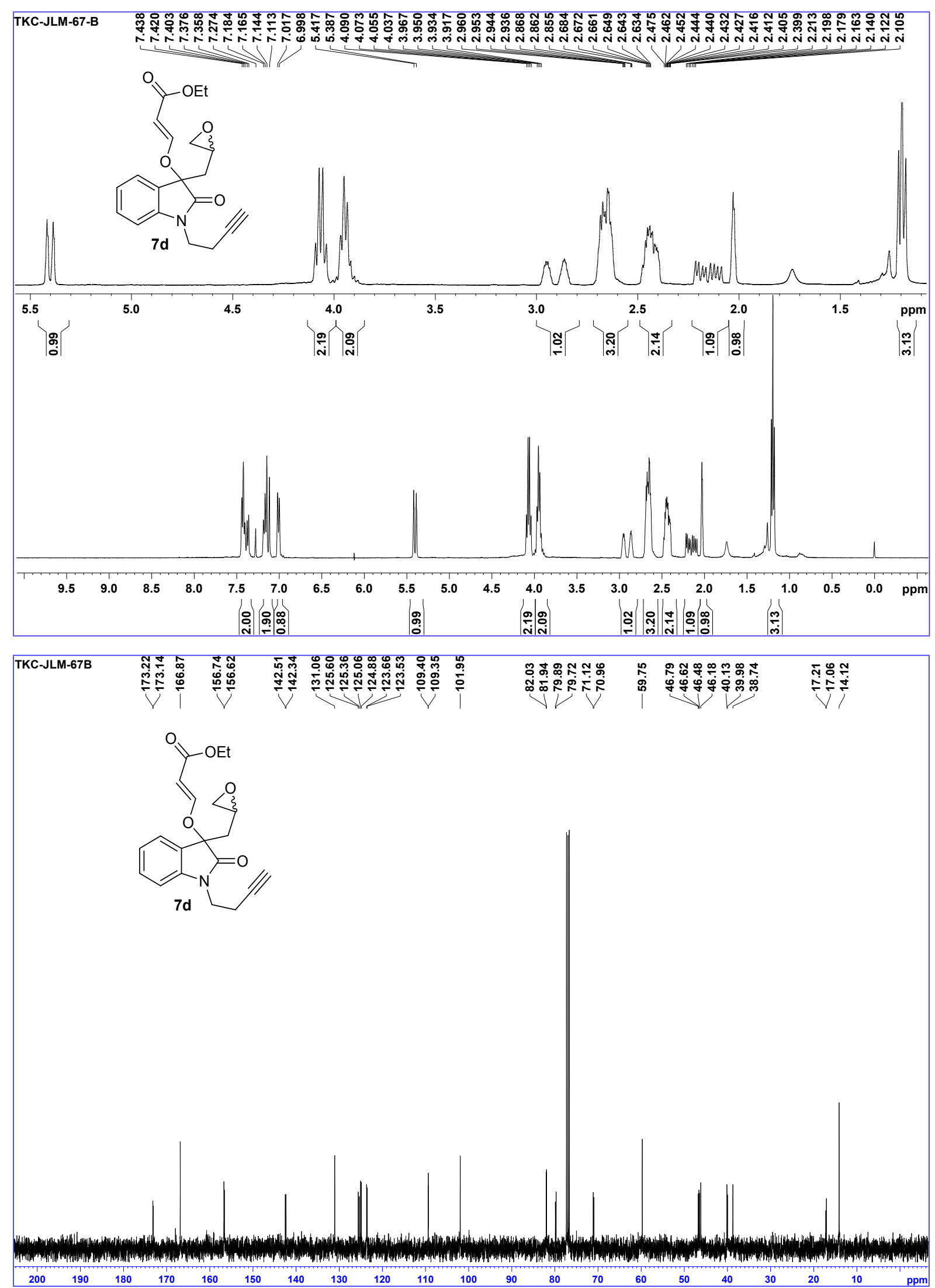
${ }^{1} \mathrm{H}-\mathrm{NMR}\left(\mathrm{CD}_{3} \mathrm{OD}, 400 \mathrm{MHz}\right)$ and ${ }^{13} \mathrm{C}-\mathrm{NMR}\left(\mathrm{CD}_{3} \mathrm{OD}, 100 \mathrm{MHz}\right)$ spectra of compound 8a
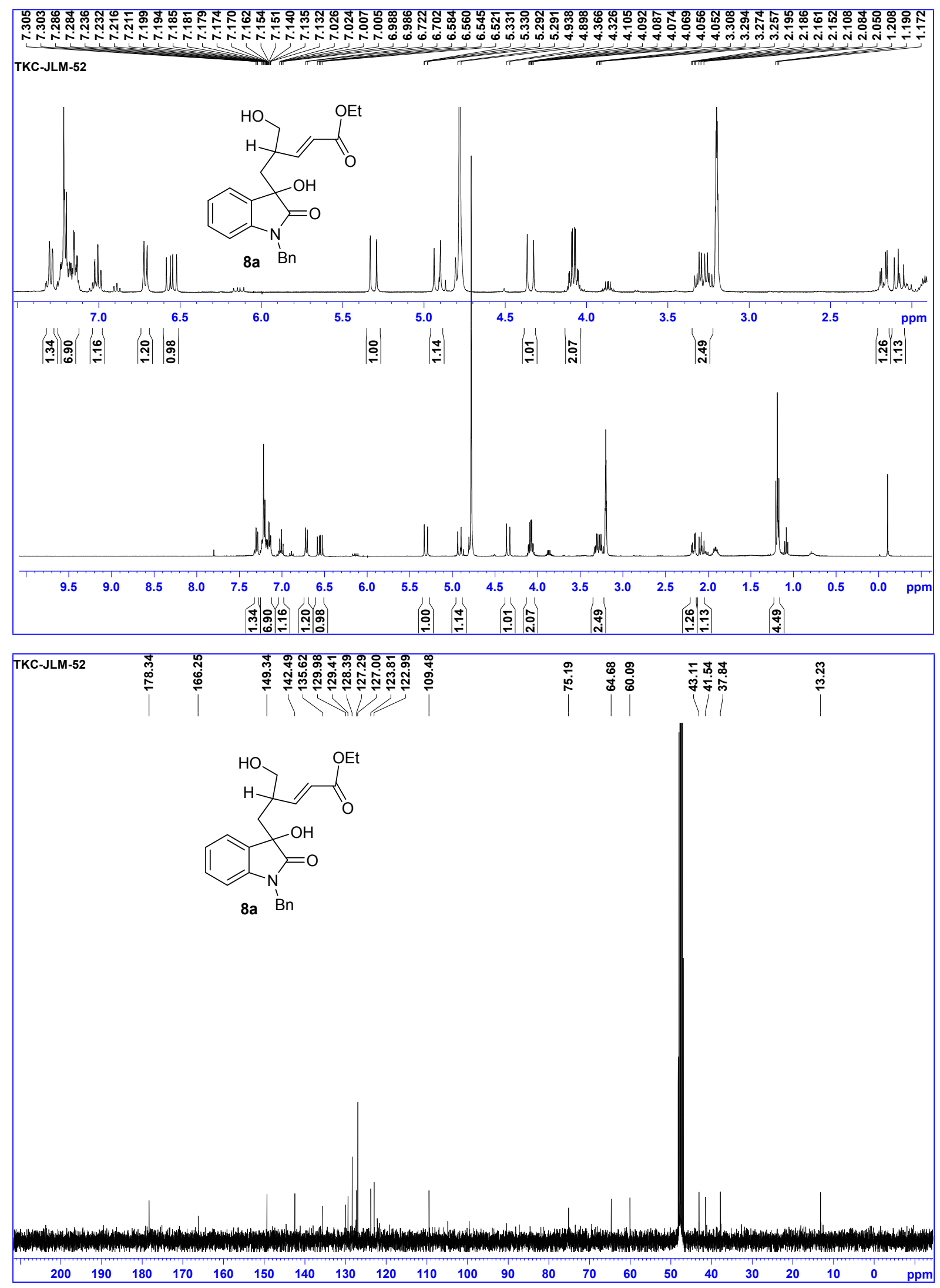
${ }^{1} \mathrm{H}-\mathrm{NMR}\left(\mathrm{CDCl}_{3}, 400 \mathrm{MHz}\right)$ spectra of compound 9a

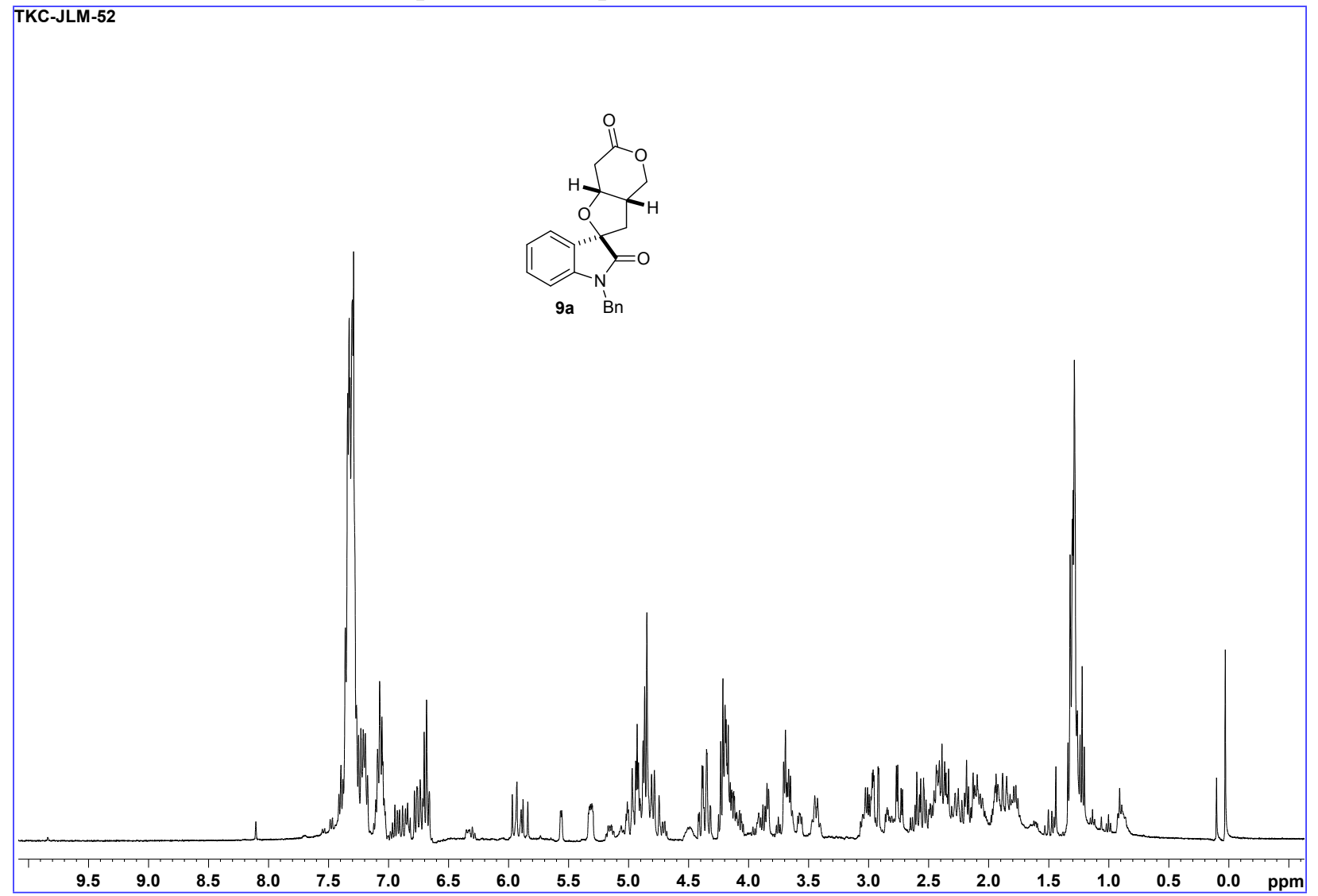


${ }^{1} \mathrm{H}-\mathrm{NMR}\left(\mathrm{CDCl}_{3}, 400 \mathrm{MHz}\right)$ and ${ }^{13} \mathrm{C}-\mathrm{NMR}\left(\mathrm{CDCl}_{3}, 100 \mathrm{MHz}\right)$ spectra of compound $\mathbf{8 b}$
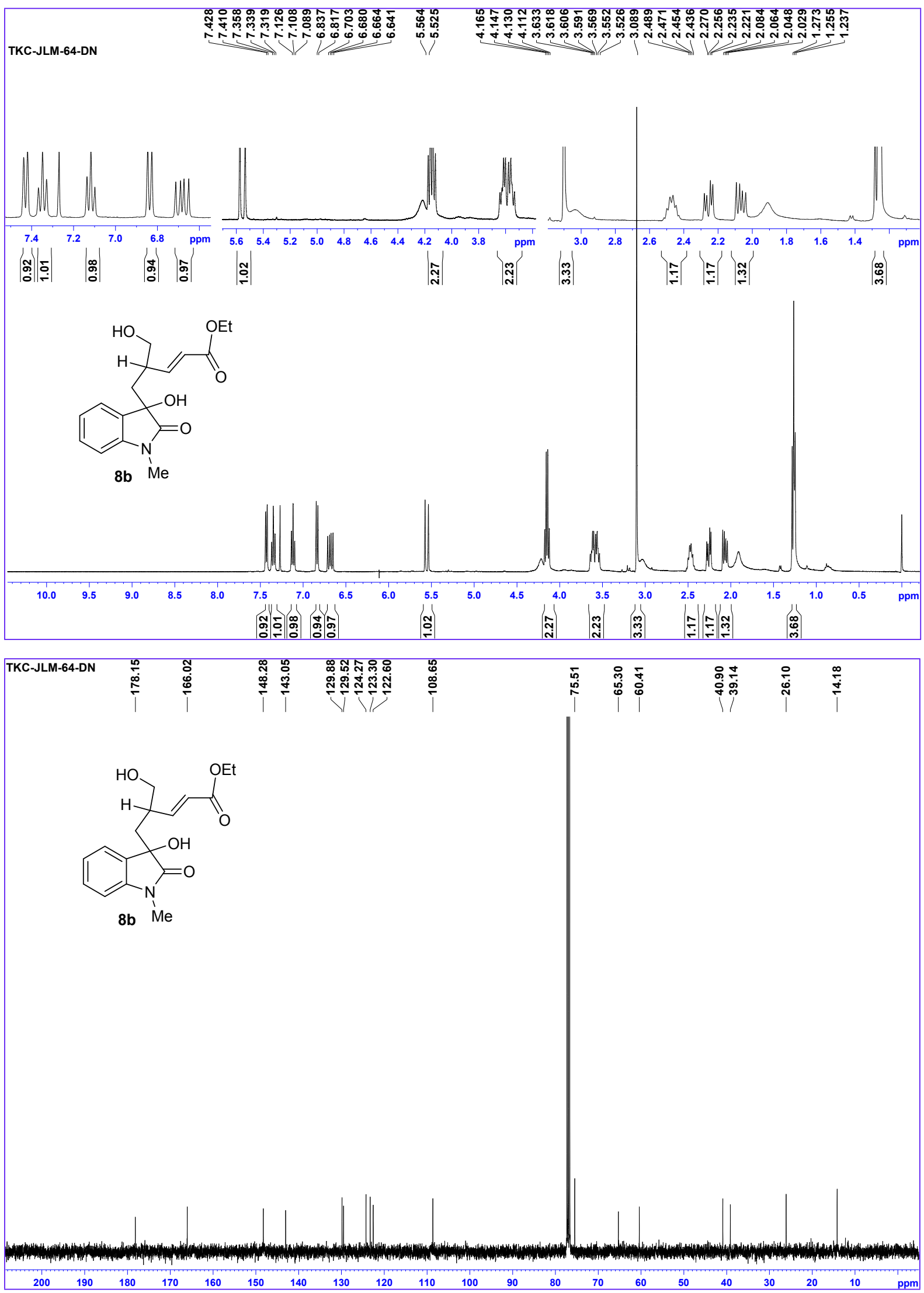
${ }^{1} \mathrm{H}-\mathrm{NMR}\left(\mathrm{CDCl}_{3}, 400 \mathrm{MHz}\right)$ spectra of compound $\mathbf{9 b}$

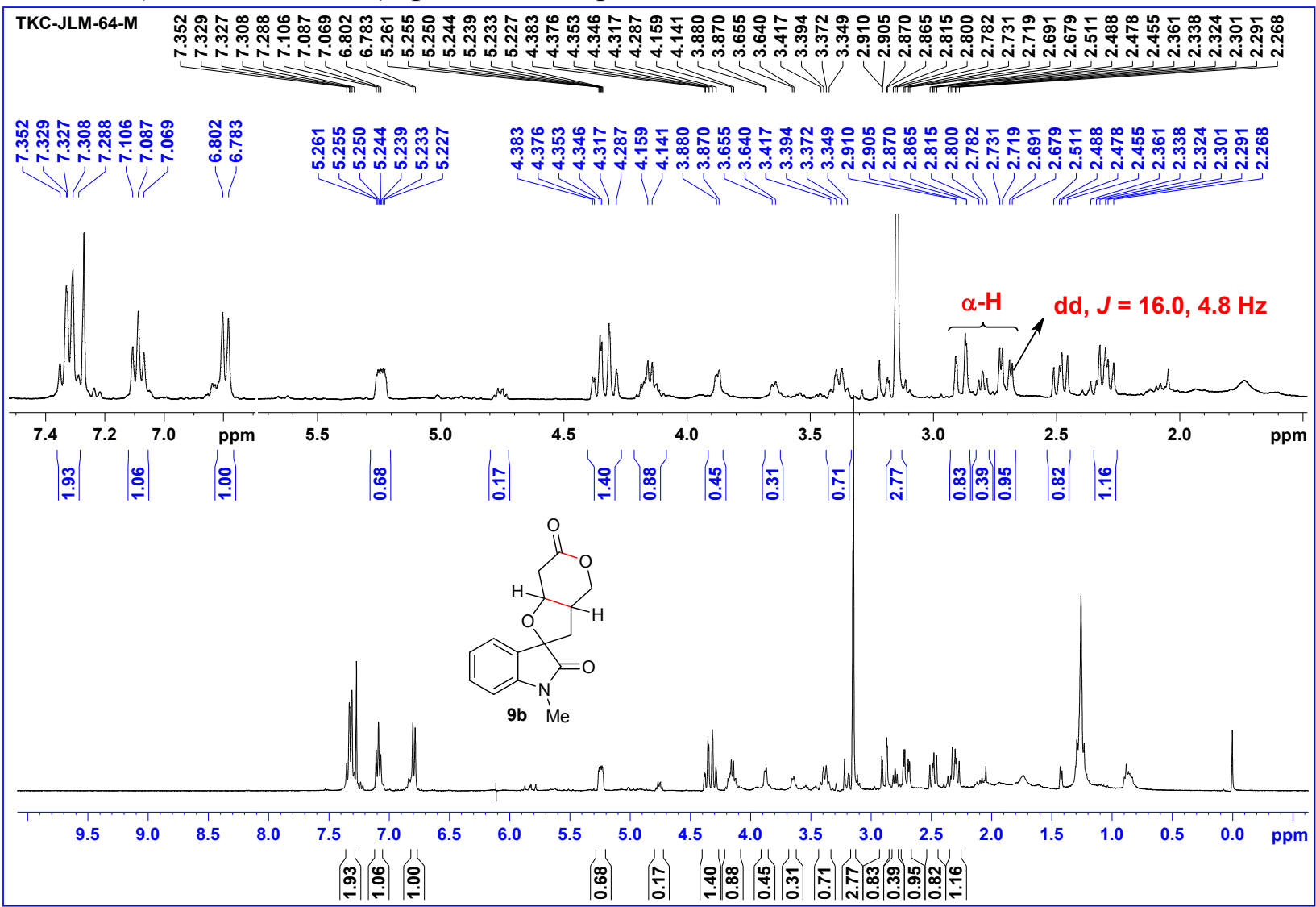


${ }^{1} \mathrm{H}-\mathrm{NMR}\left(\mathrm{CDCl}_{3}, 400 \mathrm{MHz}\right)$ and ${ }^{13} \mathrm{C}-\mathrm{NMR}\left(\mathrm{CDCl}_{3}, 100 \mathrm{MHz}\right)$ spectra of compound $\mathbf{8 c}$

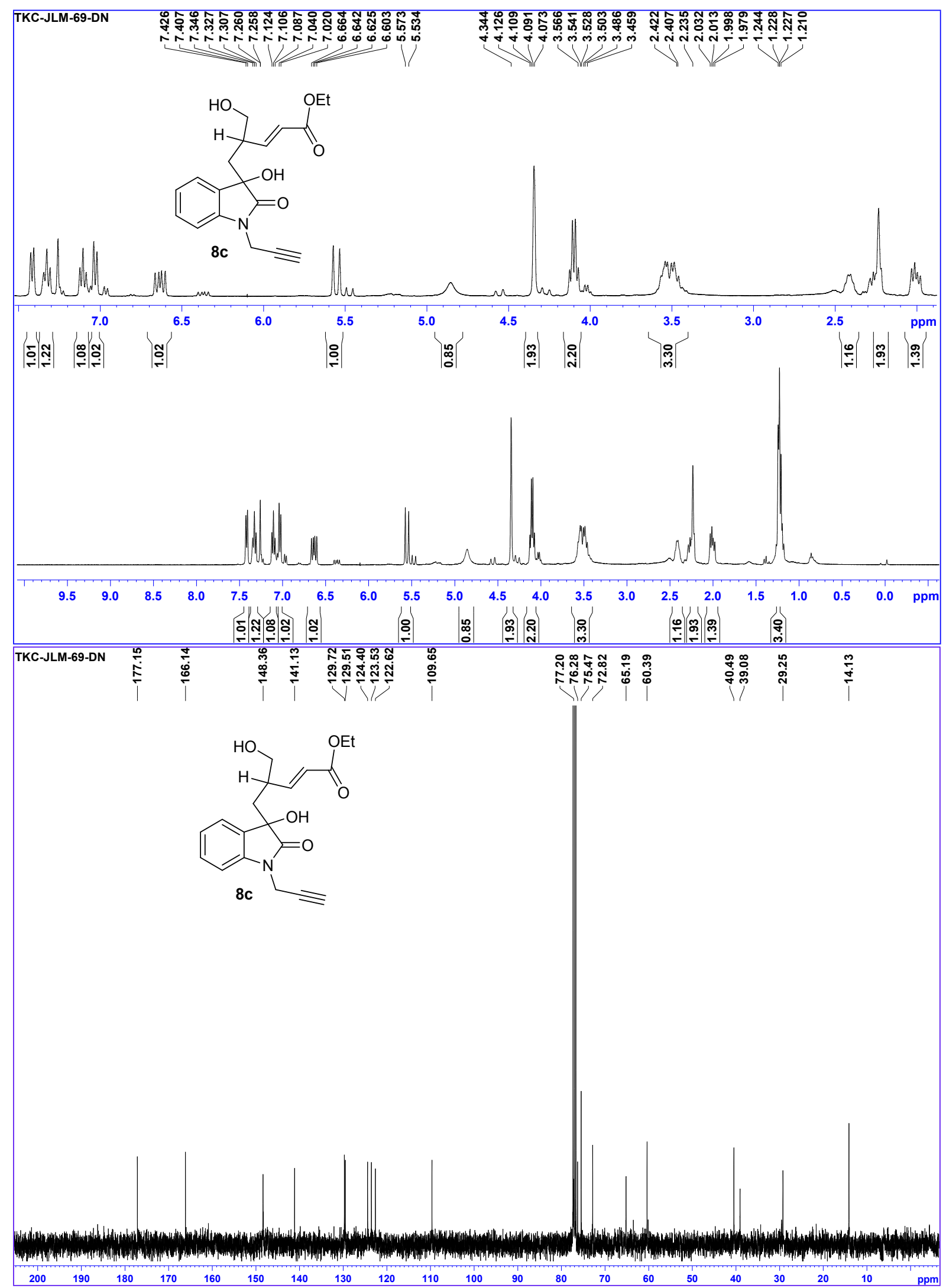


${ }^{1} \mathrm{H}-\mathrm{NMR}\left(\mathrm{CDCl}_{3}, 400 \mathrm{MHz}\right)$ spectra of compound 9c

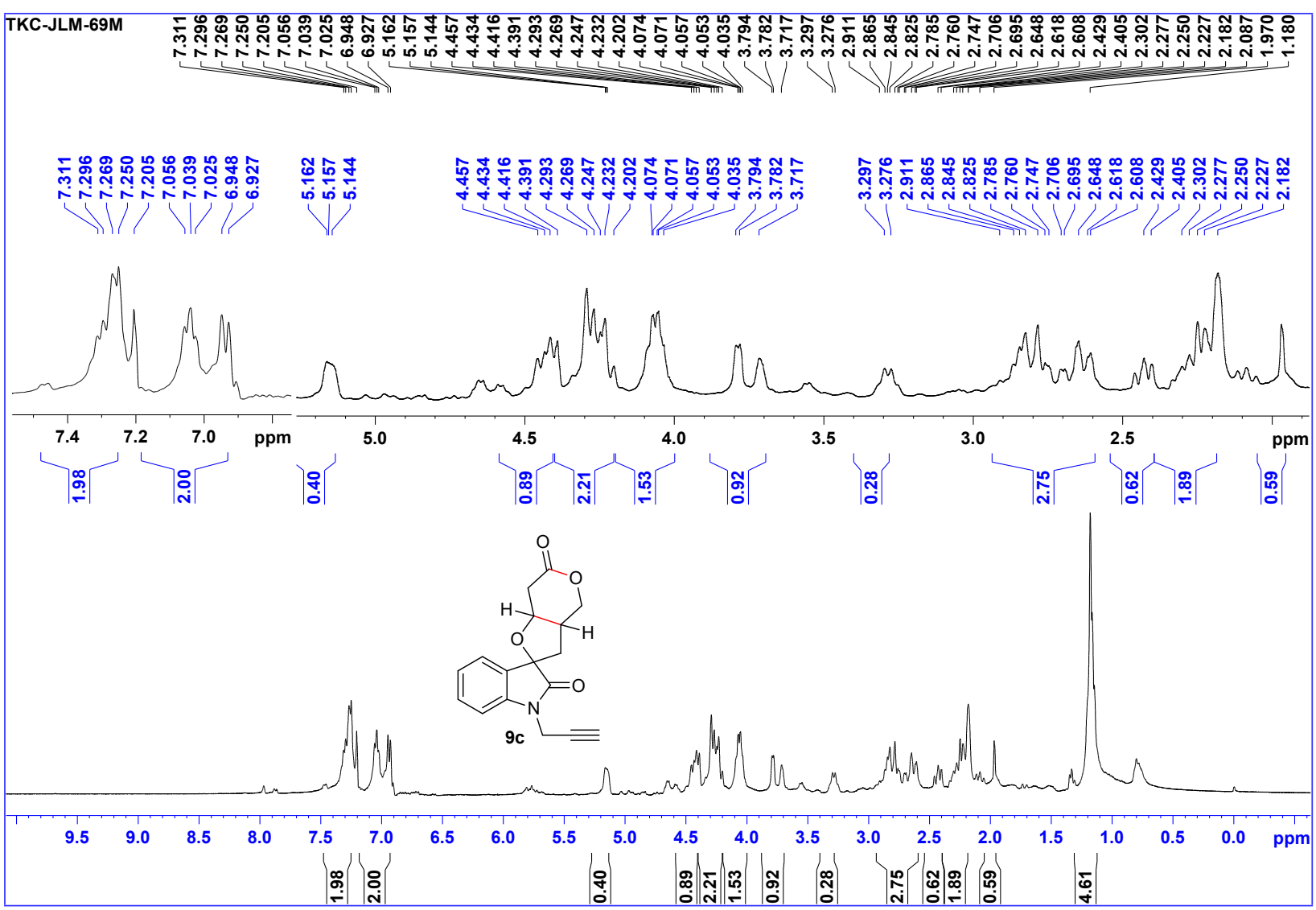


${ }^{1} \mathrm{H}-\mathrm{NMR}\left(\mathrm{CDCl}_{3}, 400 \mathrm{MHz}\right)$ and ${ }^{13} \mathrm{C}-\mathrm{NMR}\left(\mathrm{CDCl}_{3}, 100 \mathrm{MHz}\right)$ spectra of compound $8 \mathbf{d}$
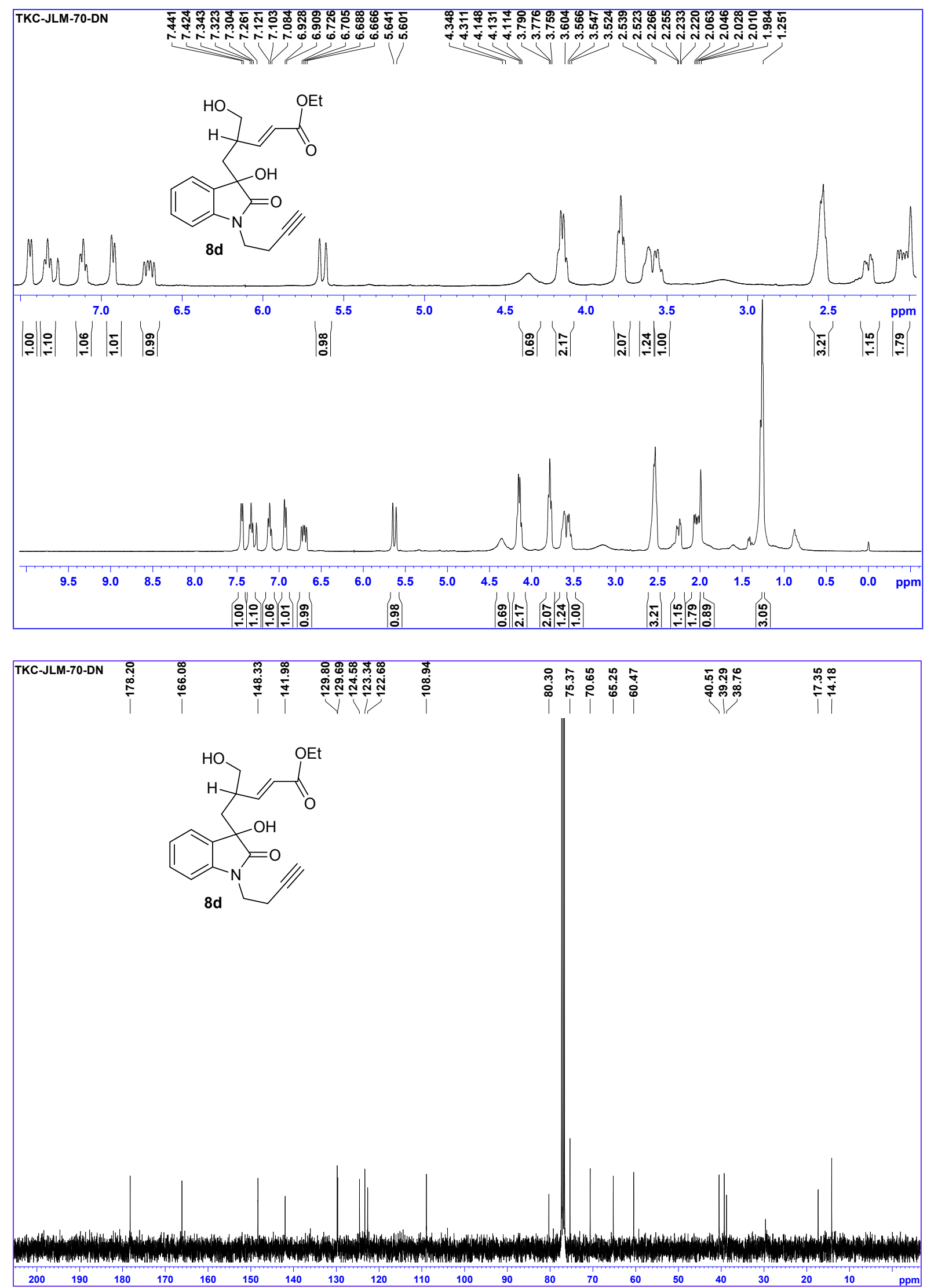
${ }^{1} \mathrm{H}-\mathrm{NMR}\left(\mathrm{CDCl}_{3}, 400 \mathrm{MHz}\right)$ spectra of compound 9d

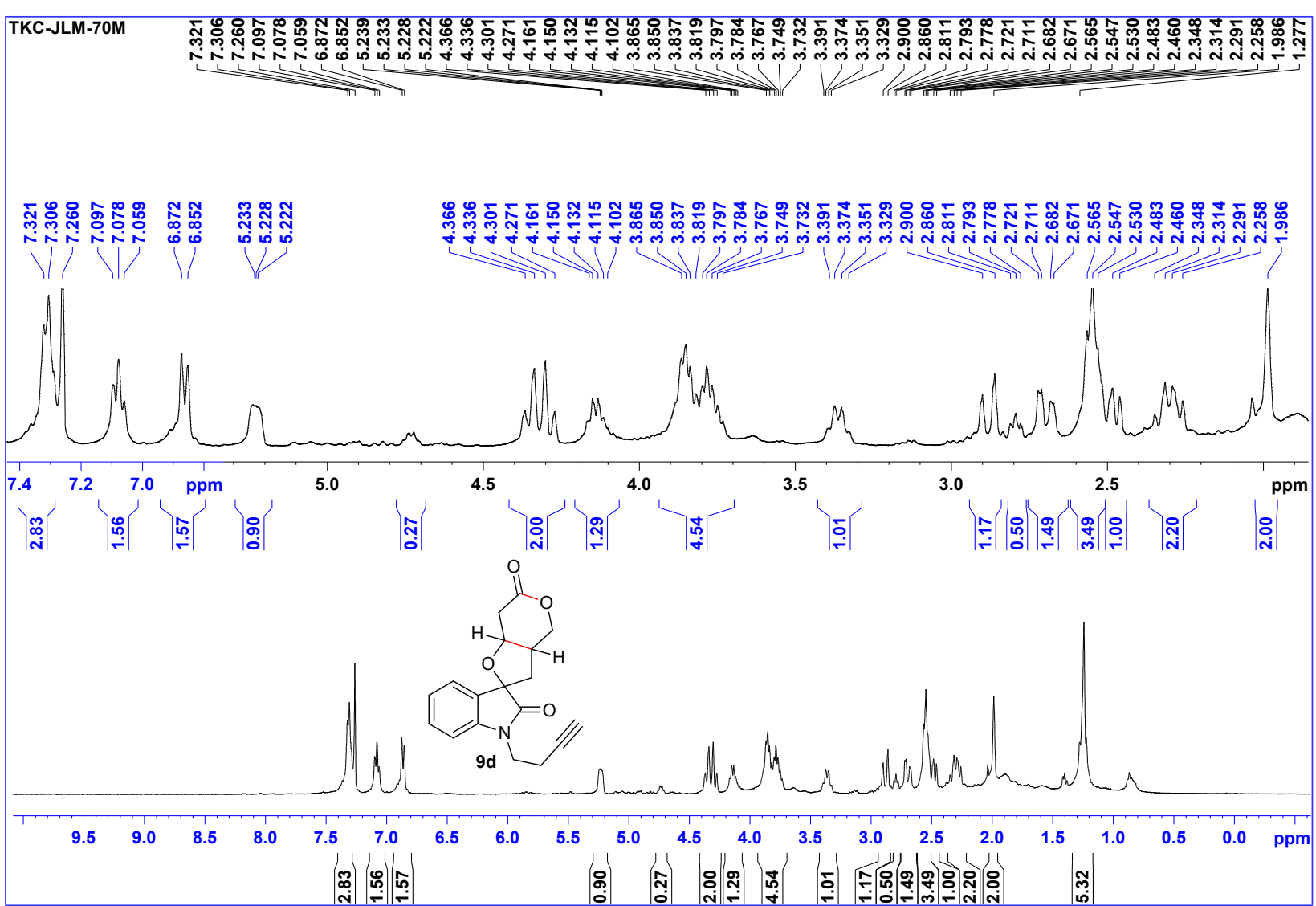


${ }^{1} \mathrm{H}-\mathrm{NMR}\left(\mathrm{CDCl}_{3}, 400 \mathrm{MHz}\right)$ and ${ }^{13} \mathrm{C}-\mathrm{NMR}\left(\mathrm{CDCl}_{3}, 100 \mathrm{MHz}\right)$ spectra of compound 10a
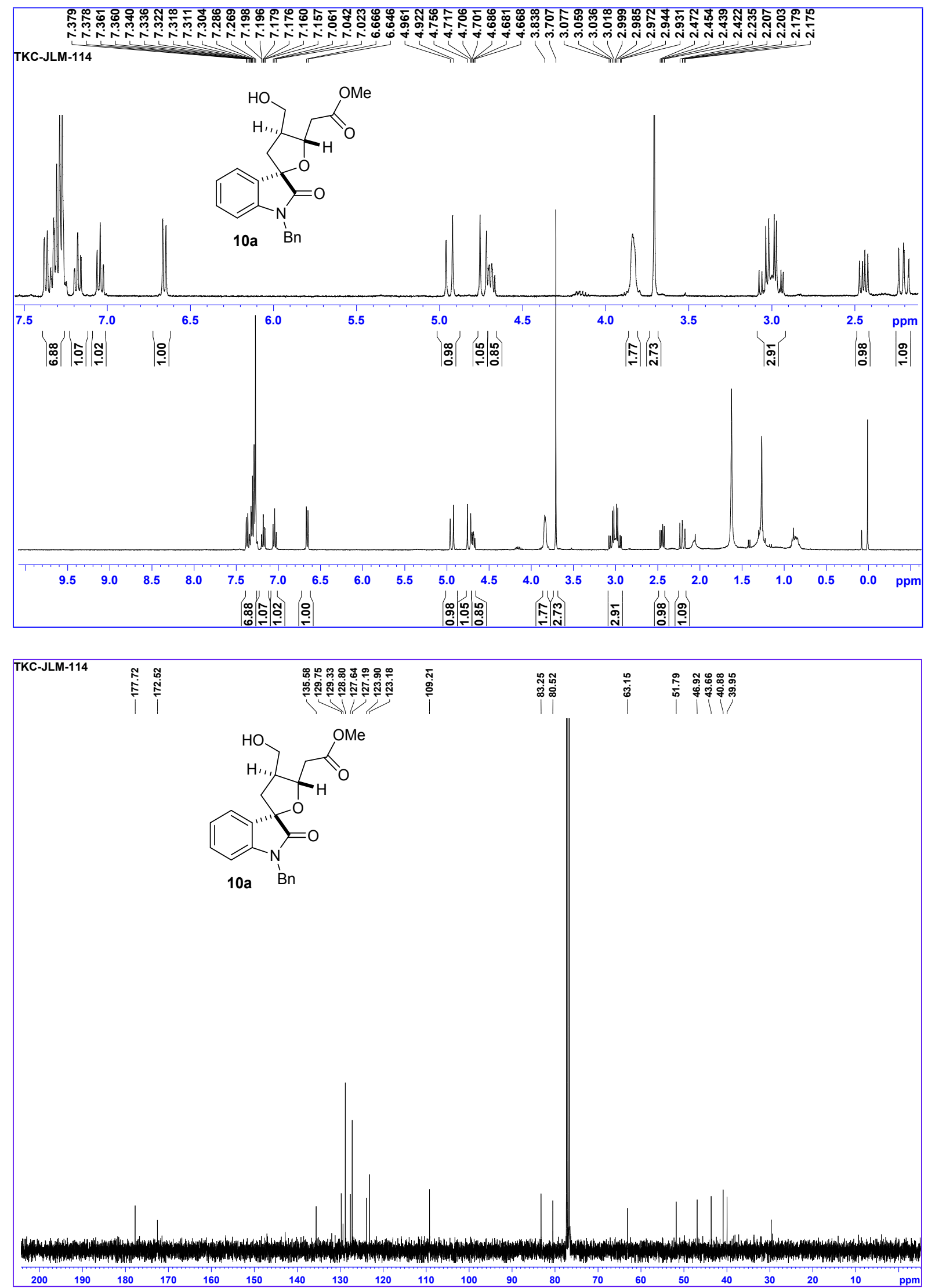
${ }^{1} \mathrm{H}-\mathrm{NMR}\left(\mathrm{CDCl}_{3}, 400 \mathrm{MHz}\right)$ and ${ }^{13} \mathrm{C}-\mathrm{NMR}\left(\mathrm{CDCl}_{3}, 100 \mathrm{MHz}\right)$ spectra of compound $\mathbf{1 0 b}$

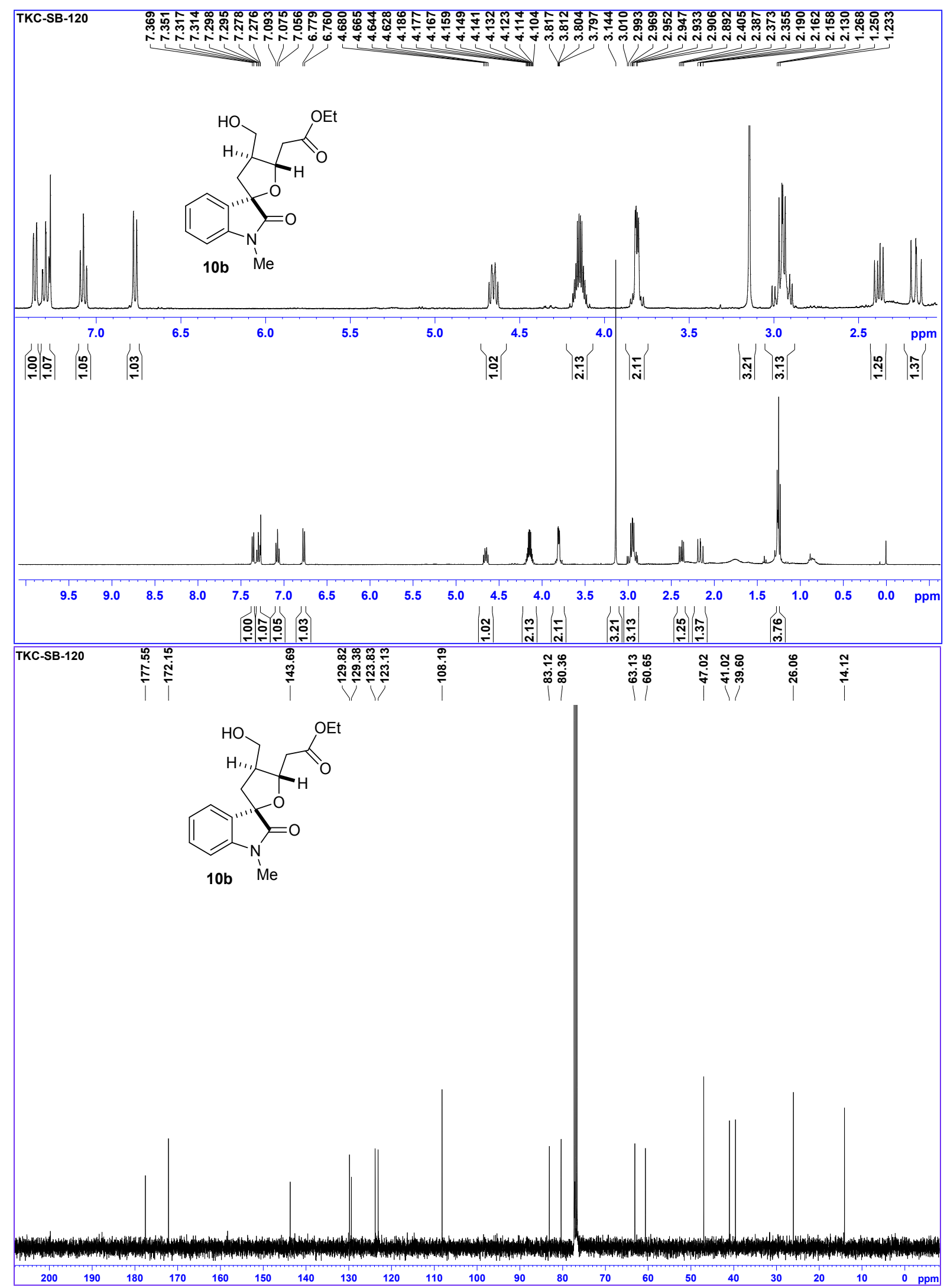


NOE and COSY $\left(\mathrm{CDCl}_{3}, 400 \mathrm{MHz}\right)$ spectra of compound 10b
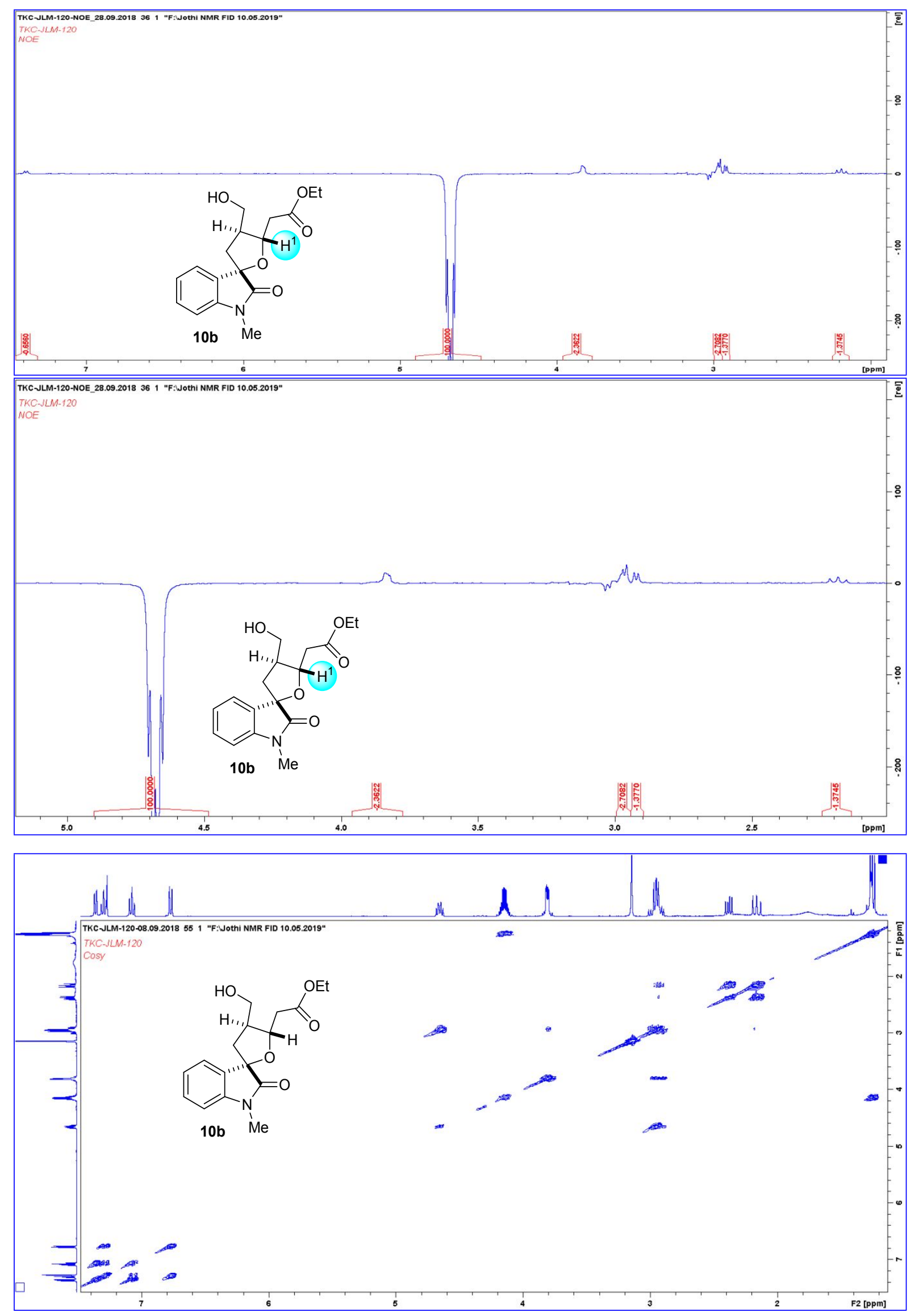
NOESY and HMQC $\left(\mathrm{CDCl}_{3}, 400 \mathrm{MHz}\right)$ spectra of compound 10b
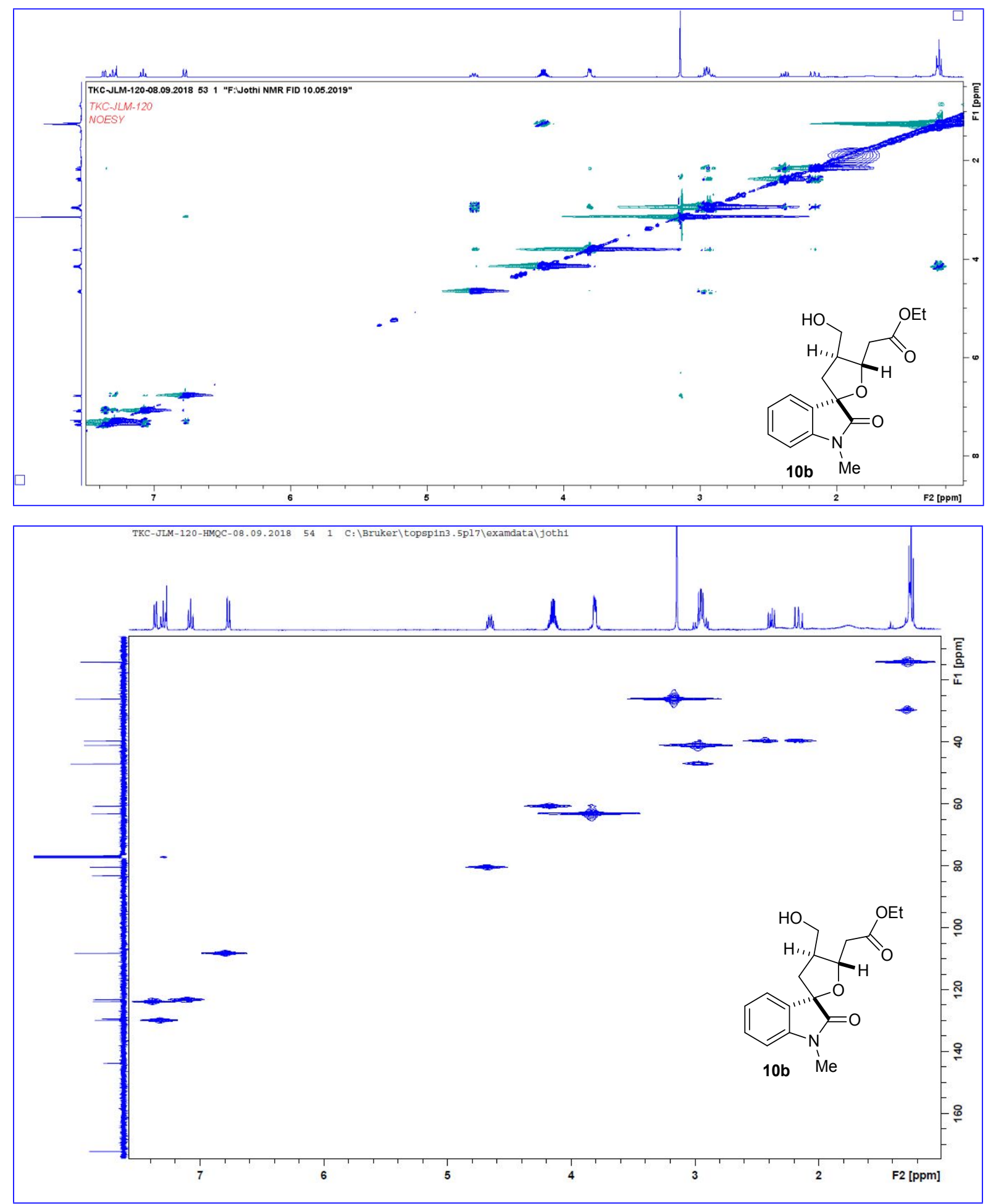
${ }^{1} \mathrm{H}-\mathrm{NMR}\left(\mathrm{CDCl}_{3}, 400 \mathrm{MHz}\right)$ and ${ }^{13} \mathrm{C}-\mathrm{NMR}\left(\mathrm{CDCl}_{3}, 100 \mathrm{MHz}\right)$ spectra of compound 10c
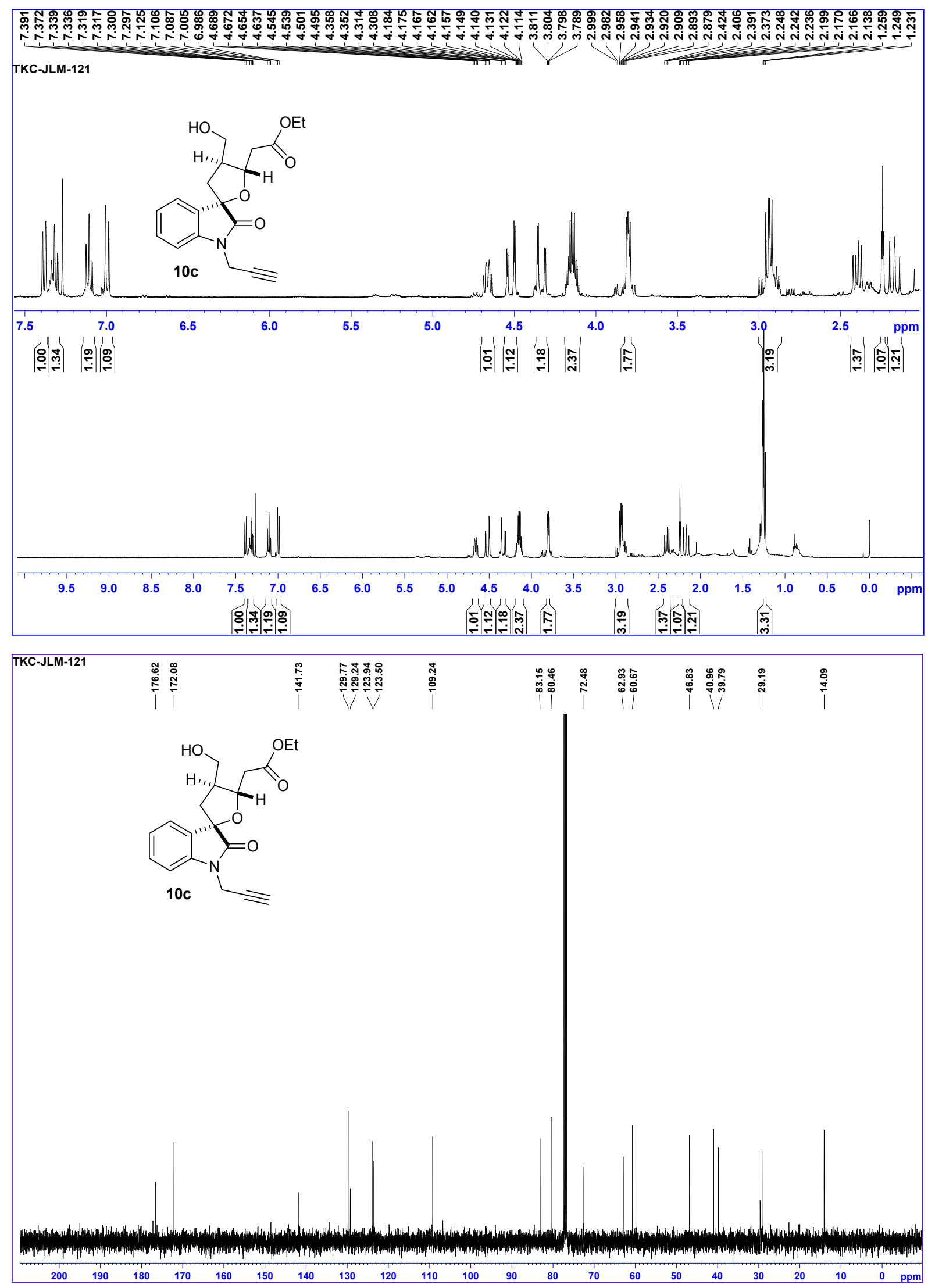
${ }^{1} \mathrm{H}-\mathrm{NMR}\left(\mathrm{CDCl}_{3}, 400 \mathrm{MHz}\right)$ and ${ }^{13} \mathrm{C}-\mathrm{NMR}\left(\mathrm{CDCl}_{3}, 100 \mathrm{MHz}\right)$ spectra of compound $\mathbf{1 0 d}$
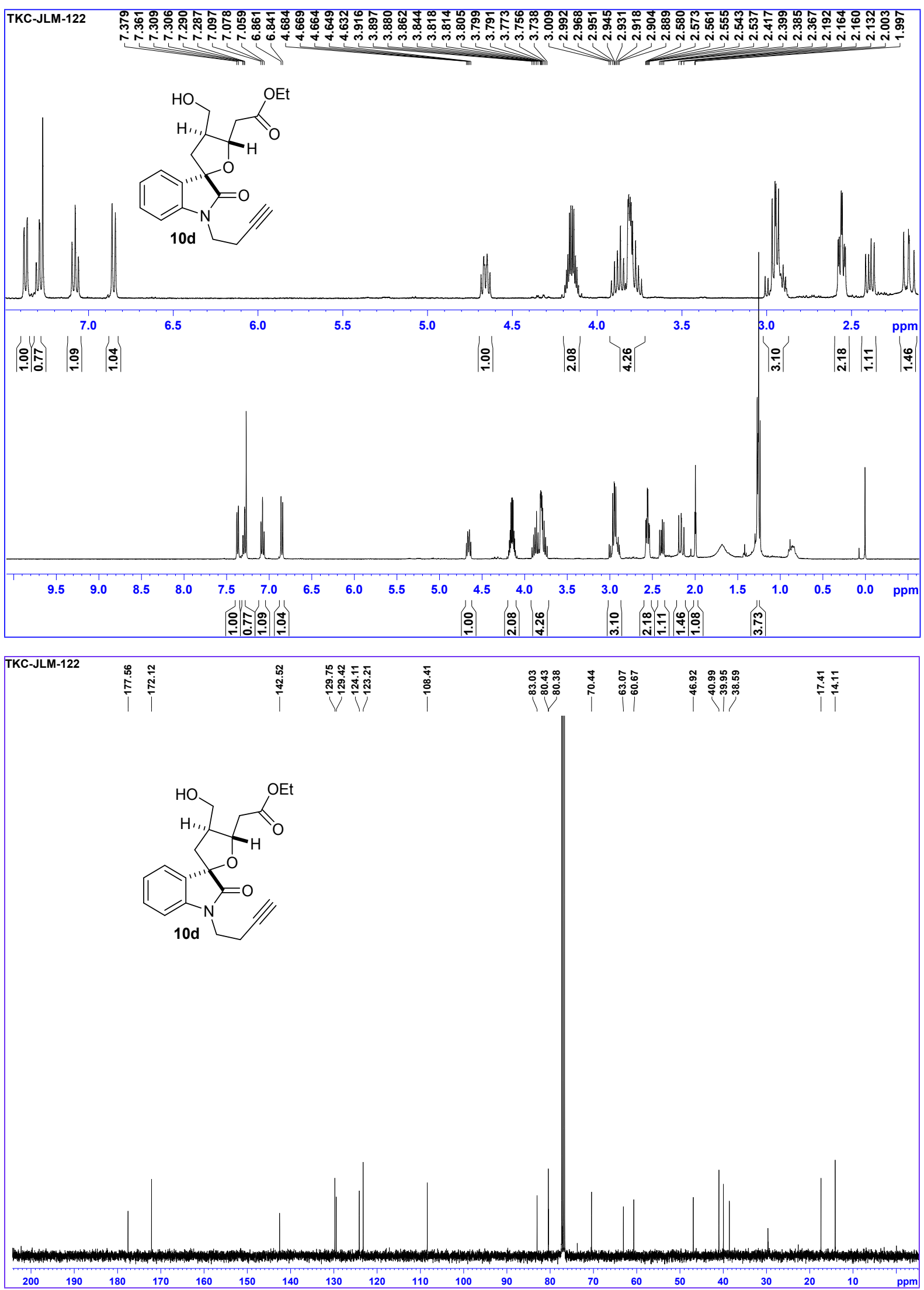
${ }^{1} \mathrm{H}-\mathrm{NMR}\left(\mathrm{CDCl}_{3}, 400 \mathrm{MHz}\right)$ and ${ }^{13} \mathrm{C}-\mathrm{NMR}\left(\mathrm{CDCl}_{3}, 100 \mathrm{MHz}\right)$ spectra of compound 11
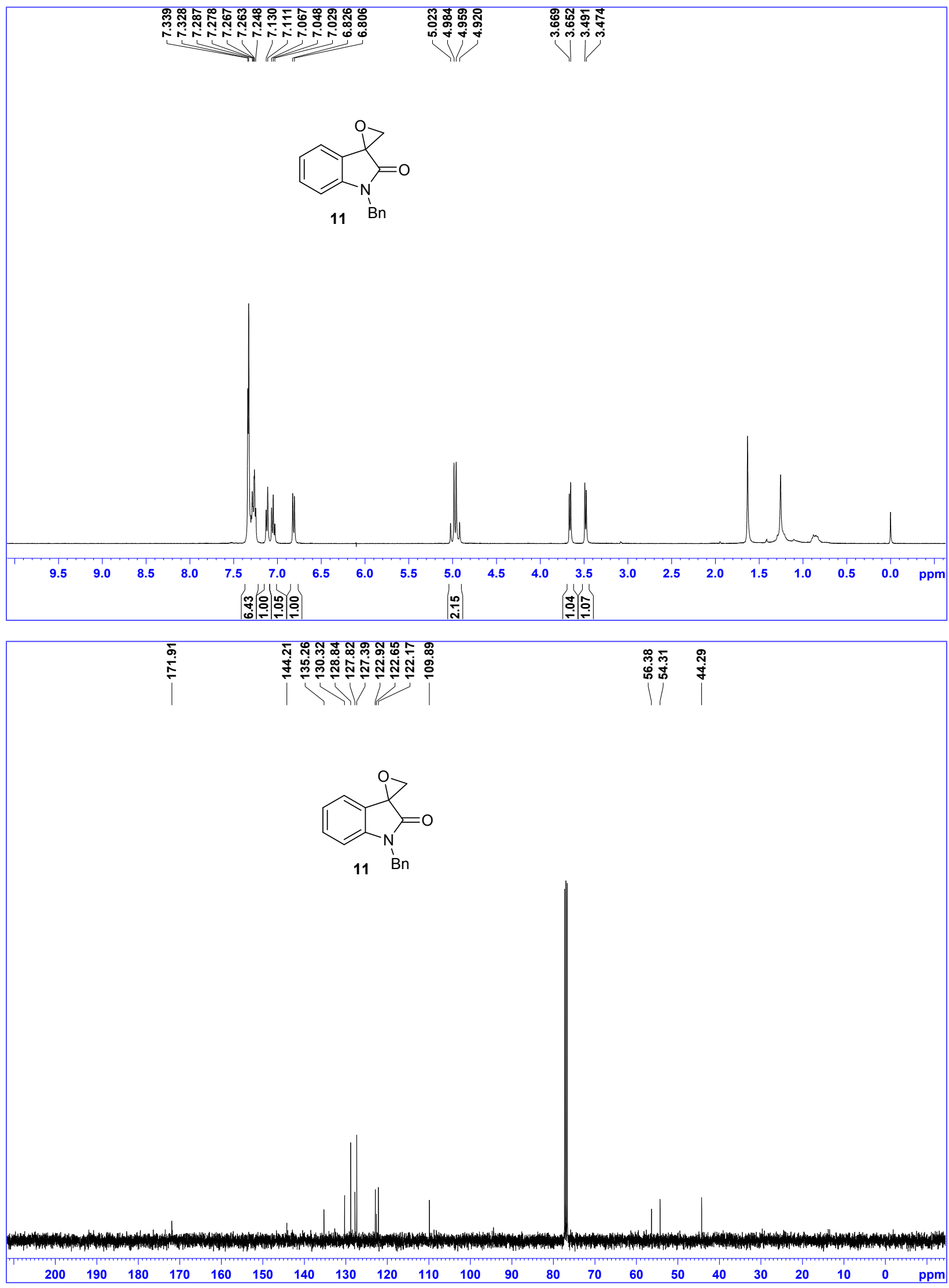
${ }^{1} \mathrm{H}-\mathrm{NMR}\left(\mathrm{CDCl}_{3}, 400 \mathrm{MHz}\right)$ and ${ }^{13} \mathrm{C}-\mathrm{NMR}\left(\mathrm{CDCl}_{3}, 100 \mathrm{MHz}\right)$ spectra of compound 14

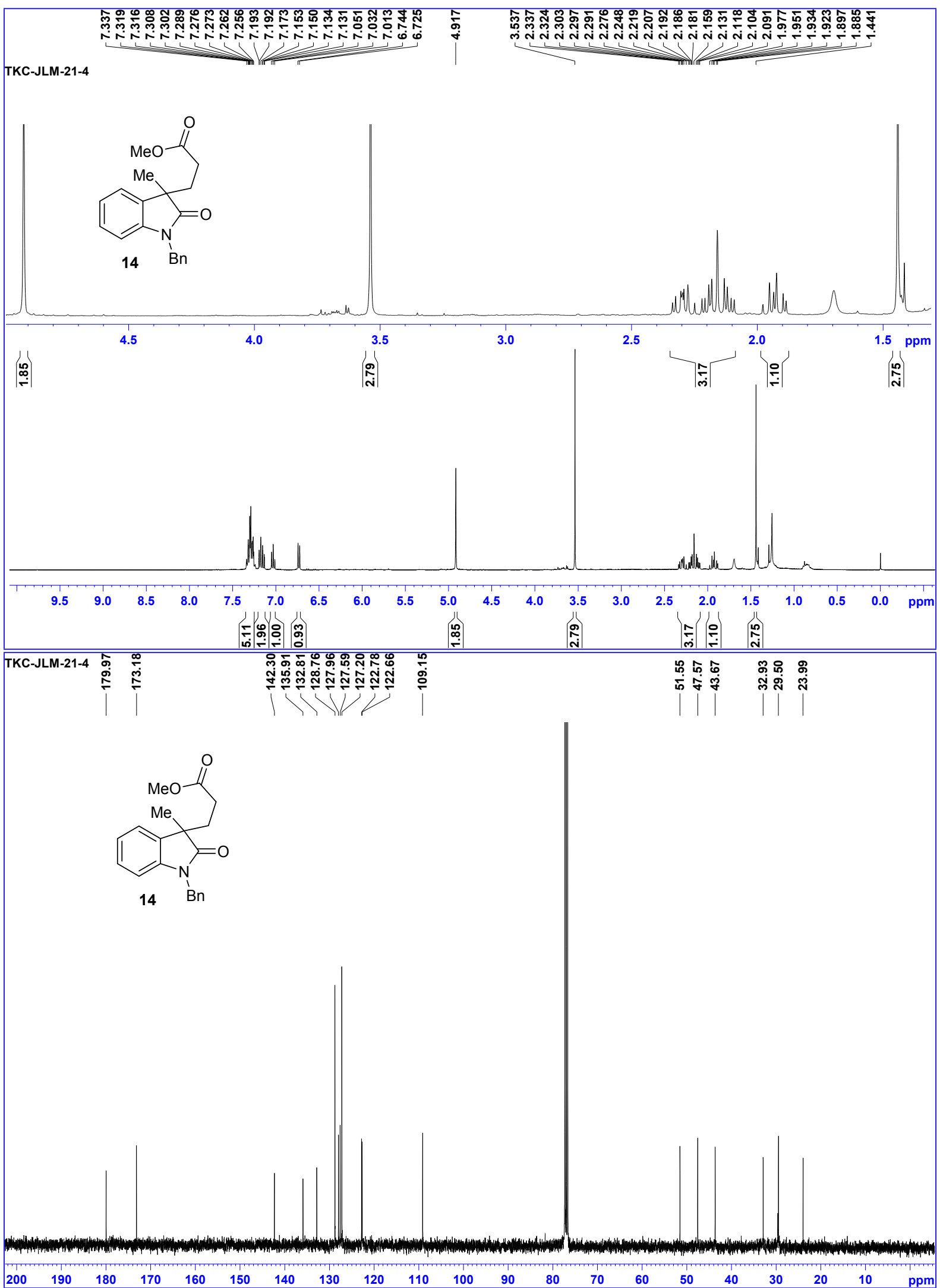


DEPT $\left(\mathrm{CDCl}_{3}, 400 \mathrm{MHz}\right)$ spectra of compound $\mathbf{1 4}$
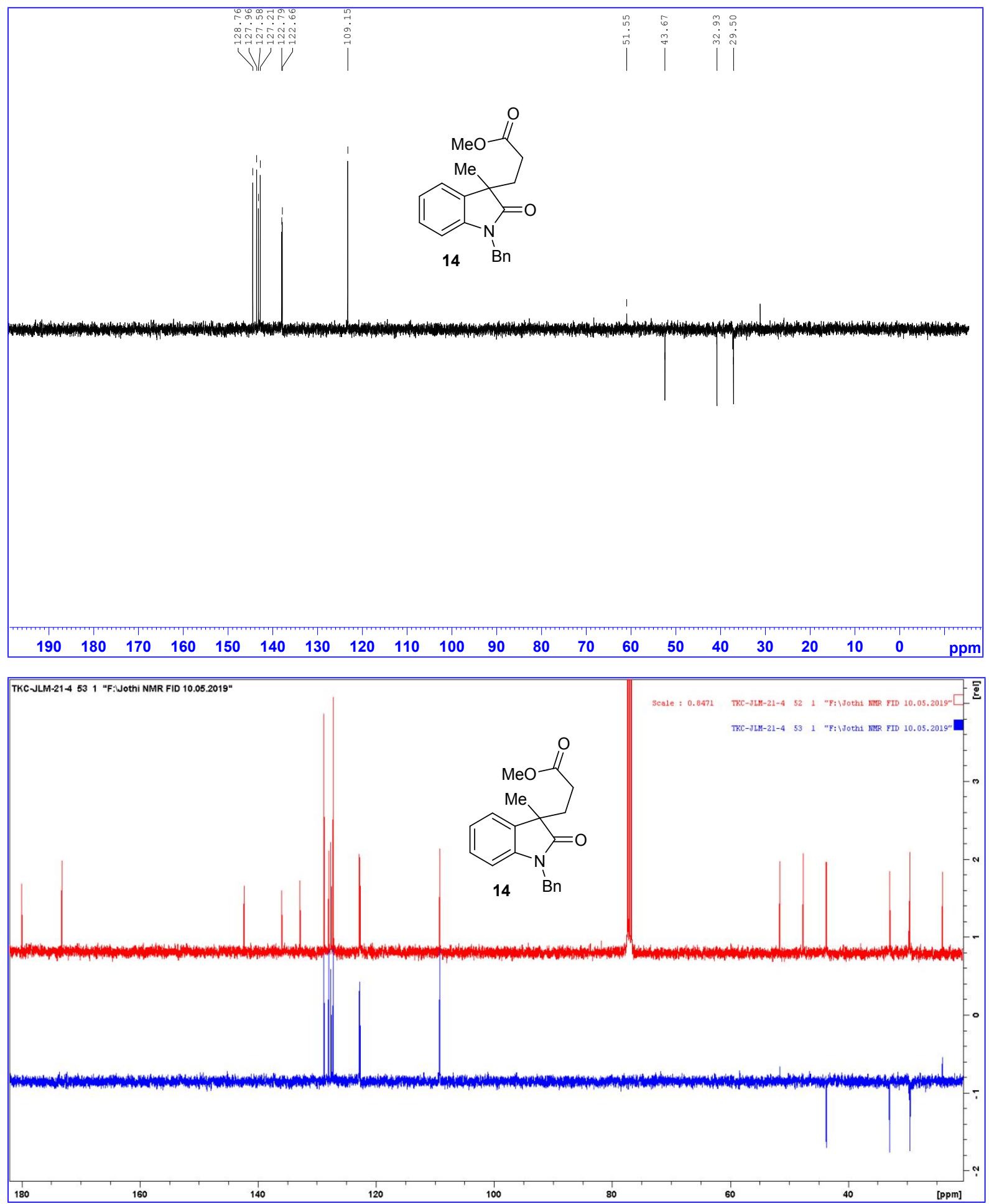
${ }^{1} \mathrm{H}-\mathrm{NMR}\left(\mathrm{CDCl}_{3}, 400 \mathrm{MHz}\right)$ and ${ }^{13} \mathrm{C}-\mathrm{NMR}\left(\mathrm{CDCl}_{3}, 100 \mathrm{MHz}\right)$ spectra of compound 15
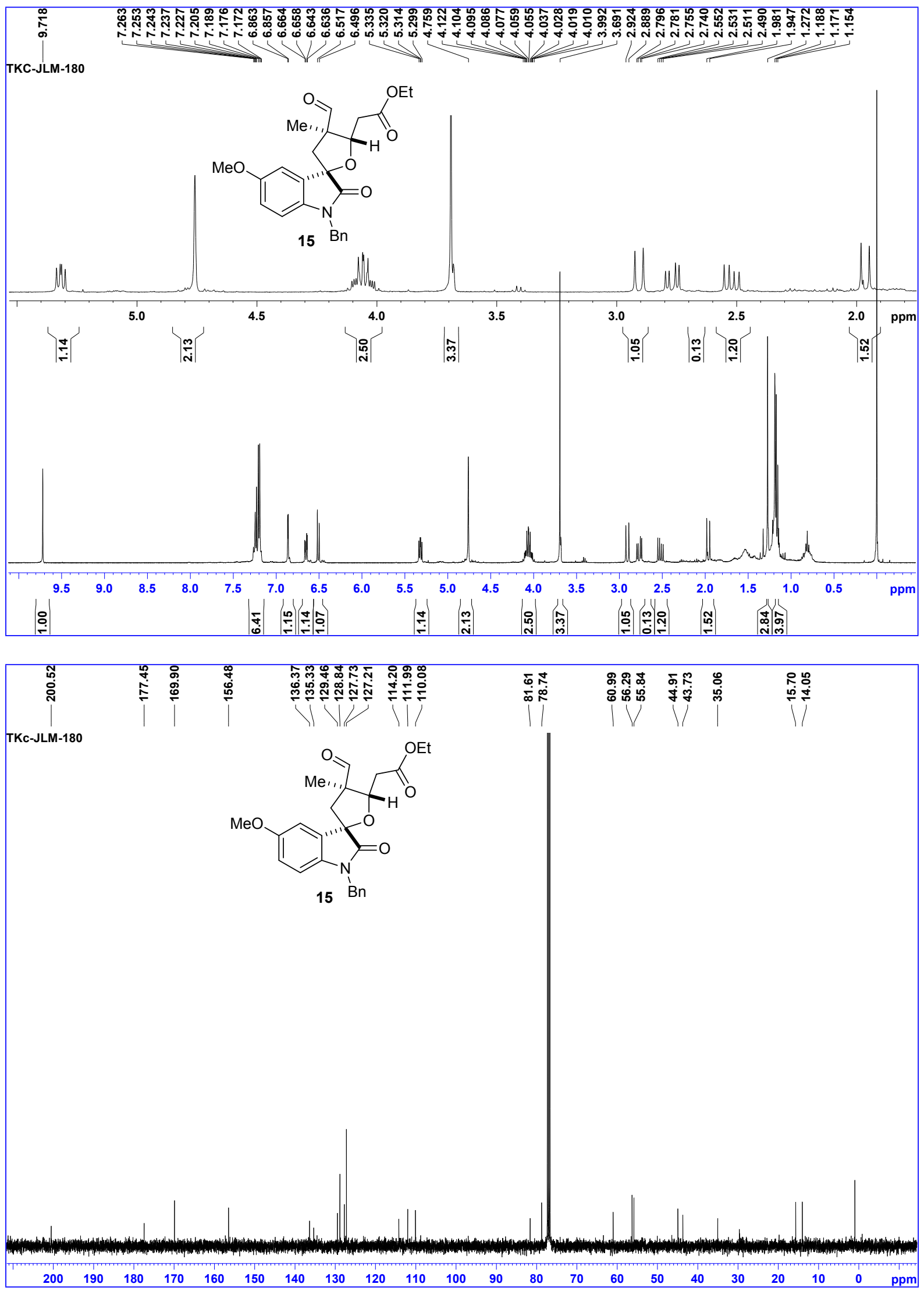
${ }^{1} \mathrm{H}-\mathrm{NMR}\left(\mathrm{CD}_{3} \mathrm{OD}, 400 \mathrm{MHz}\right)$ and ${ }^{13} \mathrm{C}-\mathrm{NMR}\left(\mathrm{CD}_{3} \mathrm{OD}, 100 \mathrm{MHz}\right)$ spectra of compound 16
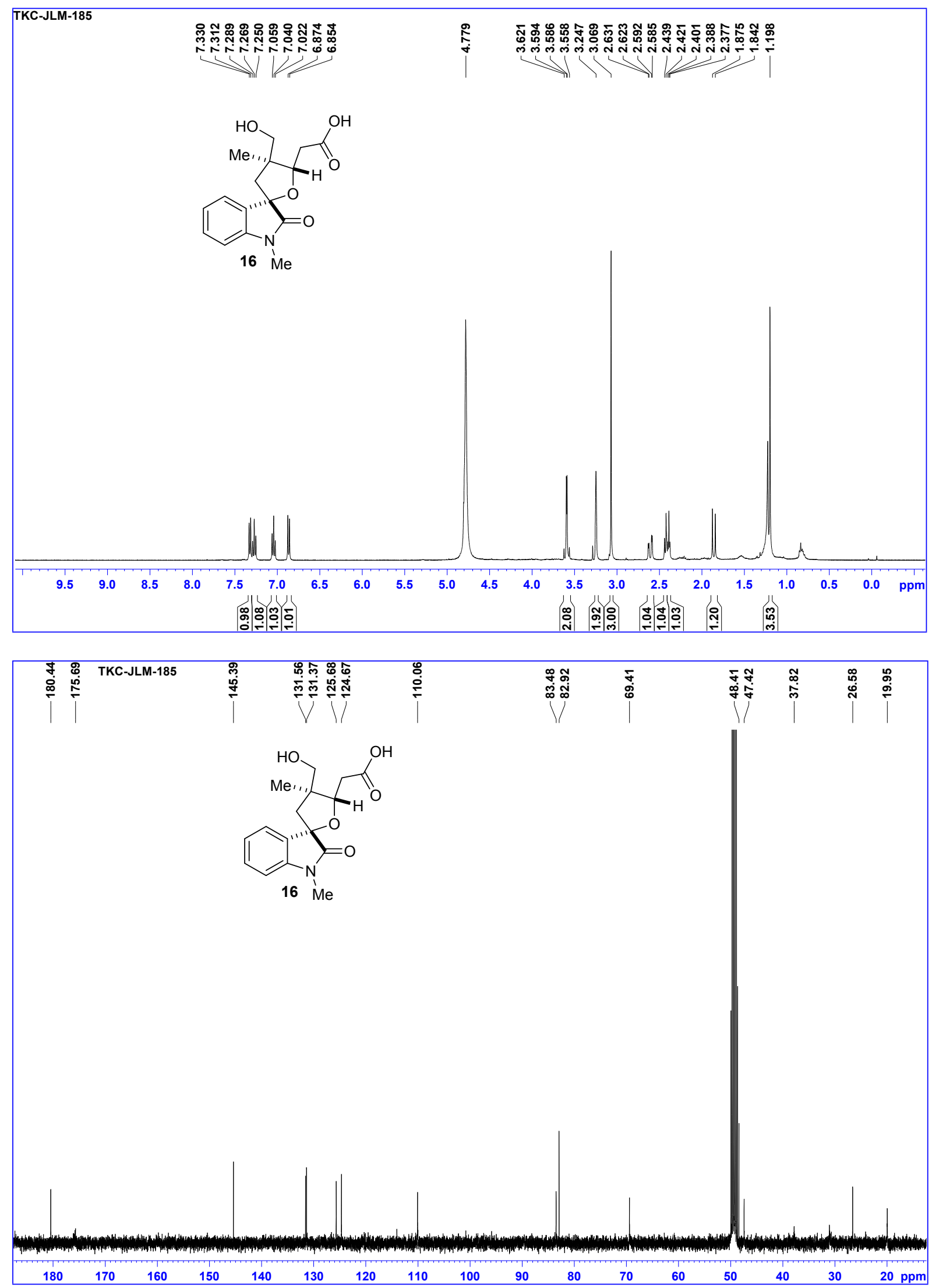
${ }^{1} \mathrm{H}-\mathrm{NMR}\left(\mathrm{CDCl}_{3}, 400 \mathrm{MHz}\right)$ and ${ }^{13} \mathrm{C}-\mathrm{NMR}\left(\mathrm{CDCl}_{3}, 100 \mathrm{MHz}\right)$ spectra of compound 18
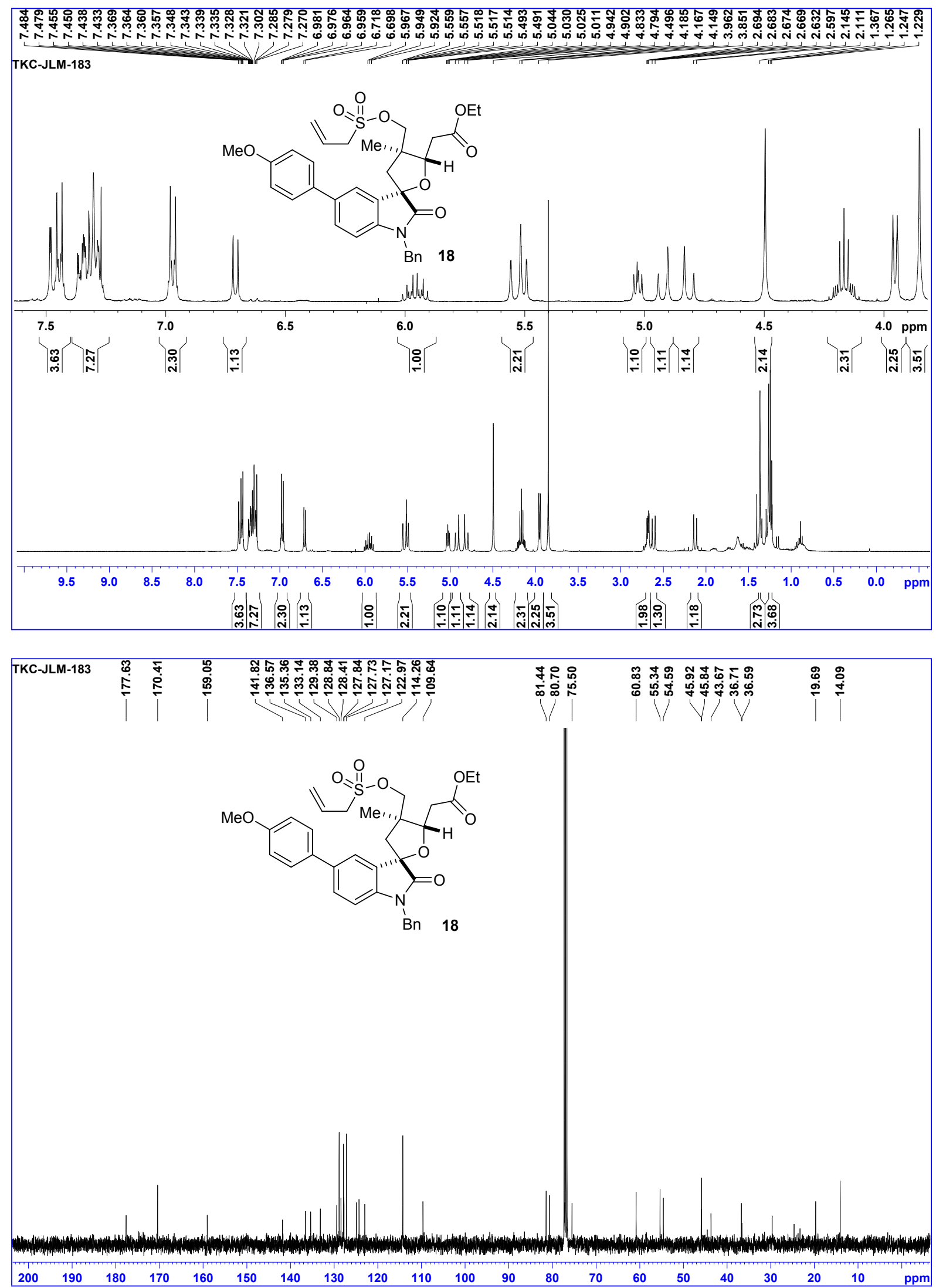
${ }^{1} \mathrm{H}-\mathrm{NMR}\left(\mathrm{CDCl}_{3}, 400 \mathrm{MHz}\right)$ and ${ }^{13} \mathrm{C}-\mathrm{NMR}\left(\mathrm{CDCl}_{3}, 100 \mathrm{MHz}\right)$ spectra of compound 19
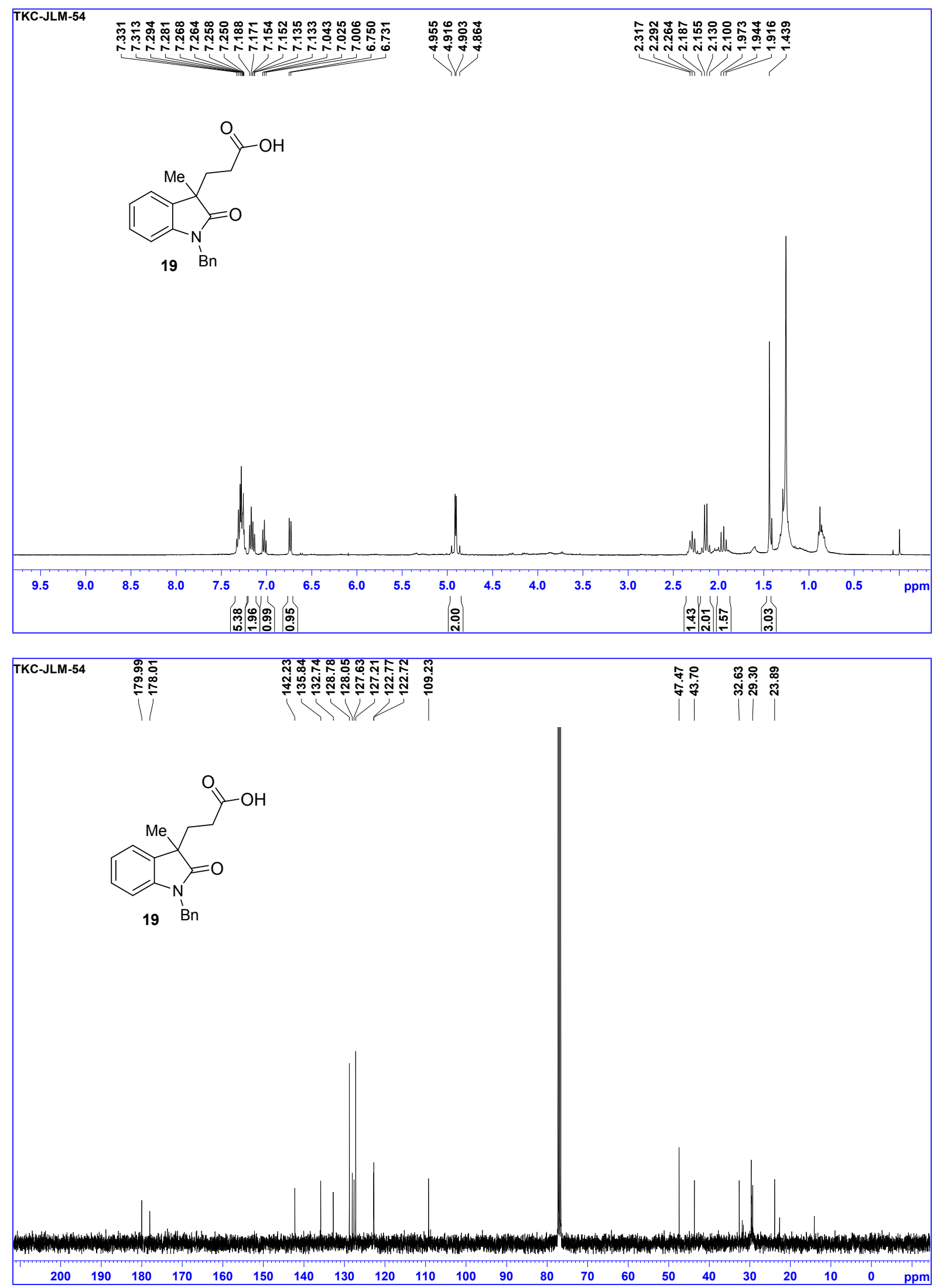
Crystal data and structure refinement for $\mathbf{5 a}$

Identification code

Empirical formula

Formula weight

Temperature

Wavelength

Crystal system

Space group

Unit cell dimensions

Volume

Z

Density (calculated)

Absorption coefficient

$\mathrm{F}(000)$

Crystal size

Theta range for data collection

Index ranges

Reflections collected

Independent reflections

Completeness to theta $=25.242^{\circ}$

Absorption correction

Max. and min. transmission

Refinement method

Data / restraints / parameters

Goodness-of-fit on $\mathrm{F}^{2}$

Final $\mathrm{R}$ indices [I $>2 \operatorname{sigma}(\mathrm{I})]$

$\mathrm{R}$ indices (all data)

Extinction coefficient

Largest diff. peak and hole shelx

$\mathrm{C}_{24} \mathrm{H}_{27} \mathrm{NO}_{5}$

409.46

296(2) K

$0.71073 \AA$

Monoclinic

P 21/n

$\mathrm{a}=14.2282(14) \AA \quad \mathrm{a}=90^{\circ}$.

$\mathrm{b}=9.0871(9) \AA \quad \mathrm{b}=94.998(3)^{\circ}$

$\mathrm{c}=16.8947(16) \AA \quad \mathrm{g}=90^{\circ}$

2176.1(4) $\AA^{3}$

4

$1.250 \mathrm{Mg} / \mathrm{m}^{3}$

$0.087 \mathrm{~mm}^{-1}$

872

$0.429 \times 0.165 \times 0.065 \mathrm{~mm}^{3}$

2.873 to $27.803^{\circ}$.

$-18<=\mathrm{h}<=18,-11<=\mathrm{k}<=11,-22<=\mathrm{l}<=22$

46902

$5120[\mathrm{R}(\mathrm{int})=0.1524]$

$99.9 \%$

Semi-empirical from equivalents

0.7214 and 0.6516

Full-matrix least-squares on $\mathrm{F}^{2}$

$5120 / 0 / 277$

1.010

$\mathrm{R} 1=0.0801, \mathrm{wR} 2=0.1603$

$\mathrm{R} 1=0.1987, \mathrm{wR} 2=0.2108$

$\mathrm{n} / \mathrm{a}$

0.423 and -0.288 e. $\AA^{-3}$
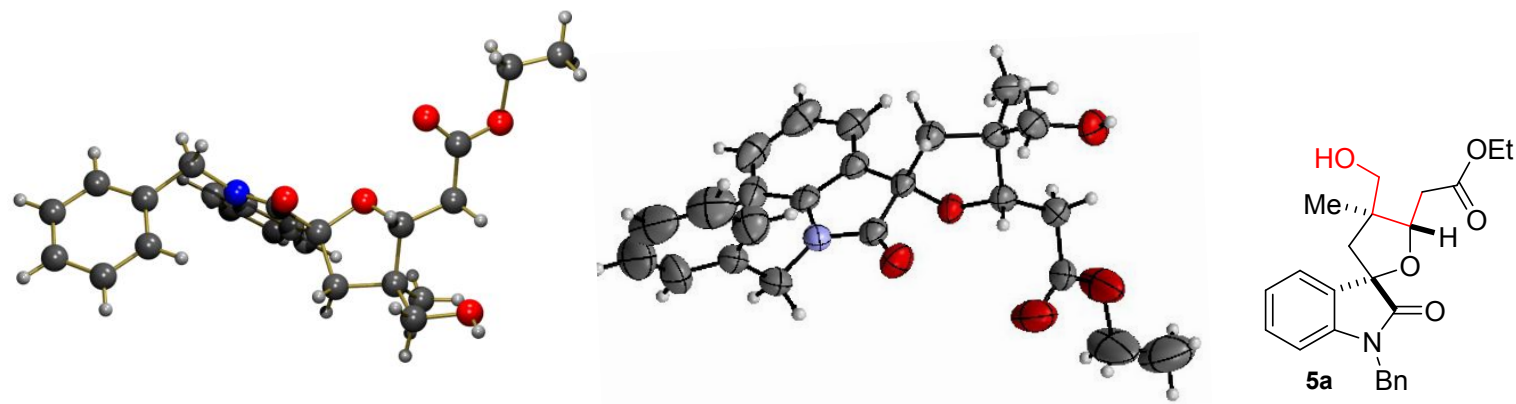

S79 
Crystal structure 5a (thermal ellipsoid at 50\% probability)

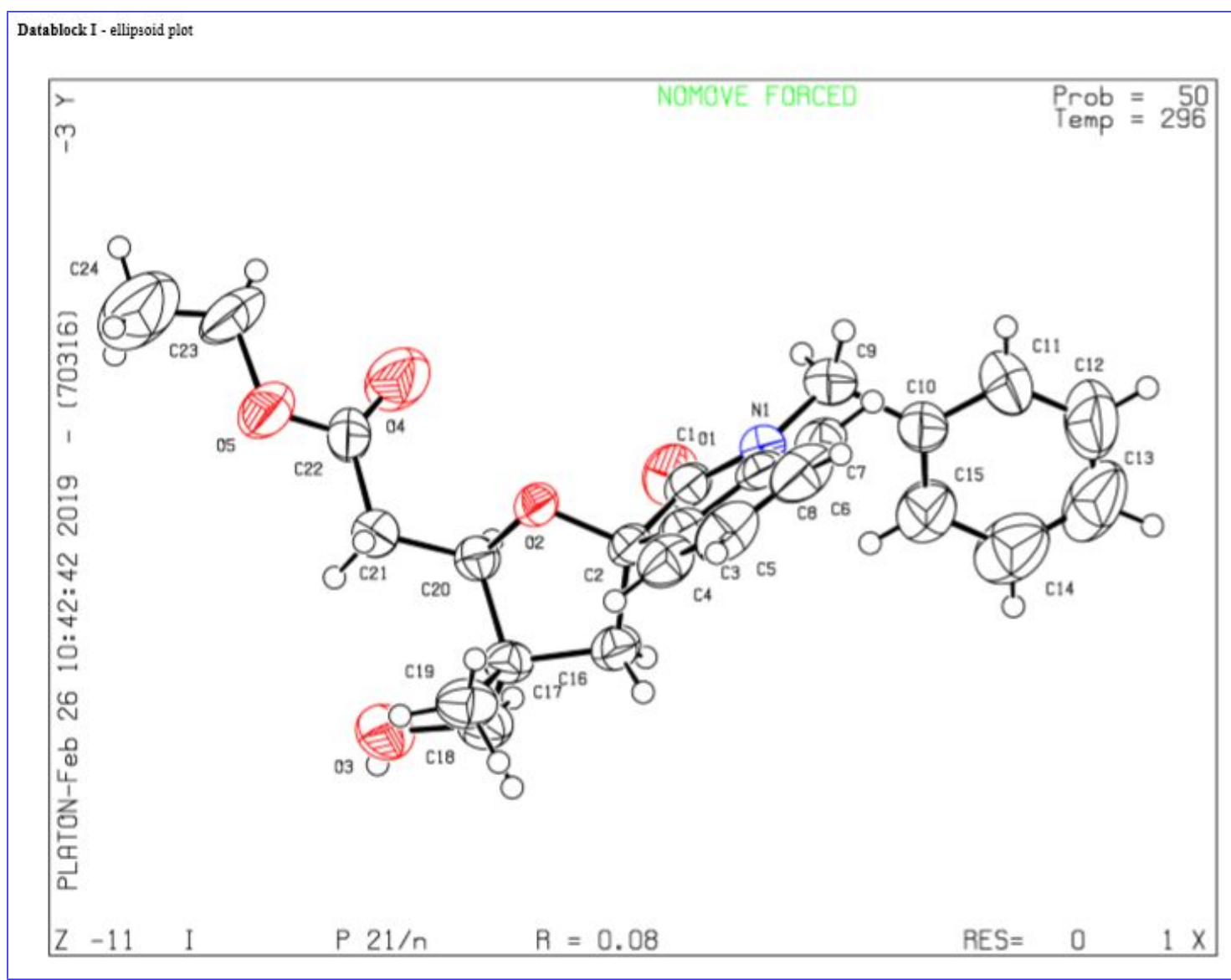


Crystal data and structure refinement for $\mathbf{6 c}$

Identification code

Empirical formula

Formula weight

Temperature

Wavelength

Crystal system

Space group

Unit cell dimensions

Volume

Z

Density (calculated)

Absorption coefficient

$\mathrm{F}(000)$

Crystal size

Theta range for data collection

Index ranges

Reflections collected

Independent reflections

Completeness to theta $=25.242^{\circ}$

Absorption correction

Max. and min. transmission

Refinement method

Data / restraints / parameters

Goodness-of-fit on $\mathrm{F}^{2}$

Final $\mathrm{R}$ indices [I $>2 \operatorname{sigma}(\mathrm{I})]$

$\mathrm{R}$ indices (all data)

Absolute structure parameter

Extinction coefficient

Largest diff. peak and hole shelx

$\mathrm{C}_{18} \mathrm{H}_{19} \mathrm{NO}_{4}$

313.34

296(2) K

$0.71073 \AA$

Monoclinic

P 21

$\mathrm{a}=5.757(4) \AA$

$$
\mathrm{a}=90^{\circ}
$$

$\mathrm{b}=12.135(8) \AA$

$b=102.075(7)^{\circ}$

$\mathrm{c}=11.539(7) \AA$

$\mathrm{g}=90^{\circ}$

788.3(9) $\AA^{3}$

2

\section{$1.320 \mathrm{Mg} / \mathrm{m}^{3}$}

$0.093 \mathrm{~mm}^{-1}$

332

$0.210 \times 0.113 \times 0.097 \mathrm{~mm}^{3}$

3.611 to $30.560^{\circ}$.

$-8<=\mathrm{h}<=8,-17<=\mathrm{k}<=17,-16<=\mathrm{l}<=16$

33881

$4830[\mathrm{R}(\mathrm{int})=0.0210]$

$99.8 \%$

Semi-empirical from equivalents

0.7461 and 0.7050

Full-matrix least-squares on $\mathrm{F}^{2}$

4830 / 1 / 209

1.039

$\mathrm{R} 1=0.0347, \mathrm{wR} 2=0.0960$

$\mathrm{R} 1=0.0378, \mathrm{wR} 2=0.0990$

$-0.19(14)$

$\mathrm{n} / \mathrm{a}$

0.188 and -0.142 e. $\AA^{-3}$
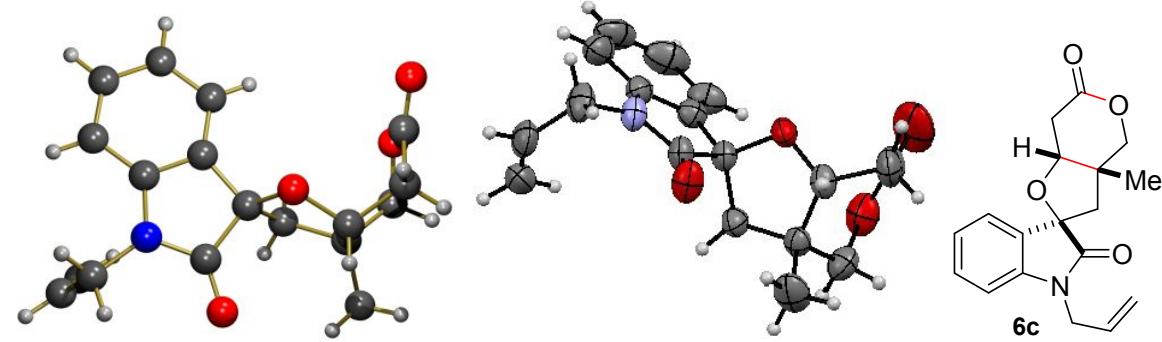
Crystal structure 6c (thermal ellipsoid at $50 \%$ probability)

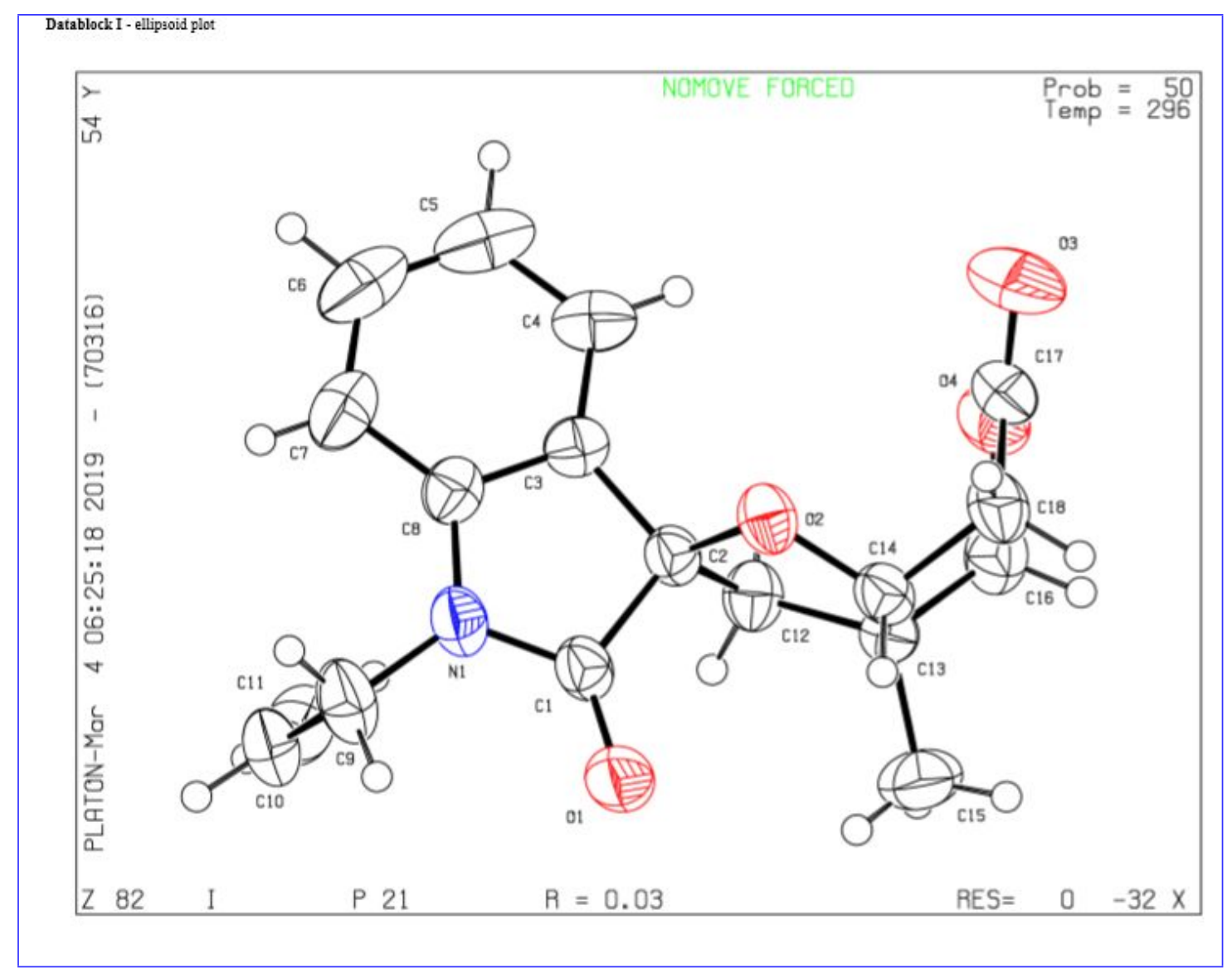

